\title{
Renormalização de aplicações unimodais com ordem crítica próxima a $2 \mathbb{N}$
}

\author{
Judith Hayde Cruz Torres
}

Orientador: Prof. Dr. Daniel Smania Brandão

Tese apresentada ao Instituto de Ciências Matemáticas e de Computação - ICMC-USP, como parte dos requisitos para obtenção do título de Doutor em Ciências - Matemática.

"VERSÃO REVISADA APÓS A DEFESA"

Data da Defesa:

$12 / 11 / 2007$

Visto do Orientador:

USP - São Carlos

Novembro/2007 
Renormalização de aplicações unimodais com ordem crítica próxima a $2 \mathbb{N}$ 
À meus pais, Elsa e Filiberto com muito carinho.

E aos meus irmãos Manuel, Marisol, Gilbert, María. 



\section{Agradecimentos}

Agradeço à Deus por ter sido meu apoio e luz em todo momento ao longo desta caminhada. Aos meus pais e irmãos que estiveram sempre ao meu lado me incentivando. Sem seu apoio, confiança e amor não seria possível alcançar as minhas conquistas.

Aos meus sobrinhos pelos seus carinhos e alegrias que dão aos meus dias, mesmo à distancia.

Ao Professor Dr. Daniel Smania que esteve sempre me orientando. Muito obrigada pela confiança e incentivo recebidos durante toda a minha estada em São Carlos, pelos valiosos ensinamentos, dedicação, acompanhamento e amizade.

Aos professores do ICMC por todos seus ensinamentos. Sou grata a vocês pela dedicação com nós seus alunos.

Às funcionárias do ICMC, pela amizade e atenção dispensada.

Aos meus amigos e colegas do ICMC-USP, com vocês compartilhei muitos momentos de estudo, descontração, alegrias que são inesquecíveis. Obrigada Thiago pela ajuda com o uso do latex, é surpreendente como você sempre tem uma resposta para problemas com o uso deste programa. Em especial, quero agradecer ao Ronny e aos meus amigos Ruth, Walter, Vanessa, Begoña, Elíris, Gloria e Carlos que sempre estiveram presentes, alguns deles na distância, para me ouvir e dar conselhos, não só isso mas também sempre estiveram me alentando e presenteando-me com sua agradável companhia.

Finalmente, agradeço à CAPES pelo apoio financeiro para realização deste trabalho. 



\section{Resumo}

Nós estudamos a dinâmica do operador de renormalização atuando no espaço de pares $(\phi, t)$, onde $\phi$ é um difeomorfismo e $t \in[0,1]$, interpretados como aplicações unimodais $\phi \circ q_{t}$, onde $q_{t}(x)=-2 t|x|^{\alpha}+2 t-1$. Estabelecemos cotas complexas a priori para pares suficientemente renormalizáveis com combinatória limitada e então a utilizamos para mostrar que quando o expoente crítico $\alpha$ está próximo de um número par, o operador de renormalização tem um único ponto fixo, o qual é hiperbólico e possui uma variedade estável de codimensão um que contém todos os pares infinitamente renormalizáveis. 



\begin{abstract}
We study the dynamics of the renormalization operator acting on the space of pairs $(\phi, t)$, where $\phi$ is a diffeomorphism and $t \in[0,1]$, interpretated as unimodal maps $\phi \circ q_{t}$, where $q_{t}(x)=-2 t|x|^{\alpha}+2 t-1$. We prove the so called complex bounds for sufficiently renormalizable pairs with bounded combinatorics. This allows us to show that if the critical exponent $\alpha$ is close to an even number then the renormalization operator has a unique fixed point. Furthermore this fixed point is hyperbolic and its codimension one stable manifold contains all infinitely renormalizable pairs.
\end{abstract}





\section{Sumário}

Introdução $\quad 3$

1 Renormalização Unimodal 9

1.1 Noções usuais da renormalização unimodal . . . . . . . . . . . . . . . . . . 9

1.2 Decomposições . . . . . . . . . . . . . . . . . . . . 14

1.3 Aplicações univalentes . . . . . . . . . . . . . . . . . . 15

1.4 Cotas reais e complexas a priori . . . . . . . . . . . . . . 20

2 Operador Composição 33

2.1 Espaços de Banach e seus espaços tangentes. . . . . . . . . . . . . . . . 34

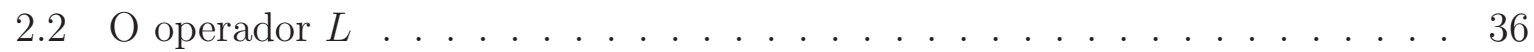

2.3 O diferencial de $L$ e suas propriedades . . . . . . . . . . . . . . . . . . . . 38

3 Operadores de Renormalização Complexos 41

3.1 Operador complexo $\mathcal{R}_{\alpha} \ldots \ldots \ldots \ldots \ldots$. . . . . . . . . . . . 41

3.2 Operador complexo $\widetilde{\mathcal{R}}_{\alpha} \ldots \ldots \ldots \ldots \ldots \ldots \ldots$

3.3 Relação entre os operadores complexos $\mathcal{R}_{2 n}$ e $\widetilde{\mathcal{R}}_{2 n} \ldots \ldots$. . . . . . . . . 48

4 Hiperbolicidade para $\alpha \sim 2 n \quad 53$

4.1 Hiperbolicidade do ponto fixo do operador $\widetilde{\mathcal{R}}_{2 n} \ldots \ldots \ldots$. . . . . . 53

4.2 Hiperbolicidade para $\alpha \sim 2 n \ldots \ldots \ldots \ldots \ldots$ 
A Operadores compactos $\quad 65$

A.1 Espectro de um operador compacto . . . . . . . . . . . . 65

$\begin{array}{ll}\text { B Resultados de análise complexa } & 67\end{array}$

B.1 Aplicacões holomorfas . . . . . . . . . . . . . . . . . 67

B.2 Aplicações quaseconformes . . . . . . . . . . . . . . . . . . 68

B.3 Aplicações do tipo polinomial . . . . . . . . . . . . . . . . . . . . . 69 


\section{Introdução}

Investigando o fenômeno da universalidade de propriedades geométricas de certos sistemas dinâmicos, Feigenbaum e P. Coullet \& C. Tresser independentemente definiram o operador de renormalização de duplicação de período sobre um espaço apropriado de aplicações unimodais. Importantes contribuições na teoria de renormalização foram motivadas pela conjectura de que o operador de duplicação de período, atuando no espaço de aplicações unimodais, tem um único ponto fixo, o qual é hiperbólico com direção instável uni-dimensional.

O primeiro passo para provar a existência de um ponto fixo para o operador de duplicação de período foi devido a Lanford em 1981. Ele mostrou a existência de ponto fixo para o operador de renormalização com expoente crítico $\alpha=2$ por meio de uma prova assistida por computador. Sullivan [6] estabeleceu a existência dos conjuntos invariantes do tipo ferradura para o operador renormalização com expoente crítico par, $\alpha=2 n$, onde $n \in \mathbb{N}$, e que estas ferraduras atraem todas as transformações unimodais infinitamente renormalizáveis com combinatória limitada, fazendo uso da dinâmica complexa das aplicações do tipo polinomial no sentido de Douady e Hubbard. Sua combinação de análise real e complexa abriu novas direcões na teoria dos sistemas dinâmicos. Posteriormente C. McMullen [3] provou a convergência exponencial da renormalização com combinatória limitada. Finalmente Lyubich [16] provou a conjectura de Feigenbaum-Coullet-Tresser afirmando que o ponto fixo do operador de duplicação de período é hiperbólico, com variedade estável de codimensão um.

Primeiramente faremos uma introdução rápida da definição do operador renormalização $\mathcal{R}$. Fixemos $\alpha>1$, o chamado expoente crítico. A família unimodal "padrão" 
$q_{t}:[-1,1] \rightarrow[-1,1]$, com $t \in[0,1]$ e expoente crítico $\alpha$, é definida por

$$
q_{t}(x)=-2 t|x|^{\alpha}+2 t-1 .
$$

Seja $\operatorname{Diff}_{+}^{1}([-1,1])$ o conjunto de difeomorfismos do intervalo $[-1,1]$, que preservam orientação. Consideremos

$$
\mathcal{U}=\operatorname{Diff}_{+}^{1}([-1,1]) \times[0,1],
$$

onde um elemento $(\phi, t) \in \mathcal{U}$ será interpretado como uma aplicação unimodal

$$
f=\phi \circ q_{t}:[-1,1] \rightarrow[-1,1],
$$

com expoente crítico $\alpha>1$. A aplicação unimodal $f=\phi \circ q_{t}$ é dita renormalizável se existe um ponto periódico repulsor $p \in(-1,1)$, tal que a aplicação de primeiro retorno para o intervalo $I_{q}=[-|p|,|p|]$ é da forma $f^{q}: I_{q} \rightarrow I_{q} \operatorname{com} f^{q}(p)=p$ e $q \geq 2$. A aplicação de primeiro retorno para $I_{q}$ será, depois de um re-escalonamento, uma aplicação unimodal definida no intervalo $[-1,1]$. O período da renormalização é o menor inteiro $q$ satisfazendo as propriedades acima mencionadas. Assim definimos o operador de renormalização $\mathcal{R}$ tal que para a aplicação unimodal renormalizável $f=\phi \circ q_{t}$ temos que $\mathcal{R} f$ é uma aplicação unimodal definida como

$$
\mathcal{R} f(z)=\frac{1}{p} f^{q}(p z)
$$

$z \in[-1,1]$. A aplicação unimodal $\mathcal{R} f$ é chamada de renormalização de $f$.

Existem pelo menos duas dificuldades no estudo do operador renormalização $\mathcal{R}$ quando $\alpha$ não é um número par:

- Não podemos fazer uso de métodos da dinâmica complexa para estudar este operador.

- O operador $\mathcal{R}$ não é diferenciável.

Tendo em vista esses problemas, neste trabalho definimos um novo operador de renormalização $\widetilde{\mathcal{R}}$ num espaço apropriado de pares $(\phi, t)$, onde $\phi \circ q_{t}$ é uma aplicação unimodal. Um par $(\phi, t) \in \mathcal{U}$ é renormalizável com ordem crítica $\alpha$ se e somente se a aplicação unimodal $f=\phi \circ q_{t}$ tem um ciclo, isto é uma coleção $\mathcal{I}=\left\{I_{1}, I_{2}, \ldots, I_{q}\right\}$ de intervalos fechados em $[-1,1]$ satisfazendo: 
1. existe um ponto periódico repulsor $p \in(-1,1)$ com $I_{q}=[-|p|,|p|]$.

2. $f: I_{i} \rightarrow I_{i+1}, i=1,2, \ldots, q-1$, é aplicação monótona e sobrejetiva,

3. $f\left(I_{q}\right) \subset I_{1} \operatorname{com} f(p) \in \partial I_{1}$, o bordo de $I_{1}$,

4. os interiores de $I_{1}, I_{2}, \ldots, I_{q}$ são dois a dois disjuntos.

Os aspectos combinatoriais de uma renormalização são descritos por permutações unimodais. Dotando um ciclo $\mathcal{I}=\left\{I_{1}, I_{2}, \ldots, I_{q}\right\}$ com a relação de ordem $\prec$ definida por: $I_{j} \prec I_{i}, j \neq i$ se e somente se $\inf I_{j}<\inf I_{i}$. A aplicação $\sigma: \mathcal{I} \rightarrow \mathcal{I}$ tal que

$$
\sigma\left(I_{i}\right)=I_{i+1} \bmod q
$$

é uma permutação. Uma permutação sobre o conjunto ordenado $\mathcal{I}$ é permutação unimodal se esta satisfaz a seguinte condição. Mergulhando $\mathcal{I}$ na reta real preservando a ordem $\prec$, o gráfico da permutação $\sigma$ sobre $\mathbb{R}^{2}$ pode ser estendido, pela união dos pontos consecutivos do gráfico de $\sigma$ por segmentos de retas, para o gráfico que corresponde a uma aplicação unimodal.

Seja $\sigma$ uma permutação unimodal e

$$
\mathcal{U}_{\sigma}=\left\{(\phi, t) \in \mathcal{U} \mid f=\phi \circ q_{t} \text { tem um ciclo } \mathcal{I} \operatorname{com} \sigma(\mathcal{I})=\sigma\right\}
$$

Seja $I \subset[-1,1]$ um intervalo orientado e consideremos o operador zoom

$$
Z_{I}: \operatorname{Diff}_{+}^{1}([-1,1]) \rightarrow \operatorname{Diff}_{+}^{1}([-1,1])
$$

o qual faz corresponder ao difeomorfismo $\phi: I \rightarrow \phi(I)$ o difeomorfismo $Z_{I}(\phi)$, obtido através da normalização do domínio $I$ e imagem $\phi(I)$ para o intervalo $[-1,1]$ via transformações afins. Os intervalos $I$ e $\phi(I)$ tem a mesma orientação. Seja $(\phi, t) \in \mathcal{U}_{\sigma}$. Definimos o difeomorfismo que preserva orientação $\phi_{0}:[-1,1] \rightarrow[-1,1]$, dado por

$$
\phi_{0}=Z_{\phi^{-1}\left(I_{1}\right)}(\phi)
$$


Aqui $I_{0}=I_{q}$. Por outro lado para cada $I_{i} \in \mathcal{I}, i \neq 0$, definimos os difeomorfismos, que preservam orientação $q_{i}:[-1,1] \rightarrow[-1,1]$ e $\phi_{i}:[-1,1] \rightarrow[-1,1]$ dados por

$$
q_{i}=Z_{I_{i}}\left(q_{t}\right) \text { e } \phi_{i}=Z_{q_{t}\left(I_{i}\right)}(\phi)
$$

donde $q_{t}\left(I_{i}\right)$ e $I_{i+1}$ são orientados na mesma direção, quer dizer a orientação $o\left(I_{i+1}\right)$ definida pelo ciclo $\mathcal{I}$. Além disso, seja

$$
t_{1}=\frac{\left|q_{t}\left(I_{q}\right)\right|}{\left|\phi^{-1}\left(I_{1}\right)\right|}
$$

Assim podemos definir o novo operador renormalização

$$
\widetilde{\mathcal{R}}_{\sigma}: U_{\sigma} \rightarrow U
$$

por

$$
\widetilde{\mathcal{R}}_{\sigma}(\phi, t)=\left(\left(\phi_{q-1} \circ q_{q_{-1}}\right) \circ \cdots \circ\left(\phi_{2} \circ q_{2}\right) \circ\left(\phi_{1} \circ q_{1}\right) \circ \phi_{0}, t_{1}\right) .
$$

Observe que para cada $\sigma$ existe uma única fatorização maximal $\sigma=<\sigma_{n}, \ldots, \sigma_{2}, \sigma_{1}>$ tal que

$$
\widetilde{\mathcal{R}}_{\sigma}=\widetilde{\mathcal{R}}_{\sigma_{n}} \circ \cdots \circ \widetilde{\mathcal{R}}_{\sigma_{2}} \circ \widetilde{\mathcal{R}}_{\sigma_{1}}
$$

Uma permutação unimodal $\sigma$ é chamada de prima se e somente se $\sigma=\langle\sigma\rangle$. Fazendo uso das permutações unimodais primas obtemos uma partição do conjunto de pares renormalizáveis em $\mathcal{U}$ e assim o operador de renormalização pode ser definido como

$$
\widetilde{\mathcal{R}}_{\alpha}:\{\text { pares renormalizáveis }\}=\bigcup_{\sigma \text { primo }} \mathcal{U}_{\sigma} \rightarrow \mathcal{U},
$$

donde $\widetilde{\mathcal{R}}_{\alpha} \mid \mathcal{U}_{\sigma}=\widetilde{\mathcal{R}}_{\sigma}$.

Marco Martens [17] provou a existência de pontos fixos do operador de renormalização $\mathcal{R}_{\alpha}$, de qualquer tipo combinatorial $\sigma$, agindo no espaço de aplicações unimodais suaves com expoente crítico $\alpha>1$. Para este fim ele introduziu o chamado método de decomposição.

Uma das principais ferramentas no desenvolvimento do presente trabalho são as chamadas "cotas complexas a priori" (complex bounds): esta consiste em achar um domínio 
complexo $V \supset[-1,1]$ tal que para $n$ suficientemente grande a primeira componente de

$$
\widetilde{\mathcal{R}}(\phi, t)=\left(\left(\phi_{q_{n}-1}^{n} \circ q_{q_{n}-1}^{n}\right) \circ \ldots \circ\left(\phi_{2}^{n} \circ q_{2}^{n}\right) \circ\left(\phi_{1}^{n} \circ q_{1}^{n}\right) \circ \phi_{0}^{n}, t_{n}\right)
$$

é bem definida e univalente em $V$. Aqui obtemos cotas complexas estudando a decomposição de difeomorfismos da primeira componente do novo operador renormalização. Nos fazemos uso de cotas reais (real bounds) para obter controle sobre a geometria dos ciclos de aplicações $N$-vezes renormalizáveis, para $N$ suficientemente grande, de combinatória limitada por uma constante $B>0$, e também fazemos uso de algumas ferramentas de análise complexa. Como consequência deste resultado temos que para cada par unimodal infinitamente renormalizável $(\phi, t)$ com combinatória limitada por $B>0$, a sequência de aplicações formadas pelas primeiras coordenadas dos pares $\widetilde{\mathcal{R}}_{\alpha}^{n}(\phi, t), n \geq 1$, forma uma família pré-compacta de aplicações sobre qualquer sub-domínio compacto de $V$ (veja Corolário 1.20).

A vantagem de lidar com o novo operador renormalização é que, devido à propriedade de cotas complexas, $\widetilde{\mathcal{R}}_{\alpha}$ é um operador analítico complexo compacto e $\alpha \mapsto \widetilde{\mathcal{R}}_{\alpha}$ é uma família de operadores $C^{\omega}$ (veja a Proposição 3.4). Tudo isto nos permite usar métodos perturbativos para concluir que para $\alpha$ próximo a um número par existe um ponto fixo hiperbólico para $\widetilde{\mathcal{R}}_{\alpha}$.

Para $\alpha=2 n$, usaremos os resultados obtidos por Martens, McMullen, Lyubich and E. de Faria, W. de Melo and Pinto para mostrar que o operador $\widetilde{\mathcal{R}}_{2 n}$ tem um único ponto fixo o qual é hiperbólico. Isto é obtido introduzindo (Capítulo 2) o operador analítico complexo chamado o operador composição

$$
L:(\phi, t) \rightarrow \phi \circ q_{t}
$$

O operador composição $L$ relaciona a dinâmica do operador renormalização $\mathcal{R}_{2 n}$ com o novo operador de renormalização $\widetilde{\mathcal{R}}_{2 n}$. De fato

$$
\mathcal{R}_{2 n} \circ L=L \circ \widetilde{\mathcal{R}}_{2 n}
$$

O operador $L$ não é inversível, porém veremos que ele possui várias propriedades: injetividade, compacidade, e sua derivada $\mathcal{D} L$ tem imagem densa. Tais propriedades nos 
permitirão concluir que o espectro do ponto fixo $\left(\phi^{*}, t^{*}\right)$ de $\widetilde{\mathcal{R}}_{2 n}$ coincide como o espectro do ponto fixo $\phi^{*} \circ q_{t^{*}}$ de $\mathcal{R}_{2 n}$.

No Capítulo 4 apresentaremos resultados de unicidade, hiperbolicidade e universalidade para pontos fixos do operador de renormalização quando consideramos o expoente crítico $\alpha \sim 2 n$. Edson de Faria, W. de Melo and A. Pinto [8], com a ajuda de cotas reais e complexas a priori de Sullivan [6] e o resultado de hiperbolicidade de Lyubich [16] (veja também [5]), provaram a hiperbolicidade de algum iterado do operador renormalização $\mathcal{R}_{2 n}$ agindo no espaço $\mathcal{U}_{D_{\varepsilon / 2}}$ de aplicações holomorfas $f$ no domínio complexo $D_{\varepsilon / 2}$, e contínuos na fronteira, da forma $f=\psi\left(x^{2}\right)$ numa vizinhança de $[-1,1]$, com $f(-1)=f(1)=-1$. Mais precisamente, o Teorema 2.4 em [8] garante que existe um conjunto aberto $O$, contido no espaço de Banach $\mathcal{U}_{D_{\varepsilon / 2}}$, onde $\varepsilon>0$, e $N$ positivo com a seguinte propriedade: o operador analítico real $\mathcal{R}_{2 n}^{N}: O \rightarrow \mathcal{U}_{D_{\varepsilon / 2}}$ tem ponto fixo hiperbólico $f^{*}=\phi^{*} \circ q_{t^{*}} \in O$ com variedade estável de codimensão um. Em outras palavras a derivada do operador de renormalização $\mathcal{D} \mathcal{R}^{N}\left(\phi^{*} \circ q_{t^{*}}\right)$, um operador linear e compacto, tem espectro com um único autovalor simples fora do disco unitário e os outros autovalores estão no interior do disco unitário. Fazendo uso do operador composição $L$ obtemos a hiperbolicidade do ponto fixo $\left(\phi^{*}, t^{*}\right)$ do operador renormalização $\widetilde{\mathcal{R}}_{2 n}$, com variedade estável de codimensão um.

Na Seção 4.2, para $\alpha$ suficientemente próximo de $2 n$, proporcionamos as seguintes respostas positivas para as questões que motivaram nosso trabalho:

- Unicidade do ponto fixo. O operador renormalização $\widetilde{\mathcal{R}}_{\alpha}$ tem um único ponto fixo unimodal $\left(\phi_{\alpha}^{*}, t_{\alpha}^{*}\right)$ (Teorema 4.8).

- Hiperbolicidade do ponto fixo. O ponto fixo $\left(\phi_{\alpha}^{*}, t_{\alpha}^{*}\right)$ é hiperbólico, com variedade estável de codimensão um. Além disso $\alpha \rightarrow\left(\phi_{\alpha}^{*}, t_{\alpha}^{*}\right)$ é uma função analítica real (Teorema 4.6).

- Universalidade do ponto fixo. Se um par unimodal $(\phi, t)$ é infinitamente renormalizável com ordem crítica $\alpha$ então ele pertence a variedade estável do ponto fixo $\left(\phi_{\alpha}^{*}, t_{\alpha}^{*}\right)$ de $\widetilde{\mathcal{R}}_{\alpha}$ (Teorema 4.15). 


\section{Capítulo 1}

\section{Renormalização Unimodal}

Nas Seções 1.1 e 1.2 apresentamos noções usuais dos operadores de renormalização $\mathcal{R}$ e $\widetilde{\mathcal{R}}$. Nós seguimos as definições e notações para o operador $\mathcal{R}$ de A. Avila, M. Martens e W. de Melo [1]. Usamos a decomposição de M. Martens [17] para definir o novo operador de renormalização $\widetilde{\mathcal{R}}$. Na ultima seção apresentamos a cota real a priori para obter controle sobre a geometria dos ciclos dos pares $N$-vezes renormalizáveis, para $N$ suficientemente grande, com combinatória limitada por uma constante $B>0$. Também usamos as ferramentas de análise complexa da Seção 1.3 para apresentar o primeiro dos resultados importantes deste trabalho, as cotas complexas a priori.

\subsection{Noções usuais da renormalização unimodal}

Fixemos $\alpha>1$, o expoente crítico. A família unimodal "padrão" $q_{t}:[-1,1] \rightarrow[-1,1]$, com $t \in[0,1]$ e expoente $\alpha$, é definida por

$$
q_{t}(x)=-2 t|x|^{\alpha}+2 t-1
$$

O parâmetro $t$ determina o máximo $q_{t}(0)=2 t-1$. Seja Diff ${ }_{+}^{1}([-1,1])$ o conjunto de difeomorfismos $\mathrm{C}^{1}$ que preservam orientação do intervalo $[-1,1]$. Este é um subconjunto 
aberto do espaço de Banach $\mathrm{C}^{1}([-1,1], \mathbb{R})$ das aplicações $\mathrm{C}^{1}$ dotadas com a norma do supremo

$$
|f|=\sup _{x \in-1,1]}\{|f(x)|,|D f(x)|\}
$$

Considere

$$
\mathcal{U}=\operatorname{Diff}_{+}^{1}([-1,1]) \times[0,1],
$$

onde um elemento $(\phi, t) \in \mathcal{U}$ devera ser interpretado como a aplicação unimodal

$$
f=\phi \circ q_{t}:[-1,1] \rightarrow[-1,1]
$$

com expoente crítico $\alpha>1$. O difeomorfismo $\phi$ é chamado a parte difeomorfa da aplicação unimodal $f$. A métrica sobre $\mathcal{U}$ é o produto da métrica induzida pela norma do supremo sobre $\operatorname{Diff}_{+}^{1}([-1,1])$ e a métrica do intervalo.

Definição 1.1 Uma coleção $\mathcal{I}=\left\{I_{1}, I_{2}, \ldots, I_{q}\right\}$ de intervalos fechados em $[-1,1]$ é chamada de ciclo para a aplicação unimodal $f$ se tem as seguintes propriedades

1. Existe um ponto periódico repulsor $p \in(-1,1)$ com $I_{q}=[-|p|,|p|]$.

2. $f: I_{i} \rightarrow I_{i+1}, i=1,2, \ldots, q-1$, é aplicação monótona e sobrejetiva.

3. $f\left(I_{q}\right) \subset I_{1} \operatorname{com} f(p) \in \partial I_{1}$, o bordo de $I_{1}$.

4. Os interiores de $I_{1}, I_{2}, \ldots, I_{q}$ são dois a dois disjuntos.

Definição 1.2 Considere uma coleção $\mathcal{I}=\left\{I_{1}, I_{2}, \ldots, I_{q}\right\}$ de intervalos fechados em $[-1,1]$ o qual é dotado com a relação de ordem $\prec$ definida por:

$$
I_{j} \prec I_{i}, j \neq i \text {, se e somente se } \inf I_{j}<\inf I_{i} \text {. }
$$

Dizemos que uma aplicação $\sigma: \mathcal{I} \rightarrow \mathcal{I}$ tal que

$$
\sigma\left(I_{i}\right)=I_{i+1} \bmod q
$$

é uma permutação. Chamamos de permutação unimodal $\sigma$ com respeito á relação de ordem $\prec$ se esta satisfaz a seguinte condição. Mergulhando $\mathcal{I}$ na reta real preservando a 

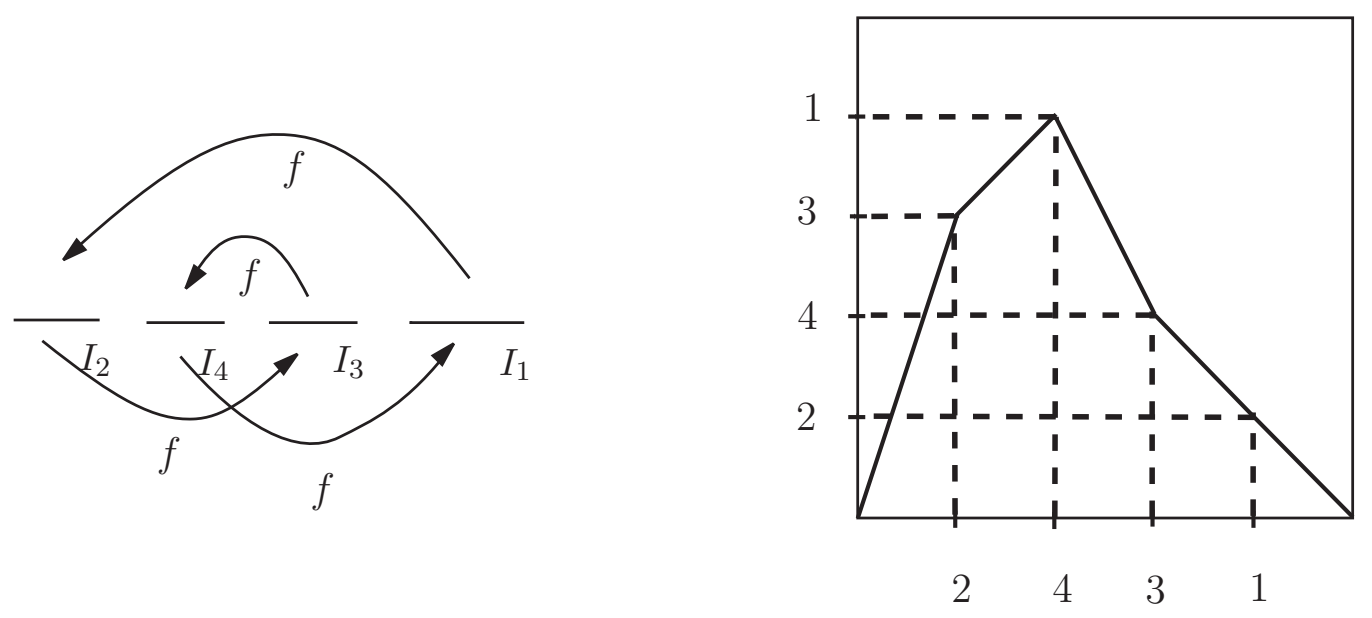

Figura 1.1: Permutação unimodal de quatro elementos.

ordem $\prec$, o gráfico da permutação $\sigma$ sobre $\mathbb{R}^{2}$ pode ser estendido, pela união dos pontos consecutivos do gráfico de $\sigma$ por segmentos de retas, para o gráfico que corresponde a uma aplicação unimodal.

Exemplo 1.3 A figura 1.1 corresponde ao gráfico estendido de uma permutação unimodal $\sigma$ definida sobre o ciclo $\mathcal{I}=\left\{I_{1}, I_{2}, I_{3}, I_{4}\right\}$ de uma aplicação unimodal $f$, onde os intervalos fechados que formam o ciclo estão ordenados como $I_{2} \prec I_{4} \prec I_{3} \prec I_{1}$.

Como conseqüência direta da definição do ciclo $\mathcal{I}=\left\{I_{1}, I_{2}, \ldots, I_{q}\right\}$ temos

- $\mathcal{I}$ herda um ordem de $[-1,1]$,

- a aplicação

$$
\sigma=\sigma(\mathcal{I}): I_{i} \mapsto I_{i+1} \bmod q
$$

sobre $\mathcal{I}$ é uma permutação unimodal,

- a orientação

$$
o_{\mathcal{I}}: \mathcal{I} \rightarrow\{-1,1\}
$$

é definida tal que $o_{\mathcal{I}}\left(I_{i}\right)=1$ quando $f^{i}(p)$ é o extremo esquerdo de $I_{i}$ e $o_{\mathcal{I}}\left(I_{i}\right)=-1$ em outro caso. Assim temos que o ciclo $\mathcal{I}$ é orientado. 
Definição 1.4 Uma aplicação unimodal $f=\phi \circ q_{t}$ é chamada renormalizável se e somente se tem um ciclo. A aplicação de primeiro retorno para $I_{q}$ será, depois de um re-escalamento, uma aplicação unimodal. O período da renormalização é o menor $q$ satisfazendo as propriedades acima. Defina o operador de renormalização $\mathcal{R}$ tal que para uma aplicação unimodal renormalizável $f=\phi \circ q_{t}$ temos que $\mathcal{R} f$ é a aplicação unimodal definida como

$$
\mathcal{R} f(z)=\frac{1}{p} f^{q}(p z)
$$

$z \in[-1,1]$. A aplicação unimodal $\mathcal{R} f$ é chamada de renormalização de $f$.

Definição $1.5 \operatorname{Um}$ par $(\phi, t) \in \mathcal{U}$ é chamado renormalizável se $f=\phi \circ q_{t}$ é renormalizável. O período de renormalização de $(\phi, t)$ é o mesmo de $f$.

Seja $\sigma$ uma permutação unimodal e

$$
\mathcal{U}_{\sigma}=\left\{(\phi, t) \in \mathcal{U} \mid f=\phi \circ q_{t} \text { tem um ciclo } \mathcal{I} \operatorname{com} \sigma(\mathcal{I})=\sigma\right\}
$$

Nos referiremos algumas vezes aos pares em $\mathcal{U}_{\sigma}$ de $\sigma$-renormalizáveis para enfatizar o tipo combinatorial de renormalização em consideração.

Seja $I \subset[-1,1]$ um intervalo orientado e consideremos o operador zoom

$$
Z_{I}: \operatorname{Diff}_{+}^{1}([-1,1]) \rightarrow \operatorname{Diff}_{+}^{1}([-1,1]),
$$

o qual faz corresponder ao difeomorfismo $\phi: I \rightarrow \phi(I)$, o difeomorfismo $Z_{I}(\phi):[-1,1] \rightarrow$ $[-1,1]$ definido por:

$$
Z_{I}(\phi)=A_{\phi(I)} \circ \phi \circ A_{I}^{-1}
$$

onde a transformação $A_{J}: J \rightarrow[-1,1]$ é a única transformação afim, que preserva orientação, que leva o intervalo fechado $J$ para o intervalo $[-1,1]$. Os intervalos $I$ e $\phi(I)$ tem a mesma orientação.

Seja $(\phi, t) \in \mathcal{U}_{\sigma}$. Definimos o difeomorfismo que preserva orientação $\phi_{0}:[-1,1] \rightarrow$ $[-1,1]$ onde

$$
\phi_{0}=Z_{\phi^{-1}\left(I_{1}\right)}(\phi) .
$$


Aqui $I_{0}=I_{q}$. Por outro lado para cada $I_{i} \in \mathcal{I}, i \neq 0$, definimos os difeomorfismos, que preservam orientação, $q_{i}:[-1,1] \rightarrow[-1,1]$ e $\phi_{i}:[-1,1] \rightarrow[-1,1]$ onde

$$
q_{i}=Z_{I_{i}}\left(q_{t}\right)
$$

e

$$
\phi_{i}=Z_{q_{t}\left(I_{i}\right)}(\phi)
$$

onde $q_{t}\left(I_{i}\right)$ e $I_{i+1}$ são orientados na mesma direção, isto é a orientação $o\left(I_{i+1}\right)$ definida pelo ciclo $\mathcal{I}$. Além disso, seja

$$
t_{1}=\frac{\left|q_{t}\left(I_{q}\right)\right|}{\left|\phi^{-1}\left(I_{1}\right)\right|} .
$$

Desde que $f\left(I_{q}\right)=\phi \circ q_{t}\left(I_{q}\right) \subset I_{1}$, na definição do ciclo $\mathcal{I}$, temos que $t_{1} \in[0,1]$. Isto é equivalente a dizer que

$$
q_{t}(0) \in \phi^{-1}\left(I_{1}\right)
$$

Agora podemos passar a definir o operador de renormalização.

Definição 1.6 O operador de $\sigma$-renormalização

$$
\widetilde{\mathcal{R}}_{\sigma}: U_{\sigma} \rightarrow U
$$

é definido por

$$
\widetilde{\mathcal{R}}_{\sigma}(\phi, t)=\left(\left(\phi_{q-1} \circ q_{q_{-1}}\right) \circ \ldots \circ\left(\phi_{2} \circ q_{2}\right) \circ\left(\phi_{1} \circ q_{1}\right) \circ \phi_{0}, t_{1}\right)
$$

O operador de $\sigma$-renormalização $\widetilde{\mathcal{R}}_{\sigma}$ é definido dando lugar ao ciclo $\mathcal{I} \operatorname{com} \sigma(\mathcal{I})=\sigma$. Para cada $\sigma$ existe uma única fatorização maximal $\sigma=<\sigma_{n}, \ldots, \sigma_{2}, \sigma_{1}>$ tal que

$$
\widetilde{\mathcal{R}}_{\sigma}=\widetilde{\mathcal{R}}_{\sigma_{n}} \circ \ldots \circ \widetilde{\mathcal{R}}_{\sigma_{2}} \circ \widetilde{\mathcal{R}}_{\sigma_{1}}
$$

Uma permutação unimodal $\sigma$ é chamada prima se e somente se $\sigma=<\sigma>$. Obviamente cada permutação na fatorização maximal é prima. Assim fazendo uso das permutações unimodais primas obtemos uma partição do conjunto de pares renormalizáveis em $\mathcal{U}$. 
Definição 1.7 O operador de renormalização denotado por

$$
\widetilde{\mathcal{R}}:\{\text { pares renormalizáveis }\}=\bigcup_{\sigma \text { primo }} \mathcal{U}_{\sigma} \rightarrow \mathcal{U},
$$

é definido como $\widetilde{\mathcal{R}} \mid \mathcal{U}_{\sigma}=\widetilde{\mathcal{R}}_{\sigma}$.

Definição $1.8 U m$ par $(\phi, t) \in \mathcal{U}$ é $N$-vezes renormalizável se e somente se $\widetilde{\mathcal{R}}^{n}(\phi, t)$ é definido para todo $1 \leq n \leq N$.

Fixemos um conjunto finito de permutações primas $\Sigma=\left\{\sigma_{1}, . ., \sigma_{n}\right\}$.

Definição 1.9 Um par $N$-vezes renormalizável $(\phi, t) \in \mathcal{U}$, para $N$ suficientemente grande, tem combinatória limitada por $B>0$ se a aplicação unimodal $f=\phi \circ q_{t}$ tem períodos de renormalização tal que $q_{n+1} / q_{n} \leq B$, para todo $1 \leq n<N$.

\subsection{Decomposições}

Nesta seção seguimos basicamente parte da exposição e notação da Seção 2 em [1]. Aqui observamos que a sequência de pares em $\mathcal{U}$, produzida aplicando repetidamente o operador de renormalização $\widetilde{\mathcal{R}}_{\alpha}$, é tal que cada um dos pares tem como primeira componente uma decomposição de difeomorfismos e a segunda componente um parâmetro que leva a informação da parte unimodal "padrão" da aplicação unimodal.

Fixemos um par $f=(\phi, t) \in \mathcal{U} N$-vezes renormalizável e seja $\mathcal{I}^{n}=\left\{I_{1}^{n}, I_{2}^{n}, \ldots, I_{q_{n}}^{n}\right\}$ o ciclo correspondente à $n$-ésima renormalização, $1 \leq n \leq N$. Cada ciclo será particionado em conjuntos

$$
\mathcal{I}^{n}=\bigcup_{k \geq 0}^{N} L_{k}^{n}
$$

Os conjuntos de nível $L_{k}^{n}, k \geq 0$, são definidos por indução. Seja

$$
\mathcal{I}^{0}=L_{0}^{0}=\{[-1,1]\}
$$


Se $\mathcal{I}^{n+1} \ni I_{i}^{n+1} \subset I_{j}^{n} \in L_{k}^{n}$ e $0 \notin I_{i}^{n+1}$ então $I_{i}^{n+1} \in L_{k+1}^{n+1}$. Se $0 \in I_{i}^{n+1}$ então $I_{i}^{n+1} \in L_{0}^{n+1}$. Observe que

$$
\mathcal{I}^{n}=\bigcup_{k \geq 0}^{n} L_{k}^{n},
$$

para $n \leq N$. Primeiro para $I_{1}^{n} \in \mathcal{I}^{n}$, defina o difeomorfismo, que preserva orientação, $\phi_{0}^{n}:[-1,1] \rightarrow[-1,1]$ onde

$$
\phi_{0}^{n}=Z_{\phi^{-1}\left(I_{1}^{n}\right)}(\phi) .
$$

Então para cada $I_{i}^{n} \in \mathcal{I}^{n}, i \neq 0$, defina os difeomorfismos, que preservam orientação, $q_{i}^{n}:[-1,1] \rightarrow[-1,1]$ e $\phi_{i}^{n}:[-1,1] \rightarrow[-1,1]$ onde

$$
q_{i}^{n}=Z_{I_{i}^{n}}\left(q_{t}\right)
$$

$\mathrm{e}$

$$
\phi_{i}^{n}=Z_{q_{t}\left(I_{i}^{n}\right)}(\phi)
$$

onde $q_{t}\left(I_{i}^{n}\right)$ e $I_{i+1}^{n}$ são orientados na mesma direção, isto é com a orientação $o\left(I_{i+1}^{n}\right)$ definida pelo ciclo $\mathcal{I}^{n}$. Além disso, seja

$$
t_{n}=\frac{\left|q_{t}\left(I_{0}^{n}\right)\right|}{\left|\phi^{-1}\left(I_{1}^{n}\right)\right|}
$$

As definições acima descrevem a primeira componente de $\widetilde{\mathcal{R}}^{n}(\phi, t)$ que consiste da decomposição dos difeomorfismos $q_{i}^{n}$ e $\phi_{i}^{n}$ :

$$
\widetilde{\mathcal{R}}^{n}(\phi, t)=\left(\left(\phi_{q_{n}-1}^{n} \circ q_{q_{n}-1}^{n}\right) \circ \ldots \circ\left(\phi_{2}^{n} \circ q_{2}^{n}\right) \circ\left(\phi_{1}^{n} \circ q_{1}^{n}\right) \circ \phi_{0}^{n}, t_{n}\right) .
$$

\subsection{Aplicações univalentes}

Nesta seção apresentamos alguns resultados sobre a classe de funções univalentes sobre o disco unitário $\mathbb{D}=\{z \in \mathbb{C}:|z|<1\}$. Alguns destes resultados são apresentados sem prova em vista que são bem conhecidos de análise complexa e podem ser encontrados nas referências dadas. Lembremos que um subconjunto não vazio $U \subset \mathbb{C}$ é chamado um domínio se ele é um conjunto aberto e conexo no plano complexo. E uma região é um subconjunto não vazio aberto no plano complexo. Em vista de que cada região 
simplesmente conexa propriamente contida no plano complexo é a imagem de $\mathbb{D}$ por uma equivalência conforme, o estudo de funções univalentes sobre $\mathbb{D}$ é equivalente ao estudo de funções univalentes sobre regiões simplesmente conexas arbitrarias.

Os resultados apresentados nesta seção vão nos dar ferramentas que nos permitirão obter "cota complexa a priori" o qual é explicado de maneira detalhada na próxima seção.

Definição 1.10 Uma função analítica $\varphi$ é univalente num dominio $U$ se é injetiva.

Definição 1.11 A classe $\mathcal{S}$ consiste de todas as funções univalentes $\varphi$ sobre $\mathbb{D}$ tal que $\varphi(0)=0$ e $\varphi^{\prime}(0)=1$

Seguimos com o Teorema de Distorção de Koebe (1907) o qual proporciona cotas para a derivada de funções univalentes.

Teorema $1.12([13])$ Se $\varphi \in \mathcal{S}$, então

$$
\frac{1-r}{(1+r)^{3}} \leq\left|\varphi^{\prime}(z)\right| \leq \frac{1+r}{(1-r)^{3}} \text { se }|z| \leq r
$$

Corolário $1.13([9])$ Se $\varphi \in \mathcal{S}$, então

$$
\left|\arg \varphi^{\prime}(z)\right| \leq 2 \log \left(\frac{1+r}{1-r}\right) \text { para }|z| \leq r .
$$

Em vista destes resultados podemos estabelecer o seguinte Teorema.

Teorema 1.14 Para qualquer $K>1$ e $1<\epsilon<K / 2$ o seguinte se satisfaz: se $\phi e ́$ uma aplicação univalente definida na bola $B(0, K)$ satisfazendo $\phi(-1)=-1, \phi(1)=1$ e $\phi([-1,1]) \subset[-1,1]$, então

$$
|\phi-\mathrm{id}|_{B(0, \epsilon)}<O\left(\frac{\epsilon}{K}\right) .
$$

Demonstração. Considere a função

$$
\varphi(z)=\frac{\phi(K z)-\phi(0)}{K \phi^{\prime}(0)} .
$$


Esta função pertence à classe $\mathcal{S}$. De acordo com o Teorema 1.12

$$
\left|\varphi^{\prime}(z)\right| \leq \frac{1+|z|}{(1-|z|)^{3}}
$$

para $|z|<1$. Fazendo as substituições apropriadas obtemos

$$
\frac{1-|z|}{(1+|z|)^{3}} \leq\left|\frac{\phi^{\prime}(K z)}{\phi^{\prime}(0)}\right| \leq \frac{1+|z|}{(1-|z|)^{3}}
$$

Para $|w| \leq \epsilon$ tome $z=w / K$ assim $|z| \ll 1$ e temos que

$$
\left|\frac{\phi^{\prime}(w)}{\phi^{\prime}(0)}\right| \geq \frac{1-\frac{\epsilon}{K}}{\left(1+\frac{\epsilon}{K}\right)^{3}}=1-O(\epsilon / K)
$$

e

$$
\left|\frac{\phi^{\prime}(w)}{\phi^{\prime}(0)}\right| \leq \frac{1+\frac{\epsilon}{K}}{\left(1-\frac{\epsilon}{K}\right)^{3}}=1+O(\epsilon / K)
$$

Existe $z_{0} \in[-1,1] \subset B(0, \epsilon)$ tal que $\phi^{\prime}\left(z_{0}\right)=1$. Escolhendo $w=z_{0}$ obtemos

$$
1-O(\epsilon / K) \leq\left|\frac{1}{\phi^{\prime}(0)}\right| \leq 1+O(\epsilon / K) .
$$

Assim existe $C>0$ tal que

$$
||(\phi)^{\prime}(0)|-1| \leq C\left|\frac{1}{\left|(\phi)^{\prime}(0)\right|}-1\right| \leq O(\epsilon / K) .
$$

De Eq. (1.3) para $|w| \leq \epsilon$ temos

$$
\left|(\phi)^{\prime}(w)\right| \leq 1+O(\epsilon / K)
$$

Aplicando o Corolário 1.13 para $\varphi$ obtemos

$$
\left|\arg \left(\phi^{\prime}(K z)\right)-\arg \left(\phi^{\prime}(0)\right)\right| \leq 2 \log \left(\frac{1+|z|}{1-|z|}\right) \text { para }|z|<1 .
$$

Como $\phi^{\prime}(0) \in \mathbb{R}$ temos $\arg \left(\phi^{\prime}(0)\right)=0$. Para $|w| \leq \epsilon$ tome $z=w / K$ então

$$
\left|\arg \left(\phi^{\prime}(w)\right)\right| \leq 2 \log \left(\frac{1+\frac{\epsilon}{K}}{1-\frac{\epsilon}{K}}\right)=O(\epsilon / K) .
$$


De Eq. (1.5) e Eq. (1.7) obtemos

$$
\begin{aligned}
\left|\phi^{\prime}(w)-1\right| & =|| \phi^{\prime}(w)\left|e^{i \arg \left(\phi^{\prime}(w)\right)}-1\right| \\
& =\left|\left(\left|\left(\phi^{\prime}(w)\right)\right|-1\right) e^{i \arg \left(\phi^{\prime}(w)\right)}+e^{i \arg \left(\phi^{\prime}(w)\right)}-1\right| \\
& \leq||\left(\phi^{\prime}(w)\right)|-1|+\left|e^{i \arg \left(\phi^{\prime}(w)\right)}-1\right| \\
& \leq O(\epsilon / K) .
\end{aligned}
$$

Por tanto

$$
\begin{aligned}
|\phi-\mathrm{id}|_{B(0, \epsilon)} & =\left|\int_{1}^{z}\left(\phi^{\prime}(w)-1\right) d w\right| \\
& \leq \int_{1}^{z}\left|\left(\phi^{\prime}(w)-1\right)\right||d w| \\
& \leq O(\epsilon / K) .
\end{aligned}
$$

Agora apresentamos o Teorema Generalizado de Distorção, o qual é uma extensão do Teorema de Distorção.

Teorema 1.15 ([13]) Se E é um subconjunto compacto de uma regiãa $D \subset \mathbb{C}$, então existe uma constante $C$ (dependendo de E) tal que para qualquer aplicação univalente $\varphi: D \rightarrow \mathbb{C}$ e qualquer par de pontos $z, w \in E$ temos

$$
\frac{1}{C} \leq \frac{\left|\varphi^{\prime}(z)\right|}{\left|\varphi^{\prime}(w)\right|} \leq C
$$

Definimos o conjunto de números complexos cuja distância ao intervalo $[-1,1]$ é no máximo $\rho>0$ denotando-o por

$$
D_{\rho}=\{z \in \mathbb{C}: \operatorname{dist}(z,[-1,1])<\rho\}
$$

Será chamado de conjunto $\rho$-estádio .

O seguinte lemma diz quando é possível compor duas aplicações univalentes sobre um domínio com modulo positivo não pequeno. Este resultado será importante para provar na seguinte seção que uma certa família de aplicações analíticas é pre-compacta. 
Lema 1.16 ([1]) Seja $C>0$ e $E_{0} \supset E_{1} \supset[-1,1]$ dominios estritamente encaixados no plano complexo. Existe uma constante $K>0$ tal que o seguinte se satisfaz. Sejam $\phi: E_{0} \rightarrow \mathbb{C}$ e $\psi: E_{\psi} \rightarrow \mathbb{C}$ aplicações univalentes onde $E_{\psi} \subset E_{1}$ e além disso cada uma das aplicações fixam o intervalo $[-1,1]$ e $|\phi-\mathrm{id}|_{E_{0}} \mid \leq C$. Existe um $\rho(\phi)$-estádio $D_{\rho(\phi)} \subset E_{\psi}$ tal que

- $\phi\left(D_{\rho(\phi)}\right) \subset E_{\psi}$,

- $\rho(\phi) \geq e^{-K|\phi-\mathrm{id}|_{E_{0}}} \rho(\psi)$, onde $\rho(\psi)$ é a distancia entre a fronteira de $E_{\psi}$ ao intervalo $[-1,1]$.

Em particular, $\psi \circ \phi$ está definido em $D_{\rho(\phi)}$.

Demonstração. Seja $D_{\rho} \subset E_{0}$ um $\rho$-estádio. A prova será dividida em dois casos. Suponha primeiramente que existe $\widetilde{C}>1000$ tal que $\rho(\psi)>\widetilde{C}$. Assim, se $z \in \partial D_{\rho}$ temos o seguinte

$$
\operatorname{dist}(\phi(z),[-1,1]) \leq \operatorname{dist}(\phi(z), z)+\operatorname{dist}(z,[-1,1]) \leq|\phi-\mathrm{id}|_{E_{0}}+\rho
$$

então

$$
\phi\left(D_{\rho}\right) \subseteq D_{|\phi-\mathrm{id}|_{E_{0}}+\rho}
$$

Tomemos $\rho(\phi)>0$ tal que

$$
\rho(\psi)=\rho(\phi)+|\phi-\mathrm{id}|_{E_{0}} .
$$

Como $\rho(\psi)>\widetilde{C}$ assim

$$
\rho(\phi) \geq\left(1-\frac{1}{\widetilde{C}}|\phi-\mathrm{id}|_{E_{0}}\right) \rho(\psi)
$$

Então obtemos $K>0$ tal que

$$
\rho(\phi) \geq e^{-K|\phi-\mathrm{id}|_{E_{0}}} \rho(\psi)
$$

Finalmente supomos que $\rho(\psi) \leq 1000$. Seja $x \in[-1,1]$. Fazendo uso do Teorema 1.15 existe $C>0$ tal que para todo $z, x \in D_{\rho(\psi)}$

$$
e^{-C|\phi-\mathrm{id}|_{E_{0}}} \leq \frac{|D \phi(z)|}{|D \phi(x)|} \leq e^{C|\phi-\mathrm{id}|_{E_{0}}} .
$$


Por outro lado existe $x_{0} \in[-1,1]$ tal que $D \phi\left(x_{0}\right)=1$ e pelo Teorema do Valor Médio sobre $E_{0}$ temos que para todo $z \in D_{\rho}$ e $x \in[-1,1]$

$$
|\phi(z)-\phi(x)| \leq e^{C|\phi-\mathrm{id}|_{E_{0}}}|z-x|
$$

Para todo $z \in \partial D_{\rho}$ existe $x \in[-1,1]$ tal que $|z-x|=\rho$. Então

$$
\operatorname{dist}(\phi(z),[-1,1]) \leq e^{C|\phi-\mathrm{id}|_{E_{0}}} \rho
$$

para todo $z \in D_{\rho}$. Tome $\rho(\phi)=e^{-C|(\phi-\mathrm{id})|_{E_{0}}} \rho(\psi)$. Isto finaliza a prova.

\subsection{Cotas reais e complexas a priori}

Apresentamos a ferramenta principal para o desenvolvimento deste trabalho, a chamada cota complexa a priori (complex bounds): existe um domínio complexo $V \supset[-1,1]$ tal que para $n$ suficientemente grande a primeira componente de $\widetilde{\mathcal{R}}^{n}(\phi, t)$, onde $(\phi, t) \in \mathcal{U}$ satisfaz condições apropriadas, é bem definida e univalente em $V$.

A cota complexa a priori tem tido muitas aplicações no estudo do operador de renormalização $\mathcal{R}_{2 n}, n \in \mathbb{N}$. Uma das mais importantes aplicações é a convergência do operador de renormalização no conjunto de aplicações infinitamente renormalizáveis e a hiperbolicidade deste operador num espaço apropriado. Sullivan [6] introduziu e mostrou esta propriedade para aplicações infinitamente renormalizáveis com combinatória limitada.

Outros resultados relacionados, para aplicações unimodais infinitamente renormalizáveis com combinatória não limitada, foram dados por Lyubich [14], Lyubich e Yampolsky [15], Graczyk e Swiatek [11], Levin e van Strien [10]. Smania [4] provou "complex bounds" para aplicações analíticas multimodais, infinitamente renormalizáveis, com combinatória limitada. Recentes resultados foram obtidos por Khan, Lyubich and Kozlowski, Strien and Shen.

Aqui obtemos uma cota complexa a priori para a primeira componente univalente do operador de renormalização $\widetilde{\mathcal{R}}_{\alpha}$ olhando na decomposição de difeomorfismos da primeira componente do operador. Esta ferramenta é útil porque nos permite definir, no Capítulo 3 , 
o operador de renormalização complexo $\widetilde{R}_{\alpha}$, onde o expoente crítico é $\alpha>1$. Lembremos que o espaço de Banach $\mathrm{C}^{3}([-1,1], \mathbb{R})$ é o conjunto das aplicações $\mathrm{C}^{3}$ dotadas com a norma do supremo

$$
|f|_{\mathrm{C}^{3}([-1,1])}=\sup _{x \in[-1,1]}\left\{|f(x)|,|D f(x)|,\left|D^{2} f(x)\right|\right\}
$$

Teorema 1.17 (Complex Bounds) Seja $B>0$ uma constante. Existe $\delta_{0}=\delta_{0}(B)>$ 0, tal que o seguinte se satisfaz: para cada conjunto aberto convexo $V \subset \mathbb{C}$ simétrico com respeito ao eixo real contendo o intervalo $I=[-1,1]$ e para todo $C>0$, existe $N=N(B, C, V) \geq 1$ tal que se

- $\phi: V \rightarrow \mathbb{C}$ é uma aplicação univalente definida em $V \subset \mathbb{C}$.

- $|\phi|_{\mathrm{C}^{3}([-1,1])} \leq C, e$

- $(\phi, t) \in \mathcal{U}$ é $M$-vezes renormalizável com combinatória limitada por $B$, para $M>N$.

Então para todo $M>n \geq N$ a primeira componente de $\widetilde{\mathcal{R}}^{n}(\phi, t)$, está definida e é univalente no domínio complexo $D_{3 \delta_{0}}$.

Antes de entrar nos detalhes da prova deste teorema derivamos os seguintes corolários. Nós apresentamos a prova do Teorema 1.17 no que resta desta seção.

Corolário 1.18 Para todo $B>0$ existe $\delta_{0}(B)>0$, e $N=N\left(B, C, \delta_{0}\right) \geq 1$ tal que se

- $\phi: D_{\delta_{0} / 2} \rightarrow \mathbb{C}$ é uma aplicação univalente definida em $D_{\delta_{0} / 2} \subset \mathbb{C}$.

- $|\phi|_{\mathrm{C}^{3}([-1,1])} \leq C$,

- $(\phi, t) \in \mathcal{U}$ é $M$-vezes renormalizável com combinatória limitada por $B$, para $M>N$.

Então se $M>n \geq N$ temos que a primeira componente de $\widetilde{\mathcal{R}}^{n}(\phi, t)$, está definida e é univalente no domínio complexo $D_{2 \delta_{0}}$. 
Demonstração. Tomemos $V=D_{\delta_{0} / 2}$ e $C$ como o supremo dos $|\phi|_{C^{3}([-1,1])}$, onde $\phi$ é aplicação univalente em $D_{\delta_{0} / 2}$, e é tal que deixa invariante o intervalo $[-1,1] \operatorname{com} \phi(-1)=$ -1 e $\phi(1)=1$. A escolha do $C$ é possível em vista que as aplicações univalentes $\phi$ definidas sobre $D_{\delta_{0} / 2} \backslash\{-1,1\}$ formam uma família normal pelo Teorema de Montel [12]. Assim como conseqüência direta do Teorema 1.17 tem-se o resultado.

Definição 1.19 Dizemos que um par $(\phi, t)$ é unimodal se

- Existe uma vizinhança $V$ do intervalo $I=[-1,1]$ tal que $\varphi: V \rightarrow \mathbb{C}$ é uma aplicação holomorfa com extensão contínua em $\bar{V}$, onde $\varphi(-1)=-1, \varphi(1)=1$, e $t \in \mathbb{C}$,

- $\phi$ é univalente em $V$,

- $\phi$ é real na reta real e $t \in[0,1]$.

Corolário 1.20 Para cada par $(\phi, t)$ unimodal, infinitamente renormalizável com combinatória limitada por $B>0$, existe $N$ tal que a sequência formada pelas primeiras componentes dos pares $\widetilde{\mathcal{R}}^{n}(\phi, t)$, com $n \geq N$, forma uma família pré-compacta em $D_{2 \delta_{0}}$.

Demonstração. A parte difeomorfa de cada $\widetilde{\mathcal{R}}^{n}(\phi, t)$ é um difeomorfismo o qual aplica o intervalo $[-1,1]$ sobre se mesmo. Na verdade este difeomorfismo analítico é a decomposição de difeomorfismos.

Pelo Teorema 1.17 a parte difeomorfa de cada $\widetilde{\mathcal{R}}^{n}(\phi, t)$, onde $n \in \mathbb{N}$, tem extensão univalente sobre um $\delta_{0}$-estádio fixo $D_{\delta_{0}}$ contendo o intervalo $[-1,1]$ o qual não depende de $n$. Desde que cada uma destas transformações univalentes mantém fixo - 1 e 1 , segue do Teorema de Montel que com a norma do supremo sobre todas as funções holomorfas temos que as primeiras componentes dos pares $\widetilde{\mathcal{R}}_{n}(\phi, t)$ forma uma família pré-compacta em $D_{2 \delta_{0}}$.

Um ingrediente especial na prova do Teorema Complex Bound é dado no seguinte lema onde estabelecemos a cota real a priori (real bounds) o qual precisamos para obter controle sobre a geometria dos ciclos de pares $N$-vezes renormalizáveis, para $N$ suficientemente grande, com combinatória limitada por uma constante $B>0$. 
Nós podemos seguir a prova deste resultado de W. de Melo e van Strien [19], ver Teorema 2.1, Capitulo VI. Primeiro fixemos o expoente crítico $\alpha>1$.

Lema 1.21 (Real bounds) Seja $B>0$ uma constante. Então existe $0<b<1$ com $a$ seguinte propriedade: para todo $C>0$, existe $N=N(B, C) \geq 1$ tal que se $(\phi, t) \in \mathcal{U}$ é $M$-vezes renormalizável com combinatória limitada por $B$ com $M>N$, e $|\phi|_{\mathrm{C}^{3}([-1,1])} \leq C$, temos que

1. Se $I_{i_{l}}^{n+1} \subset I_{j}^{n}, l=1, \cdots, m_{n}$ são os intervalos do $(n+1)$-ésimo ciclo de renormalização, onde $N \leq n<M$, que estão contidos no intervalo $I_{j}^{n}$ do n-ésimo ciclo então

$$
b<\frac{\left|I_{i_{l}}^{n+1}\right|}{\left|I_{j}^{n}\right|}<1-b
$$

onde $l=1, \cdots, m_{n}$, para todo $N \leq n<M$.

2. Se J é uma componente conexa de $I_{j}^{n} \backslash \bigcup_{l=1}^{m_{n}} I_{i_{l}}^{n+1}$ então

$$
b<\frac{|J|}{\left|I_{j}^{n}\right|}<1-b,
$$

para todo $N \leq n<M$.

Observação 1.22 Seja $\alpha>1$ e $\delta>0$ suficientemente pequeno. Nós podemos supor que a constante $b<1$ do Lema 1.21 é a mesma para os pares $(\phi, t) \in \mathcal{U}$, M-vezes renormalizáveis com combinatória limitada por $B$, para $M>N$ suficientemente grande, com ordem crítico $\widetilde{\alpha}$, onde $|\alpha-\widetilde{\alpha}|<\delta$.

Assim podemos estabelecer o seguinte resultado.

Teorema 1.23 Seja $B>0, \alpha>1, e C>0$. Existe $\delta>0 e \varepsilon=\varepsilon(\delta)>0$, tal que o seguinte se satisfaz: para cada domínio complexo $V$ contendo o intervalo $[-1,1]$ existe $N=N(B, \alpha, C, V) \geq 1$ tal que 
- se $(\phi, t) \in \mathcal{U}$ é $M$-vezes renormalizável com combinatória limitada por $B$, para $M>N$ suficientemente grande, com ordem crítico $\widetilde{\alpha}$, com $|\alpha-\widetilde{\alpha}|<\delta$ e $\phi$ aplicação univalente definida sobre $V, e$

- $|\phi|_{\mathrm{C}^{3}([-1,1])} \leq C$,

Então para todo $N \leq n<M$ as aplicações $\phi_{0}^{n}=Z_{\phi^{-1}\left(I_{1}^{n}\right)}(\phi), q_{j}^{n}=Z_{I_{j}^{n}}\left(q_{t}\right)$ e $\phi_{j}^{n}=$ $Z_{\phi^{-1}\left(I_{j+1}^{n}\right)}(\phi)$, onde $j=1, \cdots, q_{n}-1$, têm extensões univalentes para aplicações definidas sobre um $\varepsilon$-estádio $D_{\varepsilon}$.

Demonstração. Seja $\delta>0$ e $N \geq 1$ como na Observação 1.22. Considere os setores no plano complexo denotado por

$$
S_{a}^{+}=\left\{z \in \mathbb{C}:|\arg (z)|<\frac{\pi}{a}\right\}
$$

e

$$
S_{a}^{-}=\left\{z \in \mathbb{C}:|\arg (-z)|<\frac{\pi}{a}\right\},
$$

onde $a>0$. Suponha que o par $(\phi, t) \in \mathcal{U}$ satisfaz as hipóteses do Teorema. Fixemos $\mathcal{I}^{n}=\left\{I_{1}^{n}, I_{2}^{n}, \ldots, I_{q}^{n}\right\}$ o ciclo correspondente à $n$-ésima renormalização, $N \leq n \leq M$. Denotemos por $x_{j}^{n}$ o ponto da fronteira do intervalo $I_{j}^{n}, j \neq 0$, mais próximo ao ponto crítico. Existe um nível de renormalização entre $N$ e $n$ tal que o intervalo que contém o ponto crítico neste nível contém o intervalo $I_{j}^{n}$. Assim escolha o primeiro $k>0$ tal que $I_{0}^{n-k} \supset I_{j}^{n}$, onde $N \leq n-k<n$. Então $I_{j}^{n}$ não está contido em $I_{0}^{n-k+1}$. Nós temos dois casos:

Caso I. Primeiro quando $k=1$ (ver a figura 1.2).

Pelo Lema 1.21 obtemos

$$
\begin{aligned}
\frac{\operatorname{dist}\left(0, x_{j}^{n}\right)}{\left|I_{j}^{n}\right|} & \geq \frac{\left|I_{0}^{n}\right|}{2\left|I_{j}^{n}\right|} \\
& =\frac{\left|I_{0}^{n}\right|}{2\left|I_{0}^{n-1}\right|} \cdot \frac{\left|I_{0}^{n-1}\right|}{\left|I_{j}^{n}\right|} \\
& >\frac{b}{2} \cdot \frac{1}{(1-b)} .
\end{aligned}
$$




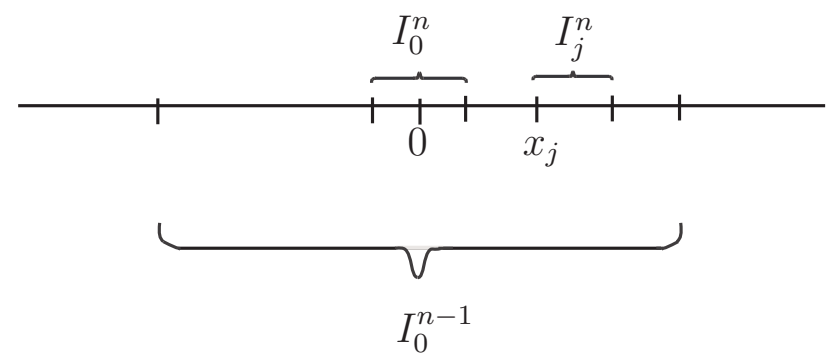

Figura 1.2: Caso $k=1, I_{0}^{n-1} \supset I_{j}^{n}$.

Caso II. Para $k>1$ (ver a figura 1.3). Podemos ver que $I_{j}^{n}$ está contido em algum intervalo $I_{j(n-k+1)}^{n-k+1} \subset I_{0}^{n-k}-I_{0}^{n-k+1}$. Na verdade o intervalo $I_{j}^{n}$ está contido em uma sequência encaixada de intervalos de níveis inferiores. Assim $I_{j}^{n} \subset I_{j(n-1)}^{n-1} \subset \cdots I_{j(n-k+2)}^{n-k+2} \subset I_{j(n-k+1)}^{n-k+1}$ e pelo Lema 1.21 temos

$$
\left|I_{j}^{n}\right|<(1-b)^{k-1}\left|I_{j(n-k+1)}^{n-k+1}\right| \leq \frac{(1-b)^{k-1}}{2} \cdot\left(\left|I_{0}^{n-k}\right|-\left|I_{0}^{n-k+1}\right|\right) .
$$

De Eq.( 1.10) obtemos

$$
\begin{aligned}
\frac{\operatorname{dist}\left(0, x_{j}^{n}\right)}{\left|I_{j}^{n}\right|} & \geq \frac{\left|I_{0}^{n-(k-1)}\right|}{2\left|I_{j}^{n}\right|} \\
& >\frac{1}{(1-b)^{k-1}} \cdot \frac{\left|I_{0}^{n-(k-1)}\right|}{\left|I_{0}^{n-k}\right|-\left|I_{0}^{n-k+1}\right|} \\
& >\frac{1}{(1-b)^{k-1}} \cdot \frac{\left|I_{0}^{n-(k-1)}\right|}{\left|I_{0}^{n-k}\right|} \\
& >\frac{b}{(1-b)^{k-1}} \\
& \geq \frac{b}{1-b}
\end{aligned}
$$

Em ambos os casos obtemos

$$
\frac{\operatorname{dist}\left(0, x_{j}^{n}\right)}{\left|I_{j}^{n}\right|}>\frac{b}{2(1-b)}
$$




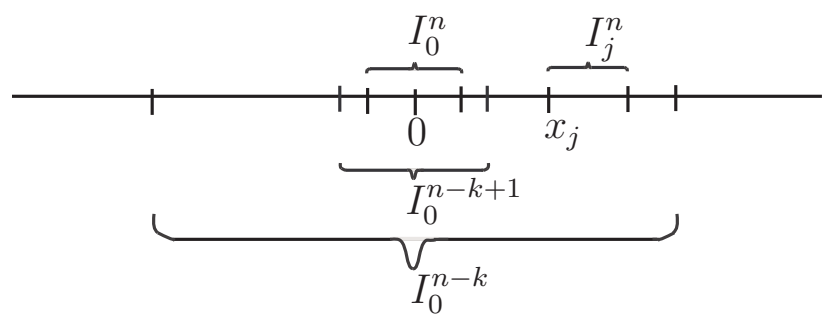

Figura 1.3: Caso $k>1, I_{0}^{n-k} \supset I_{j}^{n} \operatorname{mas} I_{j}^{n}$ não está contido em $I_{0}^{n-k+1}$.

Com esta estimativa, podemos definir os difeomorfismos $\phi_{0}^{n}, q_{j}^{n}$ e $\phi_{j}^{n}$, para $j=1, \cdots, q_{n}-1$, em um domínio comum no plano complexo. De fato, nós sabemos que o ramo principal da função logaritmo log é holomorfo sobre o conjunto $\mathbb{C} \backslash\{z \in \mathbb{R}: z \leq 0\}$. Sejam $q_{t}^{+}: S_{\widetilde{\alpha}}^{+} \rightarrow \mathbb{C}$ e $q_{t}^{-}: S_{\widetilde{\alpha}}^{-} \rightarrow \mathbb{C}$ aplicações univalentes onde

$$
q_{t}^{+}(z)=-2 t e^{\widetilde{\alpha} \log z}+2 t-1
$$

$\mathrm{e}$

$$
q_{t}^{-}(z)=-2 t e^{\widetilde{\alpha} \log (-z)}+2 t-1 .
$$

Seguimos a prova definindo um domínio comum para as aplicações $q_{j}^{n}$, para $j=1, \cdots, q_{n}-$ 1, tomando en conta dos domínios diferentes para o expoente crítico $\alpha>1$.

Primeiramente quando $\alpha \in(1,2)$. Para $I_{j}^{n} \subset S_{\widetilde{\alpha}}^{+}\left(\right.$ou $\left.I_{j}^{n} \subset S_{\widetilde{\alpha}}^{-}\right)$aplicando o operador zoom $Z_{I_{j}^{n}}$ para o difeomorfismo $\left.q_{t}^{+}\right|_{I_{j}^{n}}\left(\right.$ ou $\left.\left.q_{t}^{-}\right|_{I_{j}^{n}}\right)$. Assim podemos definir $q_{j}^{n}=Z_{I_{j}^{n}}\left(q_{t}^{+}\right)$ (ou $\left.q_{j}^{n}=Z_{I_{j}^{n}}\left(q_{t}^{-}\right)\right)$sobre o conjunto $\epsilon_{1}$-estádio

$$
D_{\epsilon_{1}}=\left\{z \in \mathbb{C}: \operatorname{dist}(z,[-1,1])<\epsilon_{1}\right\},
$$

onde

$$
\epsilon_{1}=\frac{b}{2(1-b)}
$$

Este conjunto contém o intervalo $[-1,1]$.

Agora para $\alpha \geq 2$. Consideremos a distancia $a_{j}$ do ponto fronteira $x_{j}^{n}$ do intervalo $I_{j}^{n} \subset S_{\widetilde{\alpha}}^{+}$ (ou $I_{j}^{n} \subset S_{\widetilde{\alpha}}^{-}$) á fronteira do setor $S_{\widetilde{\alpha}}^{+}\left(\right.$ou $\left.S_{\widetilde{\alpha}}^{-}\right)$. Um cálculo geométrico elementar leva a 

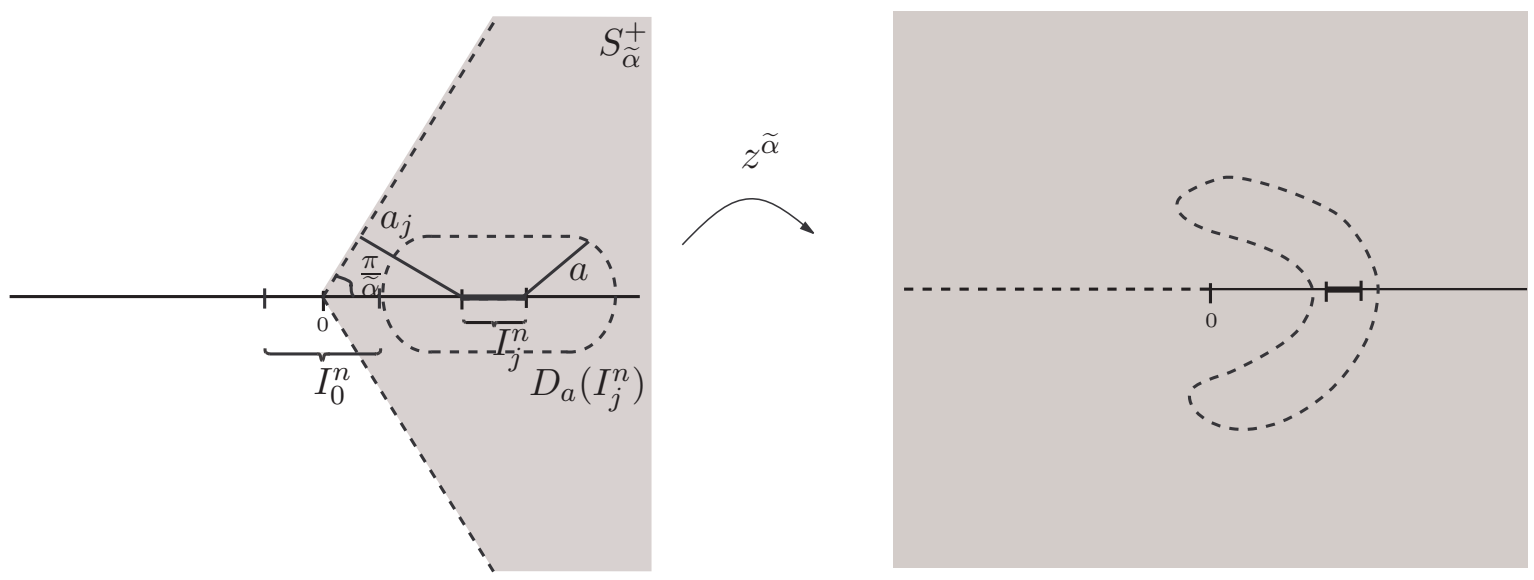

Figura 1.4: $a_{j}>a=\sin \left(\frac{\pi}{\alpha-\delta}\right) \cdot \frac{b}{2(1-b)}\left|I_{j}^{n}\right|$.

seguinte relação

$$
a_{j}=\sin \left(\frac{\pi}{\widetilde{\alpha}}\right) \cdot \operatorname{dist}\left(0, x_{j}^{n}\right)>\sin \left(\frac{\pi}{\alpha-\delta}\right) \cdot \frac{b}{2(1-b)}\left|I_{j}^{n}\right|,
$$

veja figura 1.4. Faça um zoom do difeomorfismo $\left.q_{t}^{+}\right|_{I_{j}^{n}}\left(\right.$ ou $\left.\left.q_{t}^{-}\right|_{I_{j}^{n}}\right)$. Em este caso tome

$$
\epsilon_{1}=\sin \left(\frac{\pi}{\alpha-\delta}\right) \cdot \frac{b}{2(1-b)}
$$

assim podemos definir $q_{j}^{n}=Z_{I_{j}^{n}}\left(q_{t}^{+}\right)$(ou $\left.q_{j}^{n}=Z_{I_{j}^{n}}\left(q_{t}^{-}\right)\right)$sobre um conjunto $\epsilon_{1}$-estádio $D_{\epsilon_{1}}$. Denotemos por $\epsilon_{j}^{n}$ a distancia do intervalo $\phi^{-1}\left(I_{j+1}^{n}\right)$ à fronteira de $V$. É claro que

$$
\epsilon_{j}^{n} \geq \operatorname{dist}([-1,1], \partial V)
$$

para todo $j=0, \cdots, q_{n}-1$. Existe $N_{0} \geq N$ e $K>1000$ suficientemente grande tal que para todo $N_{0} \leq n<M$ temos

$$
\operatorname{dist}([-1,1], \partial V) \geq K\left|\phi^{-1}\left(I_{j+1}^{n}\right)\right|,
$$

onde $j=0, \cdots, q_{n}-1$. Então $\phi_{j}^{n}=Z_{\phi^{-1}\left(I_{j+1}^{n}\right)}(\phi)$, para todo $j=0, \cdots, q_{n}-1$ e para todo $N_{0} \leq n<M$, estão definidos sobre

$$
D_{K}=\{z \in \mathbb{C}: \operatorname{dist}(z,[-1,1])<K\}
$$


Escolha $\varepsilon<\epsilon_{1}$. Nós vemos que $\phi_{0}^{n}$, e as aplicações $q_{j}^{n}, \phi_{j}^{n}$, para todo $j=1, \cdots, q_{n}-1$, estão definidas sobre o $\varepsilon$-estádio

$$
D_{\varepsilon}=\{z \in \mathbb{C}: \operatorname{dist}(z,[-1,1])<\varepsilon\}
$$

para todo $N_{0} \leq n<M$.

Observação $\mathbf{1 . 2 4}$ Nós podemos observar o seguinte:

1. Note que quando o expoente crítico $\alpha \in(1,2)$. Nós podemos definir as aplicações $q_{j}^{n}=Z_{I_{j}^{n}}\left(q_{t}^{+}\right)\left(\right.$or $\left.q_{j}^{n}=Z_{I_{j}^{n}}\left(q_{t}^{-}\right)\right)$, para todo $j=1, \cdots, q_{n}-1$, sobre um domínio comum que é a bola

$$
B\left(0, \frac{1}{2}+\epsilon_{1}\right)=\left\{z \in \mathbb{C}: \operatorname{dist}(z, 0)<\frac{1}{2}+\epsilon_{1}\right\}
$$

onde

$$
\epsilon_{1}=\frac{b}{2(1-b)}
$$

que contém o intervalo $[-1,1]$.

Proposição 1.25 Seja $(\phi, t) \in \mathcal{U}$ como no enunciado do Teorema 1.23. Existem $N_{0}>0$, $L>0, H>0$ e $b<1$ tal que as aplicações $\phi_{0}^{n}=Z_{\phi^{-1}\left(I_{1}^{n}\right)}(\phi), q_{j}^{n}=Z_{I_{j}^{n}}\left(q_{t}\right)$ e $\phi_{j}^{n}=$ $Z_{\phi^{-1}\left(I_{j+1}^{n}\right)}(\phi)$, onde $j=1, \cdots, q_{n}-1$, com extensões univalentes a aplicações definidas sobre um dominio $D_{\varepsilon}$ contendo o intervalo $[-1,1]$, satisfazem

$$
\sum_{j=0}^{q_{n}-1}\left|\phi_{j}^{n}-\mathrm{id}\right|_{D_{\varepsilon}} \leq L b^{n},
$$

$e$

$$
\sum_{I_{j}^{n} \in L_{k}^{n}}\left|q_{i}^{n}-\mathrm{id}\right|_{D_{\varepsilon}} \leq H(1-b)^{k-1}
$$

para todo $N_{0} \leq n<M$.

Demonstração. Seja $R=\operatorname{dist}([-1,1], \partial V)$. Para a primeira estimativa definimos

$$
K=K(j, n)=\frac{R}{\left|\phi^{-1}\left(I_{j+1}^{n}\right)\right|} .
$$


Existe $N_{0}>1$ tal que para todo $N_{0} \leq n<M$ temos $0<1+\varepsilon<K / 4$ e

$$
\phi_{j}^{n}=Z_{\phi^{-1}\left(I_{j+1}^{n}\right)}(\phi)
$$

onde $j=0, \cdots, q_{n}-1$, são definidas sobre a bola $B(0, K / 2)$. De acordo com o Teorema 1.14

$$
\left|\phi_{j}^{n}-\mathrm{id}\right|_{D_{\varepsilon}} \leq O\left(\frac{1+\varepsilon}{K}\right) .
$$

Por outro lado pela cota real a priori existem constantes $L_{1}>0$ e $b<1$ tal que

$$
\sum_{j=1}^{q_{n}-1}\left|I_{j}^{n}\right| \leq L_{1} b^{n},
$$

para todo $N_{0} \leq n<M$. Como o difeomorfismo $\phi$ tem derivada limitada estas duas constantes podem ser ajustadas de modo que

$$
\sum_{j=1}^{q_{n}-1}\left|\phi^{-1}\left(I_{j}^{n}\right)\right| \leq L_{1} b^{n},
$$

para todo $N_{0} \leq n<M$. Assim temos

$$
\begin{aligned}
\sum_{j=0}^{q_{n}-1} \frac{1+\varepsilon}{K} & =(1+\varepsilon) \sum_{j=0}^{q_{n}-1} \frac{\left|\phi^{-1}\left(I_{j+1}^{n}\right)\right|}{R} \\
& \leq \frac{(1+\varepsilon)}{R} L b^{n}
\end{aligned}
$$

o qual implica a Eq. (1.11).

Para a segunda estimativa tratamos dois casos:

Caso I. Para $k>1$ no Caso II do Teorema 1.23 podemos definir para $I_{j}^{n} \subset I_{0}^{n-k} \backslash I_{0}^{n-k+1}$

$$
K_{1}=K_{1}(j, n)= \begin{cases}\frac{\left|I_{0}^{n-k+1}\right|}{2\left|I_{j}^{n}\right|} & \text { se } 1<\alpha<2 \\ \sin \left(\frac{\pi}{\alpha-\delta}\right) \cdot \frac{\left|I_{0}^{n-k+1}\right|}{2\left|I_{j}^{n}\right|} & \text { se } \quad \alpha \geq 2\end{cases}
$$

Observe que as aplicações univalentes

$$
q_{j}^{n}=Z_{I_{j}^{n}}\left(q_{t}\right),
$$


onde $j=1, \cdots, q_{n}-1$, são definidas na bola $B\left(0, K_{1} / 2\right)$. Nós temos $1+\varepsilon<K_{1} / 4$ então pelo Teorema 1.14

$$
\left|q_{j}^{n}-\mathrm{id}\right|_{D_{\varepsilon}} \leq O\left(\frac{1+\varepsilon}{K_{1}}\right)
$$

Por outro lado obtemos

$$
\begin{aligned}
\sum_{I_{j}^{n} \subset I_{0}^{n-k} \backslash I_{0}^{n-k+1}} \frac{1+\varepsilon}{K_{1}} & \leq(1+\varepsilon) \sum_{I_{j}^{n} \subset I_{0}^{n-k} \backslash I_{0}^{n-k+1}} \frac{2\left|I_{j}^{n}\right|}{\left|I_{0}^{n-k+1}\right|} \\
& \leq 2(1+\varepsilon) \cdot \frac{(1-b)^{k-1}\left(\left|I_{0}^{n-k}\right|-\left|I_{0}^{n-k+1}\right|\right)}{\left|I_{0}^{n-k+1}\right|} \\
& \leq \frac{2(1+\varepsilon)}{b} \cdot(1-b)^{k-1} \cdot
\end{aligned}
$$

Caso II. Para $k=1$. Do Teorema 1.23, para o intervalo $I_{j}^{n} \subset I_{0}^{n-1}$, onde $N \leq n-k<$ $n<M$, as aplicações univalentes $q_{j}^{n}=Z_{I_{j}^{n}}\left(q_{t}\right)$ são definidas no $\epsilon_{1}$-estádio $D_{\epsilon_{1}}$, onde $\epsilon_{1}>\varepsilon$. Lembremos que $q_{j}^{n}(-1)=-1$ e $q_{j}^{n}(1)=1$. Assim considerando as aplicações univalentes $q_{j}^{n}$ definidas sobre $D_{\epsilon_{1}} \backslash\{-1,1\}$ pelo Teorema de Montel [12] estas formam uma família normal. Então existe $C_{1}>0$ tal que para todo intervalo $I_{j}^{n} \subset I_{0}^{n-1}$, onde $N \leq n-k<n<M$, temos

$$
\left|q_{j}^{n}-\mathrm{id}\right|_{D_{\varepsilon}}<C_{1}
$$

Assim dos dois casos concluímos que existe $H>0$ tal que

$$
\sum_{I_{j}^{n} \in L_{k}^{n}}\left|q_{i}^{n}-\mathrm{id}\right|_{D_{\varepsilon}} \leq H(1-b)^{k-1} .
$$

Agora estamos prontos para apresentar a prova do Teorema de Cotas Complexas. Prova do Teorema 1.17. Pelo Teorema 1.23 as aplicações $\phi_{0}^{n}, q_{i}^{n}$ e $\phi_{i}^{n}$, para todo $i=$ $1, \cdots, q_{n}-1$, tem extensão univalente sobre um conjunto $\varepsilon$-estádio $D_{\varepsilon}$ contendo o intervalo $[-1,1]$. Seja $D_{\varepsilon} \supset D_{\varepsilon / 2} \supset[-1,1]$ conjuntos estritamente encaixados. Defina para cada $j=1, \cdots, q_{n}-1$ os difeomorfismos

$$
\Phi_{j}^{n}=\phi_{q_{n}-1}^{n} \circ q_{q_{n}-1}^{n} \circ \cdots \circ \phi_{q_{n}-j}^{n}
$$


e

$$
\Psi_{j}^{n}=\phi_{q_{n}-1}^{n} \circ q_{q_{n}-1}^{n} \circ \cdots \circ \phi_{q_{n}-j}^{n} \circ q_{q_{n}-j} .
$$

Defina por $\rho\left(\phi_{q_{n}-1}^{n}\right):=\varepsilon / 3$. Vamos a construir por indução os domínios para as aplicações $\Phi_{j}^{n}$ e $\Psi_{j}^{n}$, para $j=1, \cdots, q_{n}-1$. Primeiro seja $j=1$. Pelo Lema 1.16 existe uma constante $K>0$ tal que:

A. Para $q_{q_{n}-j}^{n}: D_{\varepsilon} \rightarrow \mathbb{C}$ e $\Phi_{j}^{n}: D_{\rho\left(\phi_{q_{n}-j}^{n}\right)} \rightarrow \mathbb{C}$ existe um $\rho\left(q_{q_{n}-j}^{n}\right)$-estádio $D_{\rho\left(q_{q_{n}-j}^{n}\right)} \subset$ $D_{\left.\rho_{(} \Phi_{j}^{n}\right)}$ tal que

- $q_{q_{n}-j}^{n}\left(D_{\rho\left(q_{q_{n}-j}^{n}\right)}\right) \subset D_{\rho\left(\Phi_{j}^{n}\right)}$,

- $\rho\left(q_{q_{n}-j}^{n}\right) \geq e^{-k\left|q_{q_{n}-j}^{n}-\mathrm{id}\right|_{D_{\varepsilon}}} \rho\left(\Phi_{j}^{n}\right)$, onde $\rho\left(q_{q_{n}-j}^{n}\right)$ e $\rho\left(\Phi_{j}^{n}\right)$ são as distancias entre a fronteira respectivamente de $D_{\rho\left(q_{q_{n}-j}^{n}\right)}$ e $D_{\rho\left(\Phi_{j}^{n}\right)}=D_{\rho\left(\phi_{q_{n}-j}^{n}\right)}$ ao intervalo $[-1,1]$.

Note que $D_{\rho\left(q_{q_{n}-j}^{n}\right)}$ é o domínio de definição da aplicação $\Psi_{j}^{n}=\Phi_{j}^{n} \circ q_{q_{n}-j}^{n}$. Assim de novo pelo Lema 1.16 existe uma constante $K>0$ tal que:

B. Para $\phi_{q_{n}-j-1}^{n}: D_{\varepsilon} \rightarrow \mathbb{C}$ e $\Psi_{j}^{n}: D_{\rho\left(q_{q_{n}-j}^{n}\right)} \rightarrow \mathbb{C}$ existe um conjunto $\rho\left(\phi_{q_{n}-j-1}^{n}\right)$-estádio $D_{\rho\left(\phi_{q_{n-j-1}^{n}}^{n}\right)} \subset D_{\rho\left(\Psi_{j}^{n}\right)}$ tal que

- $\phi_{q_{n-j-1}}\left(D_{\rho\left(\phi_{q_{n-j-1}^{n}}^{n}\right)}\right) \subset D_{\rho\left(\Psi_{j}^{n}\right)}$

- $\rho\left(\phi_{q_{n}-j-1}^{n}\right) \geq e^{-k\left|\phi_{q_{n-j-1}}^{n}-\mathrm{id}\right|_{D_{\varepsilon}}} \rho\left(\Psi_{j}^{n}\right)$, onde $\rho\left(\phi_{q_{n}-j-1}^{n}\right)$ e $\rho\left(\Psi_{j}^{n}\right)$ são as distâncias entre a fronteira respectivamente de $D_{\rho\left(\phi_{q_{n-j-1}}^{n}\right)}$ e $D_{\rho\left(\Psi_{j}^{n}\right)}=D_{\rho\left(q_{q_{n}-j}^{n}\right)}$ ao intervalo $[-1,1]$.

Aqui $D_{\rho\left(\phi_{q_{n-j-1}}^{n}\right)}$ é o domínio de definição da aplicação $\Phi_{j+1}^{n}=\Psi_{j}^{n} \circ \phi_{q_{n}-j-1}^{n}$.

Então para cada $j=2, \cdots, q_{n}-1$ aplicamos o Lema 1.16 para o par de aplicações em $A$ e $B$. Finalmente obtemos um $\rho\left(\phi_{0}^{n}\right)$-estádio $D_{\rho\left(\phi_{0}^{n}\right)}$ que é domínio de definição da aplicação

$$
\Psi_{q_{n}}^{n} \circ \phi_{0}^{n}=\phi_{q_{n}-1}^{n} \circ q_{q_{n}-1}^{n} \circ \cdots \circ \phi_{1}^{n} \circ q_{1}^{n} \circ \phi_{0}^{n}
$$

onde

$$
\rho\left(\phi_{0}^{n}\right) \geq \prod_{i=1}^{q_{n}-1} e^{-K\left|q_{i}^{n}-\mathrm{id}\right|_{D_{\varepsilon}}} \cdot \prod_{i=1}^{q_{n}-2} e^{-K\left|\phi_{i}^{n}-\mathrm{id}\right|_{D_{\varepsilon}}} \cdot \rho\left(\phi_{q_{n}-1}^{n}\right)
$$


Como $\rho\left(\phi_{q_{n}-1}^{n}\right)=\frac{\varepsilon}{3}$ temos

$$
\begin{aligned}
\rho\left(\phi_{0}^{n}\right) & \geq \frac{\varepsilon}{3} \cdot e^{-K \sum_{i=1}^{q_{n}-1}\left|q_{i}^{n}-\mathrm{id}\right|_{D_{\varepsilon}}} \cdot e^{-K \sum_{i=1}^{q_{n}-2}\left|\phi_{i}^{n}-\mathrm{id}\right|_{D_{\varepsilon}}} \\
& \geq \frac{\varepsilon}{3} \cdot e^{-K \sum_{i}\left|q_{i}^{n}-\mathrm{id}\right|_{D_{\varepsilon}}} \cdot e^{-K \sum_{i}\left|\phi_{i}^{n}-\mathrm{id}\right|_{D_{\varepsilon}}}
\end{aligned}
$$

Pela Proposição 1.25 existem $L>0, H>0$ e $b<1$ tal que

$$
\sum_{I_{j}^{n} \in \mathcal{I}_{k}^{n}}\left|q_{i}^{n}-\mathrm{id}\right|_{D_{\varepsilon}}<H(1-b)^{k-1}
$$

e

$$
\sum_{i}\left|\phi_{i}^{n}-\mathrm{id}\right|_{D_{\varepsilon}}<L b^{n}
$$

para todo $N_{0}<n<M$ e $1 \leq k \leq n$. Segue de (1.17) que

$$
\begin{aligned}
\rho\left(\phi_{0}^{n}\right) & \geq \frac{\varepsilon}{3} \cdot e^{-K \sum_{k=1}^{n} \sum_{I_{j}^{n} \in L_{k}^{n}}\left|q_{i}^{n}-\mathrm{id}\right|_{D_{\varepsilon}}} \cdot e^{-K \sum_{i}\left|\phi_{i}^{n}-\mathrm{id}\right|_{D_{\varepsilon}}} \\
& \geq \frac{\varepsilon}{3} \cdot e^{-K \sum_{k=1}^{\infty} H(1-b)^{k-1}} e^{-K L} \\
& =\frac{\varepsilon}{3} \cdot e^{-K H \frac{1}{b}-K L}
\end{aligned}
$$

para todo $N_{0}<n<M$. Tomando $3 \delta_{0}=\frac{\varepsilon}{3} \cdot e^{-K H \frac{1}{b}-K L}$ temos que a primeira componente da família $\widetilde{\mathcal{R}}^{n}(\phi, t)$ tem uma extensão univalente sobre o domínio $D_{3 \delta_{0}}$ que não depende de $n$. 


\section{Capítulo 2}

\section{Operador Composição}

Na primeira seção definimos alguns espaços de Banach complexos e seus respectivos espaços tangentes. Estes são importantes para definir o operador composição $L$, o qual relaciona o operador de renormalização $\mathcal{R}$ com o novo operador de renormalização $\widetilde{\mathcal{R}}$ definidos no Capítulo 1, quando $\alpha=2 n$. As seguintes seções mostram propriedades do operador $L$, a ser definido na Seção 2.2, tais como:

- injetividade (veja Proposição 2.5),

- injetividade de $\mathcal{D} L$ (veja Proposição 2.6),

- $\mathcal{D} L$ tem imagem densa (veja Lema 2.7), e

- compacidade de $\mathcal{D} L$ (veja Lema 2.8).

Isto permite concluir no seguinte capítulo que o espectro do ponto fixo $\left(\phi^{*}, t^{*}\right)$ de $\widetilde{\mathcal{R}}_{2 n}$ coincide com o espectro do ponto fixo $\phi^{*} \circ q_{t^{*}}$ de $\mathcal{R}_{2 n}$. 


\subsection{Espaços de Banach e seus espaços tangentes.}

Seja $\mathcal{B}_{V}$ o espaço de Banachcomplexo de aplicações holomorfas $f$ definidas numa vizinhança $V$ com uma extensão continua em $\bar{V}$, dotada com a norma do supremo. Seja $\mathcal{A}_{V}$ o conjunto de aplicações holomorfas $\varphi: V \rightarrow \mathbb{C}$ com extensão contínua em $\bar{V}$, onde $\varphi(-1)=-1$ e $\varphi(1)=1$. Este é um subespaço afim de $\mathcal{B}_{V}$.

Denote por $\mathcal{T}_{V}$ o espaço de Banach complexo de aplicações holomorfas $\omega \in \mathcal{B}_{V}$ da forma $\omega=\psi\left(x^{2 n}\right)$ em uma vizinhança de $[-1,1]$, com $\omega(-1)=\omega(1)=0$, dotada com a norma do supremo. Seja $\mathcal{U}_{V}$ o conjunto de aplicações holomorfas $f: V \rightarrow \mathbb{C}$ contínuas em $\bar{V}$ da forma $f=\psi\left(x^{2 n}\right)$ em uma vizinhança de $[-1,1]$, com $f(-1)=f(1)=-1$. Então $\mathcal{U}_{V}$ é um espaço afim.

Não é difícil verificar que o espaço tangente de $\mathcal{A}_{V}$ em $\varphi$ é $T_{\varphi} \mathcal{A}_{V}=\mathcal{F}_{V}=\left\{\omega \in \mathcal{B}_{V}\right.$ : $\omega(-1)=\omega(1)=0\}$.

Também, é fácil ver que todos os campos de vetores holomorfos $\omega$ no espaço tangente $T_{f} \mathcal{U}_{V}$ são da forma $\omega=\psi\left(x^{2 n}\right)$ numa vizinhança de $[-1,1]$, com $\omega(-1)=\omega(1)=0$. Então $T_{f} \mathcal{U}_{V}=\mathcal{T}_{V}$.

Agora lembremos um resultado de análise complexa. Aqui, int $(K)$ denota o interior do conjunto $K$.

Teorema 2.1 (Aproximação polinomial de Mergelyan [20]) Seja K um subconjunto compacto do plano complexo $\mathbb{C}$ tal que $\mathbb{C} \backslash K$ é conexo. Então, qualquer função contínua $f: K \rightarrow \mathbb{C}$, tal que a restrição $f \mid \operatorname{int}(K)$ é holomorfa, pode ser aproximada uniformemente sobre $K$ por polinômios.

Assim podemos mostrar o seguinte resultado. Para isto dizemos que um conjunto $W \subset \mathbb{C}$ é $2 n$-simétrico, onde $n \in \mathbb{N} \backslash\{0\}$, se $y \in W$, então $x \in W$ para todo $x$ tal que $x^{2 n}=y^{2 n}$.

Lema 2.2 Seja $V \subset \mathbb{C}$ um conjunto aberto convexo tal que é simétrico com respeito ao eixo real contendo o intervalo $[-1,1]$. Seja $f \in \mathcal{U}_{V}$. O conjunto de campos de vetores polinomiais é denso em $T_{f} \mathcal{U}_{V}=\mathcal{T}_{V}$. 
Demonstração. Seja $\omega$ um campo de vetores holomorfo em $\mathcal{T}_{V}$. A idéia da prova é extender o domínio de $\omega$ a um domínio $2 n$-simétrico. Denote por $\lambda=e^{\frac{\pi i}{n}}$ a $2 n$-ésima raiz prima da unidade. Podemos definir para $z \in \lambda^{2 n-i} \bar{V}, i=0, \ldots, 2 n-1$,

$$
\omega(z):=\omega\left(\lambda^{i} z\right)
$$

Afirmacão: $\omega$ é bem definido, uma vez que para $z \in \lambda^{2 n-i} V \cap \lambda^{2 n-j} V, i \neq j$, temos $\omega\left(\lambda^{i} z\right)=\omega\left(\lambda^{j} z\right)$. De fato, existe um $\delta>0$ pequeno tal que para todo $z$ em uma bola de centro 0 e raio $\delta>0$, contido em $\lambda^{2 n-i} V \cap \lambda^{2 n-j} V$, temos

$$
\omega\left(\lambda^{i} z\right)=\omega\left(\lambda^{i} z\right)=\psi\left(\left(\lambda^{i} z\right)^{2 n}\right)=\psi\left(z^{2 n}\right)=\psi\left(\left(\lambda^{j} z\right)^{2 n}\right)=\omega\left(\lambda^{j} z\right) .
$$

Assim pela analiticidade de $\omega$ sobre $\lambda^{2 n-i} V \cap \lambda^{2 n-j} V, i \neq j$, segue a afirmacão. Então

$$
\omega(z)=\sum_{i=0}^{2 n-1} \frac{\omega\left(\lambda^{i} z\right)}{2 n}
$$

para todo $z \in W:=\bigcup_{i=0}^{2 n-1} \lambda^{2 n-i} \bar{V}$. Pelo Teorema 2.1 para qualquer $\epsilon>0$ podemos encontrar um polinômio $p(z)=\sum_{j=0}^{m} a_{j} z^{j}$ tal que

$$
|\omega(z)-p(z)|<\epsilon
$$

para todo $z \in W$. Defina

$$
\widetilde{p}(z):=\sum_{i=0}^{2 n-1} \frac{p\left(\lambda^{i} z\right)}{2 n}
$$

Então

$$
\widetilde{p}(z)=\frac{1}{2 n} \sum_{j=0}^{m}\left(1+\lambda^{j}+\lambda^{2 j}+. .+\lambda^{(2 n-1) j}\right) a_{j} z^{j} .
$$

Observe que caso $2 n \mid j$

$$
\left(1+\lambda^{j}+\lambda^{2 j}+. .+\lambda^{(2 n-1) j}\right)=2 n,
$$

caso contrário

$$
\left(1+\lambda^{j}+\lambda^{2 j}+. .+\lambda^{(2 n-1) j}\right)=\frac{1-\lambda^{2 n j}}{1-\lambda^{j}}=0 .
$$

Então

$$
\widetilde{p}(z)=\sum_{2 n \mid j} a_{j} z^{j}
$$


Assim de (2.1)

$$
\begin{aligned}
|\omega(z)-\widetilde{p}(z)| & =\left|\sum_{i=0}^{2 n-1} \frac{\omega\left(\lambda^{i} z\right)}{2 n}-\sum_{i=0}^{2 n-1} \frac{p\left(\lambda^{i} z\right)}{2 n}\right| \\
& \leq \sum_{i=0}^{2 n-1} \frac{1}{2 n}\left|\omega\left(\lambda^{i} z\right)-p\left(\lambda^{i} z\right)\right| \\
& <\epsilon
\end{aligned}
$$

\subsection{O operador $L$}

Seja $I$ um intervalo limitado da reta real. Denote por $D_{a}(I)$ o conjunto a-estádio do intervalo $I$ de pontos no plano complexo cuja distancia ao intervalo $I$ é menor que $a>$ 0 . Tome $\epsilon>0$ suficientemente pequeno e considere o conjunto $D_{\epsilon}([0,1])=\{z \in \mathbb{C}$ : $\operatorname{dist}(I, z)<\epsilon\}$.

No que segue até o final do capítulo vamos a fixar o expoente crítico $\alpha=2 n$, com $n \in \mathbb{N} \backslash\{0\}$. Considere uma aplicação unimodal definida como uma aplicação $f=\phi \circ q_{t}$ : $[-1,1] \rightarrow[-1,1]$, com $t \in(0,1)$, onde $q_{t}:[-1,1] \rightarrow[-1,1]$, é

$$
q_{t}(x)=-2 t x^{\alpha}+2 t-1,
$$

com expoente crítico $\alpha$, e $\phi$ é algum difeomorfismo $C^{1}$, que preserva orientação, de $[-1,1]$. Denote $A_{t}(x)=-2 t x+2 t-1$. Então podemos escrever $f=\left(\phi \circ A_{t}\right)\left(x^{\alpha}\right)$. Nós sabemos que a aplicação $\tau: z \mapsto z^{\alpha}$ é holomorfa no plano complexo sem o zero, e a aplicação

$$
A_{t}: z \mapsto-2 t z+2 t-1
$$

onde $t \in D_{\epsilon}([0,1])$, é holomorfa em todo o plano complexo. Note que $q_{t}=A_{t} \circ \tau$.

Seja $V \subset \mathbb{C}$ um conjunto aberto contendo o intervalo $[-1,1]$. Escolha $\widetilde{V}$ um conjunto aberto contendo o intervalo $[-1,1]$ compactamente contido em $V$ tal que $A_{t} \circ \tau(\widetilde{V})$ esta compactamente contido em $V$. 
Definição 2.3 Definamos o operador composição analítico complexo

$$
L: \mathcal{A}_{V} \times D_{\epsilon}([0,1]) \rightarrow \mathcal{U}_{\widetilde{V}}
$$

como $L(\phi, t)(z)=\phi \circ q_{t}(z)$, para $z$ em $\overline{\widetilde{V}}$.

Observação 2.4 É fácil ver que se $(\phi, t) \in \mathcal{U}$ então

$$
L \circ \widetilde{\mathcal{R}}(\phi, t)=\mathcal{R} \circ L(\phi, t)
$$

Proposição 2.5 (Injetividade de L) Seja $\alpha>1$. Se temos $\phi, \widetilde{\phi} \in \mathcal{A}_{V}$ e t, $\tilde{t} \in \mathbb{C} \backslash\{0\}$ tal que

$$
\phi \circ q_{t}=\widetilde{\phi} \circ q_{\tilde{t}}
$$

sobre $[-1,1]$, ent $\tilde{a} o \phi=\widetilde{\phi}$ e $t=\widetilde{t}$.

Demonstração. Suponhamos que

$$
\phi \circ q_{t}=\widetilde{\phi} \circ q_{\tilde{t}}
$$

sobre $[-1,1]$. Então para todo $y \in[0,1]$ obtemos

$$
A_{t}(y)=\phi^{-1} \circ \widetilde{\phi} \circ A_{\widetilde{t}}(y) .
$$

Tome

$$
y=\frac{-x+2 \widetilde{t}-1}{2 \widetilde{t}}
$$

onde $x \in[-1,1]$. É fácil ver que

$$
\frac{t}{\widetilde{t}} x+\frac{t}{\widetilde{t}}-1=\phi^{-1} \circ \widetilde{\phi}(x) .
$$

Desde que sabemos que $\phi^{-1} \circ \widetilde{\phi}(1)=1$ e $\phi^{-1} \circ \widetilde{\phi}(-1)=-1$ então $t=\widetilde{t}$ e $\phi^{-1} \circ \widetilde{\phi}=i d$. 


\subsection{O diferencial de $L$ e suas propriedades}

Pela definição de $L$ para cada $(\omega, v)$ em $T_{\phi} \mathcal{A}_{V} \times \mathbb{C}$ temos

$$
\begin{aligned}
\mathcal{D} L(\phi, t)(\omega, 0) & =\left.\frac{d}{d u}\left[(\phi+u \omega) \circ q_{t}\right]\right|_{u=0} \\
& =\left.\frac{d}{d u}\left[\phi \circ q_{t}+u\left(\omega \circ q_{t}\right)\right]\right|_{u=0} \\
& =\omega \circ q_{t}
\end{aligned}
$$

e pela regra da cadeia

$$
\begin{aligned}
\mathcal{D} L(\phi, t)(0, v) & =\left.\frac{d}{d u}\left[\phi \circ q_{t+u v}\right]\right|_{u=0} \\
& =\left.D \phi\left(q_{t}\right) \cdot \frac{d}{d u}\left(q_{t+u v}\right)\right|_{u=0}
\end{aligned}
$$

onde

$$
\left.\frac{d}{d u}\left(q_{t+u v}(x)\right)\right|_{u=0}=2 v\left(1-x^{2 n}\right) .
$$

Assim a derivada de $L$ em $(\phi, t) \in \mathcal{A}_{V} \times D_{\epsilon}([0,1])$ é o operador analítico

$$
\mathcal{D} L(\phi, t): \mathcal{F}_{V} \times \mathbb{C} \rightarrow \mathcal{T}_{\widetilde{V}}
$$

dado por

$$
\mathcal{D} L(\phi, t)(\omega, v)(x)=\omega \circ q_{t}(x)+D \phi\left(q_{t}(x)\right) .2 v\left(1-x^{2 n}\right)
$$

para todo $x$ em $\widetilde{V}$.

Nas seguintes proposições provaremos algumas propriedades do diferencial $D L$.

Proposição 2.6 Seja $(\phi, t) \in \mathcal{A}_{V} \times(0,1]$. O operador $\mathcal{D} L(\phi, t)$ é injetivo.

Demonstração. Suponha que $\mathcal{D} L(\phi, t)(\omega, v)(z)=0$, para todo $z \in \widetilde{V}$. Então de $(2.3)$ temos

$$
\begin{aligned}
\omega \circ q_{t}(z) & =-D \phi\left(q_{t}(z)\right) \cdot 2 v\left(1-z^{2 n}\right) \\
\omega \circ q_{t}(z) & =-D \phi\left(q_{t}(z)\right) \cdot \frac{v}{t}\left(q_{t}(z)+1\right)
\end{aligned}
$$


De (2.5) temos que para qualquer $y \in\left[-1, q_{t}(0)\right] \subset[-1,1]$

$$
\omega(y)=-D \phi(y) \cdot \frac{v}{t}(y+1),
$$

Desde que $\omega(y)$ e $D \phi(y) \cdot \frac{v}{t}(y+1)$, são analíticos sobre $[-1,1]$ temos que a Eq. (2.6) se cumpre para qualquer $y \in[-1,1]$. Se tomarmos $y=1$ obtemos

$$
\omega(1)=-D \phi(1) \cdot \frac{v}{t}(2)
$$

e desde que $\omega(1)=0$ e $D \phi(1) \neq 0$ isto implica que $v=0$. Por outro lado a condição $v=0$ em (2.6) e a analiticidade de $\omega$ sobre $V$ implica $\omega(y)=0$, para todo $y \in V$. Assim $\mathcal{D} L(\phi, t)$ é injetivo.

Lema 2.7 Seja $(\phi, t) \in \mathcal{A}_{V} \times(0,1]$. A imagem do operador

$$
\mathcal{D} L(\phi, t): \mathcal{F}_{V} \times \mathbb{C} \rightarrow \mathcal{T}_{\widetilde{V}}
$$

é densa.

Demonstração. Pelo Lema 2.2 o conjunto de campos de vetores polinomiais é denso em $\mathcal{T}_{\widetilde{V}}$. Assim é suficiente mostrarmos que para todo polinômio $\widetilde{\omega} \in \mathcal{T}_{\widetilde{V}}$, existe $(\omega, v) \in \mathcal{F}_{V} \times \mathbb{C}$ tal que

$$
\mathcal{D} L(\phi, t)(\omega, v)=\widetilde{\omega} .
$$

Desde que $\widetilde{\omega}$ é da forma $\widetilde{\omega}=\psi\left(x^{2 n}\right)$, onde $\psi$ é um campo de vetores polinomial numa vizinhança de $[0,1]$, podemos representa-lo como $\widetilde{\omega}=\beta \circ q_{t}$, onde $\beta=\psi \circ A_{t}^{-1}$ é um campo de vetores polinomial. Tome

$$
\omega(y)=\beta(y)-D \phi(y) \frac{\beta(1)}{2 D \phi(1)}(1+y)
$$

e

$$
v=\frac{\beta(1) t}{2 D \phi(1)}
$$

Lembremos que um operador linear contínuo $T$ de um espaço de Banach $E$ sobre o espaço de Banach $F$ é compacto se, para cada sequência limitada $\left\{x_{n}\right\}$ em $E$, a sequência $\left\{T x_{n}\right\}$ contem uma subsequência convergente para algum limite em $F$. 
Lema 2.8 Seja $(\phi, t) \in \mathcal{A}_{V} \times D_{\epsilon}([0,1])$. O operador

$$
\mathcal{D} L(\phi, t): \mathcal{F}_{V} \times \mathbb{C} \rightarrow \mathcal{T}_{\widetilde{V}}
$$

é compacto.

Demonstração. Seja $\left\{\left(\omega_{i}, v_{i}\right)\right\} \subset \mathcal{F}_{V} \times \mathbb{C}$ uma sequência limitada, isto é existe uma constante $B>0$ tal que

$$
\left|\omega_{i}\right|_{V},\left|v_{i}\right|<B
$$

para todo $i>0$. Pela definição de $L$, temos que $\left\{\mathcal{D} L(\phi, t)\left(\omega_{i}, v_{i}\right)\right\}$ é uma sequência de campos de vetores analíticos sobre $\widetilde{V}$. Nós tínhamos tomado $\widetilde{V}$ compactamente contido em $V$ tal que $q_{t}(\widetilde{V})$ esta compactamente contido em $V$. Agora tomemos um subconjunto aberto $U \ni \widetilde{V}$ compactamente contido em $V$ tal que $q_{t}(U)$ esta compactamente contido em $V$. Então

$$
\left|\omega_{i}\right|_{V}=\sup _{x \in V}\left|\omega_{i}(x)\right| \geq \sup _{x \in q_{t}(U)}\left|\omega_{i}(x)\right|=\sup _{y \in U}\left|\omega_{i} \circ q_{t}(x)\right|=\left|\omega_{i} \circ q_{t}\right|_{U} .
$$

Assim $\omega_{i} \circ q_{t}$ é limitado sobre $U$ por $B$. Desde que $D \phi$ é também limitado em $q_{t}(U) \subset V$ para 2.3 existe $C>0$ tal que

$$
\left|\mathcal{D} L(\phi, t)\left(\omega_{i}, v_{i}\right)\right|_{U}<C
$$

para todo $i>0$. Desde que a sequência de aplicações analíticas em $U$ está limitada por uma constante que não depende de $i>0$ esta forma uma família normal em $U$, toda sequência $\left\{\mathcal{D} L(\phi, t)\left(\omega_{i}, v_{i}\right)\right\}$ contém uma subsequência convergente sobre $\widetilde{V}$. 


\section{Capítulo 3}

\section{Operadores de Renormalização Complexos}

Na Seção 3.1, fixando o expoente crítico $\alpha=2 n$ onde $n \in \mathbb{N}$, vamos a considerar o operador de renormalização $\mathcal{R}_{\alpha}$ definido no Capítulo 1 como um operador atuando sobre o espaço de funções holomorfas. Já na Seção 3.2, para o expoente crítico $\alpha>1$, definimos o novo operador de renormalização complexo $\widetilde{\mathcal{R}}_{\alpha}$ agindo no espaço de pares onde a primeira componente é uma aplicação holomorfa e a segunda é um número real. Na última seção, mostraremos que quando $\alpha>1$ é par, o espectro de $D \mathcal{R}_{\alpha}$ e $D \widetilde{\mathcal{R}}_{\alpha}$ coincidem nos respectivos pontos fixos dos operadores de renormalização $\mathcal{R}_{\alpha}$ e $\widetilde{\mathcal{R}}_{\alpha}$.

\subsection{Operador complexo $\mathcal{R}_{\alpha}$}

Baseado em métodos reais Marco Martens [17] provou a existência de pontos fixos do operador de renormalização $\mathcal{R}_{\alpha}$, de qualquer tipo combinatorial, agindo no espaço de aplicações unimodais suaves com expoente crítico $\alpha>1$. Da definição do operador de 
renormalização $\mathcal{R}_{\alpha}$ temos que seu ponto fixo, denotado por $f^{*}$, satisfaz

$$
f^{*}(z)=\frac{1}{p} f^{* q}(p z)
$$

para algum $p \in(-1,1)$ tal que $\left(f^{*}\right)^{q}(p)=p$.

Dada uma função analítica $f: V \rightarrow \mathbb{C}$, onde $V$ é um conjunto aberto, defina o conjunto aberto

$$
\mathcal{D}_{V}^{n}(f):=\bigcap_{i=0}^{n-1} f^{-i} V .
$$

Dado um subconjunto $V \subset \mathbb{C}$ e $\lambda \in \mathbb{C}$, denotamos $\lambda V:=\{\lambda x: x \in V\}$.

Como uma conseqüência da cota complexa a priori de Sullivan [6], fixando o expoente crítico $\alpha=2 n$ onde $n \in \mathbb{N}$, para todo $\varepsilon>0$ suficientemente pequeno existe $N_{1}=N_{1}(\varepsilon)>$ 0 suficientemente grande tal que os domínios $D_{\varepsilon / 2} \Subset D_{\varepsilon} \Subset D_{2 \varepsilon}$, satisfazem:

- $f^{*}$ tem extensão continua a $\bar{D}_{\varepsilon}$ o qual é holomorfo em $D_{\varepsilon}$, e tem um único ponto crítico em $D_{\varepsilon}$.

- Nós temos

$$
p^{N_{1}} D_{2 \varepsilon} \Subset \mathcal{D}_{D_{\varepsilon / 2}}^{q^{N_{1}}}\left(f^{*}\right),
$$

em outras palavras, podemos iterar $f^{*}: D_{\varepsilon / 2} \rightarrow \mathbb{C}$ ao menos $q^{N_{1}}$ vezes sobre o domínio $p^{N_{1}} \bar{D}_{2 \varepsilon}$.

Agora podemos definir o operador de renormalização complexo atuando sobre funções holomorfas suficientemente próximas a $f^{*}$. Pelo Lema B.1 podemos escolher $\gamma_{1}=\gamma_{1}\left(\varepsilon, N_{1}\right)>$ 0 suficientemente pequeno tal que para cada $f$ na bola de centro $f^{*}$ e raio $\gamma_{1}$ denotada por

$$
B\left(f^{*}, \gamma_{1}\right):=\left\{f \in \mathcal{U}_{D_{\varepsilon / 2}},\left|f-f^{*}\right|<\gamma_{1}\right\}
$$

o seguinte se cumpre:

- Existe uma continuação analítica $p_{f}$ do ponto periódico $p$ de $f^{*}$, isto é $f^{q}\left(p_{f}\right)=p_{f}$ e $p_{f} \sim p$.

- Tem-se $p_{f} D_{2 \varepsilon} \Subset D_{D_{\varepsilon / 2}}^{q^{N_{1}}}(f)$. 
Definimos o operador analítico complexo $\widehat{\mathcal{R}}_{2 n}: B\left(f^{*}, \gamma_{1}\right) \rightarrow \mathcal{U}_{D_{2 \varepsilon}}$ como

$$
\widehat{\mathcal{R}}_{2 n}(f)(z):=\frac{1}{p_{f}} f^{q^{N_{1}}}\left(p_{f} z\right) .
$$

Assim definimos a extensão analítica complexa do operador de renormalização como

$$
\mathcal{R}_{2 n}:=i \circ \widehat{\mathcal{R}}_{2 n}
$$

Observação 3.1 Note que temos liberdade para a escolha de $\varepsilon>0$ e $N_{1}>0$, e $\gamma_{1}>0$. Na Seção 3.3 faremos uma escolha conveniente para estes.

Segue do seguinte lema que $\mathcal{R}_{2 n}$ é um operador compacto.

Lema 3.2 A inclusão natural $i: \mathcal{U}_{D_{2 \varepsilon}} \rightarrow \mathcal{U}_{D_{\varepsilon / 2}}$ é um operador compacto entre os espaços de Banach.

Demonstração. Segue-se do Teorema de Montel.

Edson de Faria, W. de Melo and A. Pinto [8], com a ajuda das cotas reais e complexas a priori de Sullivan [6] e o resultado de hiperbolicidade de Lyubich [16] (veja também [5]), provaram a hiperbolicidade do ponto fixo do operador de renormalização com respeito a um iterado do operador de renormalização atuando sobre o espaço $\mathcal{U}_{D_{\varepsilon / 2}}$ para algum $\varepsilon>0$. Mais precisamente o Teorema 2.4 em [8] afirma que:

Teorema 3.3 Para $\varepsilon>0$ suficientemente pequeno, existe um número positivo $N_{1}=$ $N_{1}(\varepsilon)>0$ e $\gamma_{1}>0$ com a seguinte propriedade. O operador real analítico $\mathcal{R}_{2 n}$ : $B\left(f^{*}, \gamma_{1}\right) \rightarrow \mathcal{U}_{D_{\varepsilon / 2}}$, definido pela Eq. (3.1), tem um ponto fixo hiperbólico $f^{*}=\phi^{*} \circ q_{t^{*}} \in$ $B\left(f^{*}, \gamma_{1}\right)$ com variedade estável de codimensão um.

\subsection{Operador complexo $\widetilde{\mathcal{R}}_{\alpha}$}

Em [17, Teorema 2.2] Marco Martens prova que o novo operador de renormalização $\widetilde{\mathcal{R}}_{\alpha}$, com expoente crítico $\alpha>1$, o qual ele chama de operador de renormalização dinâmico, 
tem pontos fixos de qualquer tipo combinatorial, cuja primeira componente são difeomorfismos $\mathcal{C}^{2}$ em $[-1,1]$. Utilizando os métodos da demonstração do Teorema 1.17 e métodos em Martens é possível mostrar que de fato as primeiras componentes de tais pontos fixos de $\widetilde{\mathcal{R}}_{\alpha}$ são analíticos e univalentes numa vizinhança do intervalo $[-1,1]$. Fixemos uma combinatória $\sigma$ e denotemos por $\left(\phi^{*}, t^{*}\right)$ um ponto fixo analítico do operador de renormalização $\widetilde{\mathcal{R}}_{\alpha}$ com combinatória $\sigma$.

Pelo Teorema 1.17 temos uma cota complexa a priori para a primeira componente do novo operador de renormalização $\widetilde{\mathcal{R}}_{\alpha}$, onde o expoente crítico $\alpha>1$ é fixo. Assim seja $\delta<\delta_{0}$ pequeno e $\widetilde{N}_{1}=\widetilde{N}_{1}(\delta)>0$ como no Teorema 1.17. E pelo Corolário 1.18 temos que $\left(\phi^{*}, t^{*}\right)=\widetilde{\mathcal{R}}_{\alpha}^{\widetilde{N}_{1}}\left(\phi^{*}, t^{*}\right)$ é um par cuja primeira componente é univalente em $D_{2 \delta}$.

Fixemos um par $(\phi, t) \widetilde{N}_{1}$-vezes renormalizável, com expoente crítico $\widetilde{\alpha}$ suficientemente proximo de $\alpha$, e seja $\mathcal{I}^{\widetilde{N}_{1}}=\left\{I_{1}^{\widetilde{N}_{1}}, I_{2}^{\widetilde{N}_{1}}, \ldots, I_{\widetilde{N}_{1}}^{\widetilde{N}_{1}}\right\}$ o ciclo correspondente à $\widetilde{N}_{1}$-ésima renormalização. Nós sabemos que se $(\phi, t)$ satisfaz as hipóteses do Teorema 1.17, as aplicações $\phi_{0}^{\widetilde{N}_{1}}=Z_{\phi^{-1}\left(I_{1}^{\widetilde{N}_{1}}\right)}(\phi), q_{i}^{\widetilde{N}_{1}}=Z_{I_{i}^{\widetilde{N}_{1}}}\left(q_{t}\right)$ e $\phi_{i}^{\widetilde{N}_{1}}=Z_{q_{t}\left(I_{i}^{\widetilde{N}_{1}}\right)}(\phi)$, tem extensão univalente em domínios complexos de modo que a composição

$$
\left(\phi_{q_{\widetilde{N}_{1}}-1}^{\widetilde{N}_{1}} \circ q_{q_{\widetilde{N}_{1}-1}^{\widetilde{N}_{1}}}^{\widetilde{N}_{1}}\right) \circ \ldots \circ\left(\phi_{2}^{\widetilde{N}_{1}} \circ q_{2}^{\widetilde{N}_{1}}\right) \circ\left(\phi_{1}^{\widetilde{N}_{1}} \circ q_{1}^{\widetilde{N}_{1}}\right) \circ \phi_{0}^{\widetilde{N}_{1}}
$$

está definida em $D_{2 \delta}$. Logo pelo Lema B.1 podemos escolher $\widetilde{\gamma}_{1}>0$ suficientemente pequeno tal que o operador $\widetilde{\mathcal{R}}_{\alpha}^{\widetilde{N}_{1}}$ tem uma extensão para a bola

$$
\left.\widetilde{B}\left(\left(\phi^{*}, t^{*}\right), \gamma\right):=\{(\phi, t)) \in \mathcal{A}_{D_{\delta / 2}} \times \mathbb{C},\left|(\phi, t)-\left(\phi^{*}, t^{*}\right)\right|<\widetilde{\gamma}_{1}\right\}
$$

como uma transformação $\widetilde{\widetilde{\mathcal{R}}}_{\alpha}: \widetilde{B}\left(\left(\phi^{*}, t^{*}\right), \widetilde{\gamma}_{1}\right) \rightarrow \mathcal{A}_{D_{2 \delta}} \times \mathbb{C}$, definida pela expressão

$$
\widetilde{\widetilde{\mathcal{R}}}_{\alpha}(\phi, t)=\left(\left(\phi_{q_{\tilde{N}_{1}}}^{\widetilde{N}_{1}} \circ q_{q_{\widetilde{N}_{1}}}^{\widetilde{N}_{1}}\right) \circ \ldots \circ\left(\phi_{2}^{\widetilde{N}_{1}} \circ q_{2}^{N_{2}}\right) \circ\left(\phi_{1}^{\widetilde{N}_{1}} \circ q_{1}^{\widetilde{N}_{1}}\right) \circ \phi_{0}^{\widetilde{N}_{1}}, t_{\widetilde{N}_{1}}\right)
$$

onde

$$
t_{\widetilde{N}_{1}}=\frac{\left|q_{t}\left(I_{0}^{\widetilde{N}_{1}}\right)\right|}{\left|\phi^{-1}\left(I_{1}^{\widetilde{N}_{1}}\right)\right|} .
$$

A inclusão natural $j: \mathcal{A}_{D_{2 \delta}} \times \mathbb{C} \rightarrow \mathcal{A}_{D_{\delta / 2}} \times \mathbb{C}$ é um operador compacto entre os espaços de Banach. Então definimos o operador de renormalização complexo por

$$
\widetilde{\mathcal{R}}_{\alpha}=j \circ \widetilde{\widetilde{\mathcal{R}}}_{\alpha}
$$


Na seguinte proposição provamos que o novo operador de renormalização $\widetilde{\mathcal{R}}_{\alpha}$ é analítico complexo.

Proposição 3.4 A transformação $(\phi, t, \alpha) \rightarrow \widetilde{\mathcal{R}}_{\alpha}(\phi, t)$ é analítica complexa nas variáveis $(\phi, t)$ e analítico real na variável $\alpha$.

Demonstração. Seja $\left(\phi^{*}, t^{*}\right)$ o ponto fixo do operador $\widetilde{\mathcal{R}}_{\alpha}$. Seja $p=p(\phi, t) \in(0,1)$ o ponto periódico da aplicação $\phi \circ q_{t}$, com período $q \geq 2$, isto é $\left(\phi \circ q_{t}\right)^{q}(p)=p$, que é a continuação analítica do ponto periódico na fronteira do intervalo central do ciclo de renormalização de $\phi^{*} \circ q_{t^{*}}$. E seja $b=b(\phi, t) \in(p, 1)$ satisfazendo $\left(\phi \circ q_{t}\right)^{q}(b)=-p$. Denotemos por $\phi(\widetilde{p})=p$ e $\phi(\widetilde{b})=b$. Suponhamos que $\widetilde{N}_{1}=1$ para simplificar um pouco a notação. Assim nós temos que

$$
\widetilde{\mathcal{R}}_{\alpha}(\phi, t)=(F((\phi, t), \alpha), \rho((\phi, t), \alpha))
$$

onde

$$
\begin{gathered}
F((\phi, t), \alpha)=\frac{1}{p}(\underbrace{\phi \circ q_{t} \circ \cdots \circ q_{t} \circ \phi}_{2 q-1 \text { termos }} \circ \gamma_{2}^{-1}(x)), \\
\left.\left.\gamma_{2}^{-1}(x)\right)=\frac{\widetilde{b}-\widetilde{p}}{2} \cdot(x+1)+\widetilde{p}, \text { e } \rho((\phi, t), \alpha)\right)=\frac{2 t-1-q_{t}(p)}{\widetilde{b}-q_{t}(p)}=\frac{2 t p^{\alpha}}{\widetilde{b}-\widetilde{p}} .
\end{gathered}
$$

Primeiro derivamos $F$ com respeito a $(\phi, t)$ e $\alpha$. Assim

$$
\begin{aligned}
\left(\mathcal{D}_{\phi} F \cdot \omega\right)(x)= & -\frac{1}{p}\left(\mathcal{D}_{\phi} p \cdot \omega\right)(x) F(x)+\frac{1}{p}\left[D(\underbrace{\phi \circ q_{t} \circ \cdots \phi \circ q_{t} \circ \phi}_{2 q-1})\left(\gamma_{2}^{-1}(x)\right)\left(\mathcal{D}_{\phi} \gamma_{2}^{-1} \cdot \omega\right)(x)\right. \\
& +\sum_{i=0}^{q-1} D(\underbrace{\phi \circ q_{t} \circ \cdots \phi \circ q_{t}}_{2 i})(\underbrace{\phi \circ q_{t} \circ \cdots \phi}_{2 q-1-2 i} \circ \gamma_{2}^{-1}(x)) \\
& \omega(\underbrace{\left.q_{t} \circ \cdots q_{t} \circ \phi \circ \gamma_{2}^{-1}(x)\right)}_{2(q-1)-2 i}], \\
\left(\mathcal{D}_{t} F \cdot v\right)(x)=- & \frac{1}{p}\left(\mathcal{D}_{t} p \cdot v\right)(x) F(x)+\frac{1}{p}\left[D(\underbrace{\phi \circ q_{t} \circ \cdots \phi \circ q_{t} \circ \phi}_{2 q-1})\left(\gamma_{2}^{-1}(x)\right)\left(\mathcal{D}_{t} \gamma_{2}^{-1} \cdot v\right)(x)\right. \\
+ & \sum_{i=1}^{q-1} D(\underbrace{\phi \circ q_{t} \circ \cdots \phi \circ q_{t}}_{2 q-1-2 i})(\underbrace{\phi \circ q_{t} \circ \cdots \phi}_{2 i} \circ \gamma_{2}^{-1}(x)) \\
& \left.\left(\mathcal{D}_{t} q_{t} \cdot v\right)(\underbrace{\phi \circ q_{t} \circ \cdots q_{t} \circ \phi}_{2 i-1} \circ \gamma_{2}^{-1}(x))\right],
\end{aligned}
$$


$\mathrm{e}$

$$
\begin{aligned}
\left(\mathcal{D}_{\alpha} F \cdot \tau\right)(x)= & -\frac{1}{p}\left(\mathcal{D}_{\alpha} p \cdot \tau\right)(x) F(x)+\frac{1}{p}\left[D(\underbrace{\phi \circ q_{t} \circ \cdots \phi \circ q_{t} \circ \phi}_{2 q-1})\left(\gamma_{2}^{-1}(x)\right)\left(\mathcal{D}_{\alpha} \gamma_{2}^{-1} \cdot \tau\right)(x)\right. \\
& +\sum_{i=1}^{q-1} D(\underbrace{\phi \circ q_{t} \circ \cdots \phi \circ q_{t}}_{2 q-1-2 i})(\underbrace{\phi \circ q_{t} \circ \cdots \phi}_{2 i} \circ \gamma_{2}^{-1}(x)) \\
& \left.\left(\mathcal{D}_{\alpha} q_{t} \cdot \tau\right)(\underbrace{\phi \circ q_{t} \circ \cdots q_{t} \circ \phi}_{2 i-1} \circ \gamma_{2}^{-1}(x))\right] .
\end{aligned}
$$

Observe que $\underbrace{\phi \circ q_{t} \circ \cdots q_{t} \circ \phi}_{2 i-1} \circ \gamma_{2}^{-1}(x)$, onde $x \in D_{\delta / 2}$ e $i=1, \cdots, q-1$, pertence ao domínio $S_{\widetilde{\alpha}}^{+}$ou $S_{\widetilde{\alpha}}^{-}$, os quais foram introduzidos na prova do Teorema 1.23. Sejam $q_{t}^{+}$e $q_{t}^{-}$como definidos na mesma prova. Quando $\underbrace{\phi \circ q_{t} \circ \cdots q_{t} \circ \phi}_{2 i-1} \circ \gamma_{2}^{-1}(x) \in S_{\widetilde{\alpha}}^{+}$obtemos $\left(\mathcal{D}_{t} q_{t} \cdot v\right)(\underbrace{\phi \circ q_{t} \circ \cdots q_{t} \circ \phi}_{2 i-1} \circ \gamma_{2}^{-1}(x))=-2 v \exp \left(\alpha \log (\underbrace{\phi \circ q_{t} \circ \cdots q_{t} \circ \phi}_{2 i-1} \circ \gamma_{2}^{-1}(x))\right)+2 v$, e

$$
\begin{aligned}
\left(\mathcal{D}_{\alpha} q_{t} \cdot \tau\right)(\underbrace{\phi \circ q_{t} \circ \cdots q_{t} \circ \phi}_{2 i-1} \circ \gamma_{2}^{-1}(x))= & -2 t \log (\underbrace{\phi \circ q_{t} \circ \cdots q_{t} \circ \phi}_{2 i-1} \circ \gamma_{2}^{-1}(x)) \\
& \exp \left(\alpha \log (\underbrace{\phi \circ q_{t} \circ \cdots q_{t} \circ \phi}_{2 i-1} \circ \gamma_{2}^{-1}(x))\right) \tau
\end{aligned}
$$

para $i=1, \cdots, q-1$. Aqui log é o ramo de logaritmo definido em $S_{\widetilde{\alpha}}^{+}$. Agora quando $\underbrace{\phi \circ q_{t} \circ \cdots q_{t} \circ \phi}_{2 i-1} \circ \gamma_{2}^{-1}(x) \in S_{\widetilde{\alpha}}^{-}$o cálculo das derivadas parciais é análogo. Da relação $\left(\phi \circ q_{t}\right)^{q}(p)=p$ derivando com respeito a $(\phi, t)$ e $\alpha$ obtemos

$$
\begin{aligned}
\left(\mathcal{D}_{\phi} p \cdot \omega\right)(x)= & \frac{1}{1-D(\underbrace{\phi \circ q_{t} \circ \cdots \phi \circ q_{t} \circ \phi \circ q_{t}}_{2 q})(p)} \\
& \sum_{i=0}^{q} D(\underbrace{\phi \circ q_{t} \circ \cdots \phi \circ q_{t}}_{2 i})(\underbrace{\phi \circ q_{t} \circ \cdots \phi \circ q_{t}}_{2 q-2 i}(p)) \omega(\underbrace{q_{t} \circ \cdots q_{t} \circ \phi \circ q_{t}}_{2 q-1-2 i}(p)),
\end{aligned}
$$




$$
\begin{aligned}
\left(\mathcal{D}_{t} p \cdot v\right)(x)= & \frac{1}{1-D(\underbrace{\phi \circ q_{t} \circ \cdots \phi \circ q_{t} \circ \phi \circ q_{t}}_{2 q})(p)} \\
& \sum_{i=0}^{q} D(\underbrace{\phi \circ q_{t} \circ \cdots \phi}_{2 q-1-2 i})(\underbrace{q_{t} \circ \cdots \phi \circ q_{t}}_{2 i+1}(p))\left(\mathcal{D}_{t} q_{t} \cdot v\right)(\underbrace{\phi \circ q_{t} \circ \cdots q_{t} \circ \phi \circ q_{t}}_{2 i}(p)),
\end{aligned}
$$

e

$$
\begin{aligned}
\left(\mathcal{D}_{\alpha} p \cdot \tau\right)(x)= & \frac{1}{1-D(\underbrace{\phi \circ q_{t} \circ \cdots \phi \circ q_{t} \circ \phi \circ q_{t}}_{2 q})(p)} \\
& \sum_{i=0}^{q} D(\underbrace{\phi \circ q_{t} \circ \cdots \phi}_{2 q-1-2 i})(\underbrace{q_{t} \circ \cdots \phi \circ q_{t}}_{2 i+1}(p))\left(\mathcal{D}_{\alpha} q_{t} \cdot \tau\right)(\underbrace{\phi \circ q_{t} \circ \cdots q_{t} \circ \phi \circ q_{t}}_{2 i}(p)) .
\end{aligned}
$$

As derivadas parciais para $b$ com respeito a $\phi, t$ e $\alpha$ são obtidas da seguinte relação

$$
\left(\phi \circ q_{t}\right)^{q}(b)=-p
$$

e elas podem ser obtidas de forma similar ao cálculo da derivadas parciais de $p$. Das relações $\phi(\widetilde{p})=p$ e $\phi(\widetilde{b})=b$ temos

$$
\left(\mathcal{D}_{(\phi, t)} \widetilde{p} \cdot(\omega, v)\right)(x)=\frac{\left(\mathcal{D}_{(\phi, t)} p \cdot(\omega, v)\right)(x)-\omega(\widetilde{p})}{D \phi(\widetilde{p})},
$$

$\mathrm{e}$

$$
\left(\mathcal{D}_{\alpha} \tilde{p} \cdot \tau\right)(x)=\frac{\left(\mathcal{D}_{\alpha} p \cdot \tau\right)(x)}{D \phi(\widetilde{p})}
$$

similarmente

$$
\left(\mathcal{D}_{(\phi, t)} \tilde{b} \cdot(\omega, v)\right)(x)=\frac{\left(\mathcal{D}_{(\phi, t)} b \cdot(\omega, v)\right)(x)-\omega(\widetilde{b})}{D \phi(\widetilde{b})},
$$

e

$$
\left(\mathcal{D}_{\alpha} \widetilde{b} \cdot \tau\right)(x)=\frac{\left(\mathcal{D}_{\alpha} b \cdot \tau\right)(x)}{D \phi(\widetilde{b})} .
$$

Logo das derivadas obtidas se pode obter facilmente as derivadas parciais de

$$
\left.\gamma_{2}^{-1}(x)\right)=\frac{\widetilde{b}-\widetilde{p}}{2} \cdot(x+1)+\widetilde{p}
$$

Por outro lado $\rho((\phi, t), \alpha)$ tem as seguintes derivadas parciais

$$
\left(\mathcal{D}_{\phi} \rho \cdot \omega\right)(x)=\frac{D q_{t}(p)\left(\mathcal{D}_{\phi} p \cdot \omega\right)(x)(\widetilde{b}-\widetilde{p})-2 t p^{\alpha}\left(\left(\mathcal{D}_{\phi} \widetilde{b} \cdot \omega\right)(x)-\left(\mathcal{D}_{\phi} \widetilde{p} \cdot \omega\right)(x)\right)}{(\widetilde{b}-\widetilde{p})^{2}},
$$


$\left(\mathcal{D}_{t} \rho \cdot v\right)(x)=\frac{2 v p^{\alpha}}{\widetilde{b}-\widetilde{p}}-\frac{D q_{t}(p) \mathcal{D}_{t} p \cdot(v)(x)(\widetilde{b}-\widetilde{p})-2 t p^{\alpha}\left(\left(\mathcal{D}_{t} \widetilde{b} \cdot v\right)(x)-\left(\mathcal{D}_{\phi} \widetilde{p} \cdot v\right)(x)\right)}{(\widetilde{b}-\widetilde{p})^{2}}$,

e

$\left(\mathcal{D}_{\alpha} \rho \cdot \tau\right)(x)=\frac{\left(2 t p^{\alpha} \tau \log p+D q_{t}(p)\left(\mathcal{D}_{\alpha} p \cdot \tau\right)(x)\right)(\widetilde{b}-\widetilde{p})-2 t p^{\alpha}\left(\left(\mathcal{D}_{\alpha} \widetilde{b} \cdot \tau\right)(x)-\left(\mathcal{D}_{\alpha} \widetilde{p} \cdot \tau\right)(x)\right)}{(\widetilde{b}-\widetilde{p})^{2}}$.

Quando o ponto periódico $p \in(-1,0)$ temos que $b \in(-1, p)$ e o cálculo das derivadas parciais seguem de forma similar.

\subsection{Relação entre os operadores complexos $\mathcal{R}_{2 n}$ e $\widetilde{\mathcal{R}}_{2 n}$}

Agora considere o expoente crítico $\alpha=2 n$, onde $n \in \mathbb{N}$. Com as notações da seção anterior, escolha $\delta>0, \widetilde{N}_{1}$ e $\widetilde{\gamma}_{1}$ como na Seção 3.2. Seja $\varepsilon_{0}$ tal que

$$
q_{t^{*}}\left(\bar{D}_{2 \varepsilon_{0}}\right) \subseteq D_{\delta / 2}
$$

E escolha $\varepsilon<\varepsilon_{0}, N_{1}$ e $\gamma_{1}>0$ como no Teorema 3.3. Logo escolhemos $N=N_{1} \cdot \widetilde{N_{1}}$ e consideramos este na definição dos operadores $\mathcal{R}_{2 n}$ e $\widetilde{\mathcal{R}}_{2 n}$ das seções anteriores.

Desde que $\widetilde{\widetilde{\mathcal{R}}}_{2 n}$ é analítico existe $\widetilde{C}>1$ tal que

$$
\widetilde{\widetilde{\mathcal{R}}}_{2 n}\left(\widetilde{B}_{\delta / 2}\left(\left(\phi^{*}, t^{*}\right), \widetilde{\gamma}\right)\right) \subseteq \widetilde{B}_{\delta}\left(\left(\phi^{*}, t^{*}\right), \widetilde{C} \cdot \widetilde{\gamma}\right)
$$

para todo $\widetilde{\gamma}<\widetilde{\gamma}_{1}$. Logo existe $\widetilde{\gamma}_{2}$ tal que se

$$
\left|(\phi, t)-\left(\phi^{*}, t^{*}\right)\right|<\widetilde{C} \cdot \widetilde{\gamma}_{2}
$$

então $\phi \circ q_{t}$ esta definida em $\bar{D}_{2 \varepsilon}$. Em particular para cada $\widetilde{\gamma} \leq \widetilde{\gamma}_{2}$ temos definidos os seguintes operadores de composição

$$
L: \widetilde{B}_{\delta / 2}\left(\left(\phi^{*}, t^{*}\right), \widetilde{\gamma}\right) \rightarrow \mathcal{U}_{2 \varepsilon}
$$

e

$$
L: \widetilde{B}_{\delta / 2}\left(\left(\phi^{*}, t^{*}\right), \widetilde{C} \cdot \widetilde{\gamma}\right) \rightarrow \mathcal{U}_{2 \varepsilon}
$$


Seja $\widetilde{\gamma}_{3}>0$ tal que se $\widetilde{\gamma} \leq \widetilde{\gamma}_{3}$ então

$$
L\left(\widetilde{B}_{\delta / 2}\left(\left(\phi^{*}, t^{*}\right), \widetilde{\gamma}\right)\right) \subseteq B_{2 \varepsilon}\left(\phi^{*} \circ q_{t^{*}}, \gamma_{1}\right) .
$$

Seja $\widetilde{\gamma} \leq \min \left\{\widetilde{\gamma}_{1}, \widetilde{\gamma}_{2}, \widetilde{\gamma}_{3}\right\}$. Logo observarmos que as seguintes composições são possíveis

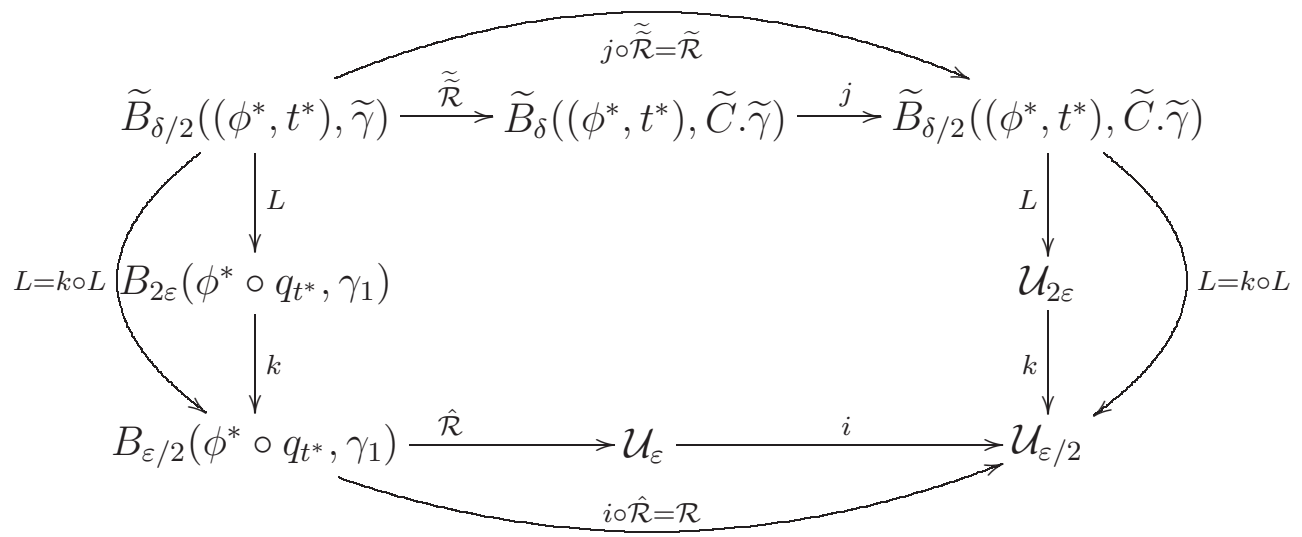

Em particular temos que

$$
\mathcal{R} \circ L=L \circ \widetilde{\mathcal{R}}
$$

Já para os respectivos espaços tangentes temos as seguintes composições:

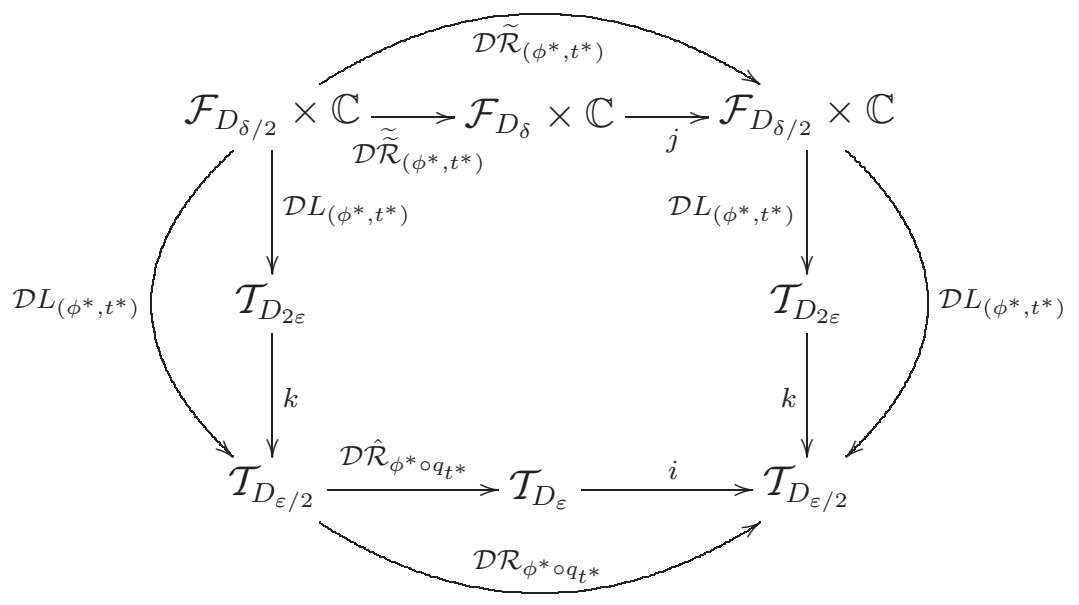

E podemos observar que

$$
\mathcal{D} \mathcal{R}_{L\left(\phi^{*}, t^{*}\right)} \cdot \mathcal{D} L_{\left(\phi^{*}, t^{*}\right)}=\mathcal{D} L_{\left(\phi^{*}, t^{*}\right)} \cdot \mathcal{D} \widetilde{\mathcal{R}}_{\left(\phi^{*}, t^{*}\right)} .
$$


Note que, pela Observação 2.4, $L\left(\phi^{*}, t^{*}\right)$ é ponto fixo do operador $\mathcal{R}$. Assim uma importante relação entre os operadores $\mathcal{R}$ e $\widetilde{\mathcal{R}}$ é o seguinte resultado.

Proposição 3.5 Seja $\left(\phi^{*}, t^{*}\right) \in \mathcal{A}_{D_{\delta / 2}} \times(0,1]$ ponto fixo do operador $\widetilde{\mathcal{R}}$ e $L\left(\phi^{*}, t^{*}\right)$ o correspondente ponto fixo de $\mathcal{R}$. Então

$$
\sigma\left(\mathcal{D} \widetilde{\mathcal{R}}_{\left(\phi^{*}, t^{*}\right)}\right)=\sigma\left(\mathcal{D} \mathcal{R}_{L\left(\phi^{*}, t^{*}\right)}\right)
$$

Demonstração. Denotemos por $f_{*}=\left(\phi^{*}, t^{*}\right)$. Seja $\lambda \neq 0$ um autovalor de $\mathcal{D} \widetilde{\mathcal{R}}_{f_{*}}$ com autovetor $v \neq 0$, isto é

$$
\mathcal{D} \widetilde{\mathcal{R}}_{f_{*}} v=\lambda v
$$

Pela relação de composição sobre os espaços tangentes mostrada acima temos

$$
\mathcal{D} \mathcal{R}_{L\left(f_{*}\right)} \cdot \mathcal{D} L_{f_{*}}=\mathcal{D} L_{f_{*}} \cdot \mathcal{D} \widetilde{\mathcal{R}}_{f_{*}}
$$

Então

$$
\begin{aligned}
\mathcal{D} \mathcal{R}_{L\left(f_{*}\right)} \cdot \mathcal{D} L_{f_{*}}(v) & =\mathcal{D} L_{f_{*}} \cdot \mathcal{D} \widetilde{\mathcal{R}}_{f_{*}}(v) \\
\mathcal{D} \mathcal{R}_{L\left(f_{*}\right)}\left(\mathcal{D} L_{f_{*}} v\right) & =\lambda\left(\mathcal{D} L_{f_{*}} v\right)
\end{aligned}
$$

assim $\lambda$ é autovalor de $\mathcal{D} \mathcal{R}_{L\left(f_{*}\right)} \operatorname{com} \mathcal{D} L_{f_{*}} v \neq 0$ da Proposição 2.6. Finalmente da compacidade dos operadores $\mathcal{D} \widetilde{\mathcal{R}}_{f_{*}}$ e $\mathcal{D} \mathcal{R}_{L\left(f_{*}\right)}$ e o Teorema A.3 segue-se que $\sigma\left(\mathcal{D} \widetilde{\mathcal{R}}_{f_{*}}\right) \subset$ $\sigma\left(\mathcal{D R}_{L\left(f_{*}\right)}\right)$.

Tome $\widetilde{\omega} \in \mathcal{T}_{D_{\varepsilon / 2}}$. Nós sabemos que $\widetilde{\omega}(x)=\psi\left(x^{2 n}\right)$, onde $\psi$ é holomorfo numa vizinhança de 0 . Como $\widetilde{\omega}(x)=\sum a_{i} x^{i}$, se $2 n \nmid i$ então $a_{i}=0$, assim $\psi(x)=\sum a_{2 n i} x^{i}$. O raio de convergência para $\psi$ denotado por $r_{\psi}$ não depende do raio de convergência de $\widetilde{\omega}$ denotado por $r_{\widetilde{\omega}}$ porque

$$
\begin{aligned}
r_{\psi} & =\frac{1}{\limsup _{i} \sqrt[i]{\left|a_{2 n i}\right|}} \\
& \geq\left(\frac{1}{\limsup _{i} \sqrt[i]{\left|a_{i}\right|}}\right)^{2 n} \\
& =r_{\widetilde{\omega}}^{2 n}
\end{aligned}
$$


Nós sabemos que $\psi$ é definido ao menos na bola de centro 0 e raio $\left(\frac{\varepsilon}{2}\right)^{2 n}$. Na verdade $\psi$ é definido num $\theta(2 n, \varepsilon)$-estádio do intervalo $[0,1]$ porque $\widetilde{\omega}$ é definido numa vizinhança do intervalo $[-1,1]$. Tomemos $\mu(2 n, \varepsilon, t)>0$ tal que a imagem do $\mu(2 n, \epsilon, t)$-estádio $D_{\mu(2 n, \varepsilon, t)}$, por $A_{t^{*}}^{-1}$, está contida no $\theta(2 n, \varepsilon)$-estádio do intervalo $[0,1]$. Agora defina

$$
F: \mathcal{T}_{D_{\varepsilon / 2}} \rightarrow \mathcal{F}_{D_{\mu(2 n, \varepsilon, t)}} \times \mathbb{C}
$$

por

$$
F(\widetilde{\omega})=(\omega, v)
$$

onde

$$
\omega(y)=\psi \circ A_{t^{*}}^{-1}(y)-D \phi^{\star}(y) \frac{\psi \circ A_{t^{*}}^{-1}(1)}{2 D \phi^{\star}(1)}(1+y)
$$

e

$$
v=\frac{\psi \circ A_{t^{*}}^{-1}(1) t^{*}}{2 D \phi^{\star}(1)}
$$

Seja $\lambda \neq 0$ um autovalor de $\mathcal{D} \mathcal{R}_{L\left(f_{*}\right)}$ com autovetor $w \neq 0$, isto é

$$
\mathcal{D} \mathcal{R}_{L\left(f_{*}\right)} w=\lambda w \text {. }
$$

Pela densidade da imagem do operador $\mathcal{D} L$ existe uma sequência $v_{s}$ tal que $\mathcal{D} L_{f_{*}} v_{s} \rightarrow w$. Como $F$ é um operador compacto então

$$
F \circ \mathcal{D} L_{f_{*}} v_{s}=i\left(v_{s}\right) \rightarrow F(w)
$$

Então

$$
\mathcal{D} L_{f_{*}} \cdot \mathcal{D} \widetilde{\mathcal{R}}_{f_{*}} i\left(v_{s}\right)=\mathcal{D} \mathcal{R}_{L\left(f_{*}\right)} \cdot \mathcal{D} L_{f_{*}} i\left(v_{s}\right)
$$

Tomando o limite quando $s \rightarrow \infty$ assim

$$
\mathcal{D} L_{f_{*}} \cdot \mathcal{D} \widetilde{\mathcal{R}}_{f_{*}} F(w)=\mathcal{D} \mathcal{R}_{L\left(f_{*}\right)} i(w)
$$

Aplicando $F$ na ultima equação temos

$$
\mathcal{D} \widetilde{\mathcal{R}}_{L\left(f_{*}\right)} F(w)=\lambda F i(w) .
$$


Finalmente diminuindo o domínio de $w$, se for necessário, que contém o intervalo $[-1,1]$, temos

$$
\mathcal{D} \widetilde{\mathcal{R}}_{L\left(f_{*}\right)} F(w)=\lambda F(w)
$$

Denotemos por $V_{\beta}$ e $\widetilde{V}_{\lambda}$ os autoespaços associados ao autovalor $\beta \in \sigma\left(\mathcal{D} \mathcal{R}_{L\left(\phi^{*}, t^{*}\right)}\right)$ e $\lambda \in \sigma\left(\mathcal{D} \widetilde{\mathcal{R}}_{\left(\phi^{*}, t^{*}\right)}\right)$ respectivamente, isto é

$$
V_{\beta}=N\left(\mathcal{D} \mathcal{R}_{L\left(\phi^{*}, t^{*}\right)}-\beta I d\right)=\left\{v:\left(\mathcal{D R}_{L\left(\phi^{*}, t^{*}\right)}-\beta I d\right) v=0\right\}
$$

e

$$
\widetilde{V}_{\lambda}=N\left(\mathcal{D} \widetilde{\mathcal{R}}_{\left(\phi^{*}, t^{*}\right)}-\lambda I d\right)=\left\{(\omega, t):\left(\mathcal{D} \widetilde{\mathcal{R}}_{\left(\phi^{*}, t^{*}\right)}-\lambda I d\right)(\omega, t)=0\right\} .
$$

Observe que estes autoespaços associados aos autovalores não dependem dos domínios de definição das aplicações que formam os autoespaços respectivos desde que ao aplicar o operador de renormalização $(\mathcal{R}$ ou $\widetilde{\mathcal{R}})$ estes terminam definidos num domínio maior.

Proposição 3.6 Seja $\left(\phi^{*}, t^{*}\right) \in \mathcal{A}_{D_{\delta / 2}} \times(0,1]$ o ponto fixo do operador $\widetilde{\mathcal{R}}$. Se $\lambda \in$ $\sigma\left(\mathcal{D} \widetilde{\mathcal{R}}_{\left(\phi^{*}, t^{*}\right)}\right) \backslash\{0\}$ então $\operatorname{dim} V_{\lambda}=\operatorname{dim} \widetilde{V}_{\lambda}$.

Demonstração. Pelo Teorema A.4 temos que $V_{\lambda}$ e $\widetilde{V}_{\lambda}$ são espaços finito dimensionais. Defina a aplicação continua

$$
T: \widetilde{V}_{\lambda} \rightarrow V_{\lambda}
$$

por $T(v)=\mathcal{D} L_{\left(\phi^{*}, t^{*}\right)}(v)$. Desde que $\mathcal{D} L_{\left(\phi^{*}, t^{*}\right)}$ é injetivo temos que $\operatorname{dim} \operatorname{Ker}(T)=0$. Então $\operatorname{dim} \widetilde{V}_{\lambda}=\operatorname{dim} \operatorname{Ker}(T)+\operatorname{dim} \operatorname{Im}(T) \leq \operatorname{dim} V_{\lambda}$. Também podemos definir a aplicação injetiva contínua

$$
\widetilde{T}: V_{\lambda} \rightarrow \widetilde{V}_{\lambda}
$$

por $\widetilde{T}(\widetilde{w})=F(\widetilde{w})$, onde $F$ é definida pela expressão 3.2. Então $\operatorname{dim} V_{\lambda} \leq \operatorname{dim} \widetilde{V}_{\lambda}$. 


\section{Capítulo 4}

\section{Hiperbolicidade para $\alpha \sim 2 n$}

\subsection{Hiperbolicidade do ponto fixo do operador $\widetilde{\mathcal{R}}_{2 n}$}

Seguindo com a notação da Seção 3.3 do Capítulo 3 temos o seguinte resultado de hiperbolicidade para o novo operador de renormalização $\widetilde{\mathcal{R}}_{2 n}$ que seria análogo ao Teorema 3.3 para este operador.

Teorema 4.1 Seja $\alpha=2 n$ o expoente crítico. Existe um número positivo $N$ tal que o operador $\widetilde{\mathcal{R}}_{2 n}$, como definido na Seção 3.3, tem um único ponto fixo unimodal $\left(\phi^{*}, t^{*}\right)$. Além disso $\left(\phi^{*}, t^{*}\right)$ é hiperbólico com variedade estável de codimensão um.

Demonstração. A existência do ponto fixo $\left(\phi^{*}, t^{*}\right)$ para o operador $\widetilde{\mathcal{R}}_{2 n}$ é garantida por Martens [17]. Desde que

$$
L \circ \widetilde{\mathcal{R}}_{2 n}\left(\phi^{*}, t^{*}\right)=\mathcal{R}_{2 n} \circ L\left(\phi^{*}, t^{*}\right)
$$

ver Observação 2.4, segue que $\phi^{*} \circ q_{t^{*}}$ é ponto fixo do operador $\mathcal{R}_{2 n}$. E por Sullivan [6] (garante unicidade do ponto fixo) e pelo Teorema 3.3 temos que o operador $\mathcal{R}_{2 n}$ tem ponto fixo hiperbólico $f^{*}=\phi^{*} \circ q_{t^{*}} \in B\left(f^{*}, \gamma_{1}\right)$ com variedade estável de codimensão um. Segue 
das Proposições 3.5 e 3.6 que o ponto fixo $\left(\phi^{*}, t^{*}\right)$ do operador $\widetilde{\mathcal{R}}_{2 n}$ é hiperbólico com variedade estável de codimensão um. A unicidade do ponto fixo segue da Proposição 2.5.

\subsection{Hiperbolicidade para $\alpha \sim 2 n$}

Agora pretendemos perturbar o expoente crítico $\alpha=2 n$. Mostraremos que se o expoente crítico $\alpha$ é proximo a $2 n, \alpha \sim 2 n$, o operador $\widetilde{\mathcal{R}}_{\alpha}$ tem um ponto fixo hiperbólico $\left(\phi_{\alpha}^{*}, t_{\alpha}^{*}\right)$ com variedade estável de codimensão um. Na seção anterior mostramos este resultado para $\alpha=2 n$. Fixemos uma combinatória $\sigma$. Denotamos por $\mathcal{H}_{\alpha}(C, \eta, M)$ o espaço dos pares $(\phi, t)$ satisfazendo

1. $\phi \in \mathcal{A}_{D_{\eta}}$ onde $\phi$ é univalente sobre $D_{\eta}$,

2. $\phi$ é real sobre a reta real,

3. $|\phi|_{\mathrm{C}^{3}([-1,1])} \leq C$,

4. $(\phi, t)$ é $M$-vezes renormalizável com combinatória $\sigma$ e expoente crítico $\alpha \sim 2 n$.

Nós podemos restabelecer o Teorema 1.15 na seguinte forma

Teorema 4.2 (Complex bounds) Existe $\delta_{0}$ e $C_{0}$ tal que o seguinte se satisfaz: para todo $C>0$ e $\eta>0$ existe $N_{0}=N_{0}(C, \eta)$ tal que se $(\phi, t) \in \mathcal{H}_{\alpha}(C, \eta, M)$ com $M>N_{0}$ então $\widetilde{\mathcal{R}}_{\alpha}^{n}(\phi, t) \in \mathcal{H}_{\alpha}\left(C_{0}, \delta_{0}, M-n\right)$ para $M>n \geq N_{0}$.

Corolário 4.3 Existe $\delta_{0}, C_{0}$ e $N_{0}=N_{0}\left(C_{0}, \delta_{0}\right)$ tal que

- $\widetilde{\mathcal{R}}_{\alpha}^{N_{0}}\left(\mathcal{H}_{\alpha}\left(C_{0}, \delta_{0}, \infty\right)\right) \subset \mathcal{H}_{\alpha}\left(C_{0}, \delta_{0}, \infty\right)$.

- Para todo $C, \eta$ existe $N=N(C, \eta)$ tal que para todo $k \geq 0$ temos

$$
\widetilde{\mathcal{R}}_{\alpha}^{N+k N_{0}}\left(\mathcal{H}_{\alpha}(C, \eta, \infty)\right) \subset \mathcal{H}_{\alpha}\left(C_{0}, \delta_{0}, \infty\right)
$$


Demonstração. Para provar a primeira afirmação do corolário seja $(\phi, t) \in \mathcal{H}_{\alpha}\left(C_{0}, \delta_{0}, \infty\right)$. Pelo teorema anterior existe $N_{0}=N_{0}\left(C_{0}, \delta_{0}\right)$ tal que se $(\phi, t) \in \mathcal{H}_{\alpha}\left(C_{0}, \delta_{0}, \infty\right)$ então $\widetilde{\mathcal{R}}_{\alpha}^{N_{0}}(\phi, t) \in \mathcal{H}_{\alpha}\left(C_{0}, \delta_{0}, \infty\right)$. Assim a primeira afirmação fica provada. Agora pelo Teorema 4.2 para todo $C>0$ e $\eta>0$ existe $N=N(C, \eta)$ tal que se $(\phi, t) \in \mathcal{H}_{\alpha}(C, \eta, \infty)$ então $\widetilde{\mathcal{R}}_{\alpha}^{N}(\phi, t) \in \mathcal{H}_{\alpha}\left(C_{0}, \delta_{0}, \infty\right)$. Logo pela primeira afirmação para todo $k \geq 0$ temos que

$$
\widetilde{\mathcal{R}}_{\alpha}^{N+k N_{0}}(\phi, t) \in \mathcal{H}_{\alpha}\left(C_{0}, \delta_{0}, \infty\right)
$$

Corolário 4.4 Todo ponto fixo $\left(\phi_{\alpha}^{*}, t_{\alpha}^{*}\right)$ unimodal do operador $\widetilde{\mathcal{R}}_{\alpha}$ pertence a $\mathcal{H}_{\alpha}\left(C_{0}, \delta_{0}, \infty\right)$.

Demonstração. Temos que $\left(\phi_{\alpha}^{*}, t_{\alpha}^{*}\right)$ é infinitamente renormalizável com combinatória $\sigma$ isto pois seu correspondente ponto fixo $\phi_{\alpha}^{*} \circ q_{t_{\alpha}^{*}}$ do operador $\mathcal{R}_{\alpha}$ é infinitamente renormalizável com combinatória $\sigma$. Logo $\left(\phi_{\alpha}^{*}, t_{\alpha}^{*}\right) \in \mathcal{H}_{\alpha}(C, \eta, \infty)$, para algum $C>0$ e $\eta>0$. Assim o resultado segue do Teorema 4.2 .

No que segue do presente trabalho vamos a entrar nos detalhes das provas das seguintes afirmacões que motivaram nosso trabalho quando o expoente crítico $\alpha$ é proximo a um número par:

- O operador renormalização $\widetilde{\mathcal{R}}_{\alpha}$ tem um único ponto fixo $\left(\phi_{\alpha}^{*}, t_{\alpha}^{*}\right)$. Além disso o ponto fixo $\left(\phi_{\alpha}^{*}, t_{\alpha}^{*}\right)$ é hiperbólico.

- A variedade estável do ponto fixo $\left(\phi_{\alpha}^{*}, t_{\alpha}^{*}\right)$ tem codimensão um.

- Se o par $(\phi, t)$ é infinitamente renormalizável por $\widetilde{\mathcal{R}}_{\alpha}$. Este faz parte da variedade estável do ponto fixo $\left(\phi_{\alpha}^{*}, t_{\alpha}^{*}\right)$.

Assim começamos definindo um novo operador

$$
F((\phi, t), \alpha)=\widetilde{\mathcal{R}}_{\alpha}(\phi, t)-(\phi, t)
$$

o qual será essencial na prova das duas primeiras afirmacões feitas acima. 
Proposição 4.5 O operador $F$ é analítico complexo nas variáveis $(\phi, t)$ e analítico real na variável $\alpha$.

Demonstração. Isto é conseqüência da Proposição 3.4 .

Teorema 4.6 Existe $\eta>0$ e uma aplicação real analítica $\alpha \rightarrow\left(\phi_{\alpha}^{*}, t_{\alpha}^{*}\right)$, onde $\alpha \in(2 n-$ $\eta, 2 n+\eta)$, tal que $\left(\phi_{\alpha}^{*}, t_{\alpha}^{*}\right)$ é o ponto fixo hiperbólico para o operador $\widetilde{\mathcal{R}}_{\alpha}$, com variedade estável de codimensão um.

Demonstração. Da Proposição 4.5 temos que $F$ é analítico complexo nas variáveis $(\phi, t)$ e analítico real na variável $\alpha$. Além disso

$$
\mathcal{D} \widetilde{\mathcal{R}}_{2 n}\left(\phi_{2 n}^{*}, t_{2 n}^{*}\right)-i d
$$

é invertível porque $\left(\phi_{2 n}^{*}, t_{2 n}^{*}\right)$ é ponto fixo hiperbólico de $\widetilde{\mathcal{R}}_{2 n}$, logo pelo Teorema da Função Implícita existe $\eta>0$ e uma aplicação real analítica $\alpha \rightarrow\left(\phi_{\alpha}^{*}, t_{\alpha}^{*}\right)$, onde $\alpha \in(2 n-\eta, 2 n+\eta)$, tal que

$$
F\left(\left(\phi_{\alpha}^{*}, t_{\alpha}^{*}\right), \alpha\right)=F\left(\left(\phi_{2 n}^{*}, t_{2 n}^{*}\right), 2 n\right)=0
$$

Assim obtemos o ponto fixo hiperbólico $\left(\phi_{\alpha}^{*}, t_{\alpha}^{*}\right)$ para o operador $\widetilde{\mathcal{R}}_{\alpha}$. Note que $\left(\phi_{\alpha}^{*}, t_{\alpha}^{*}\right)$ tem variedade estável de codimensão um.

O seguinte resultado é uma simples consequência do Teorema de Montel e das cotas reais a priori para aplicações infinitamente renormalizáveis, como descrito no Capítulo 1.

Proposição $4.7 S e\left(\phi_{\alpha}, t_{\alpha}\right) \in \mathcal{H}_{\alpha}(C, 2 \eta, \infty)$ e $\alpha \rightarrow \beta$, com $\beta>1$, então existe uma subsequência $\alpha_{i}$ tal que

$$
\left(\phi_{\alpha_{i}}, t_{\alpha_{i}}\right) \rightarrow\left(\phi_{\beta}, t_{\beta}\right)
$$

no espaço $\mathcal{A}_{D_{\eta}} \times \mathbb{C} e\left(\phi_{\beta}, t_{\beta}\right) \in \mathcal{H}_{\beta}(C, \eta, \infty)$.

Teorema 4.8 Para cada $\alpha \sim 2 n$, existe um único ponto fixo unimodal $\left(\phi_{\alpha}^{*}, t_{\alpha}^{*}\right)$ de $\tilde{\mathcal{R}}_{\alpha}, e$ este pertence a $\mathcal{H}_{\alpha}\left(C_{0}, \delta_{0}, \infty\right)$. 
Demonstração. Desde que $\left(\phi_{2 n}^{*}, t_{2 n}^{*}\right)$ é ponto fixo hiperbólico, existe uma vizinhança $V_{1}=$ $B\left(\left(\phi_{2 n}^{*}, t_{2 n}^{*}\right), \eta\right)$ de $\left(\phi_{2 n}^{*}, t_{2 n}^{*}\right)$ tal que para $\alpha \sim 2 n$, existe um único ponto fixo de $\widetilde{\mathcal{R}}_{\alpha}$ em $V_{1}$. Portanto basta mostrarmos que $\alpha \sim 2 n$ todo ponto fixo $\left(\phi_{\alpha}^{*}, t_{\alpha}^{*}\right)$ de $\widetilde{\mathcal{R}}_{\alpha}$ pertence a $V_{1}$. De fato, suponha que existe uma sequência $\alpha_{s} \rightarrow 2 n$, onde $s \rightarrow \infty$, e pontos fixos $\left(\phi_{\alpha_{s}}^{*}, t_{\alpha_{s}}^{*}\right)$ de $\widetilde{\mathcal{R}}_{\alpha_{s}}$ tais que

$$
\left|\phi_{\alpha_{s}}^{*}-\phi_{2 n}^{*}\right|_{D_{\delta}} \geq \eta \text { ou }\left|t_{\alpha_{s}}^{*}-t_{2 n}^{*}\right| \geq \eta
$$

Pelo Corolário 4.3

$$
\left(\phi_{\alpha_{s}}^{*}, t_{\alpha_{s}}^{*}\right) \in \mathcal{H}_{\alpha_{s}}\left(C_{0}, \delta_{0}, \infty\right)
$$

E pela definição de $\widetilde{\widetilde{R}}_{\alpha_{s}}$ temos que

$$
\left(\phi_{\alpha_{s}}^{*}, t_{\alpha_{s}}^{*}\right)=\widetilde{\widetilde{\mathcal{R}}}_{\alpha_{s}}\left(\phi_{\alpha_{s}}^{*}, t_{\alpha_{s}}^{*}\right)
$$

está em $\mathcal{H}_{\alpha_{s}}\left(C_{0}, 2 \delta_{0}, \infty\right)$. Então $\left(\phi_{\alpha_{s}}^{*}, t_{\alpha_{s}}^{*}\right)$ é uma família pré-compacta sobre $\mathcal{A}_{D_{\delta_{0}}} \times \mathbb{C}$, em particular existe uma subsequência $\left(\phi_{\alpha_{s_{i}}}^{*}, t_{\alpha_{s_{i}}}^{*}\right)$ convergindo para $(\phi, t) \in \mathcal{H}_{2 n}\left(C_{0}, \delta_{0}, \infty\right)$. Observe que como

$$
\widetilde{\mathcal{R}}_{\alpha_{s_{i}}}\left(\phi_{\alpha_{s_{i}}}^{*}, t_{\alpha_{s_{i}}}^{*}\right)=\left(\phi_{\alpha_{s_{i}}}^{*}, t_{\alpha_{s_{i}}}^{*}\right),
$$

quando $s_{i} \rightarrow 2 n$, temos que $(\phi, t) \in \mathcal{H}_{2 n}\left(C_{0}, \delta_{0}, \infty\right)$ é um ponto fixo do operador $\widetilde{\mathcal{R}}_{2 n}$. Então pela unicidade do ponto fixo para $\alpha=2 n$ temos

$$
(\phi, t)=\left(\phi_{2 n}^{*}, t_{2 n}^{*}\right)
$$

Isto leva a uma contradição com a Eq. (4.1).

Teorema 4.9 (Convergência) $S e(\phi, t) \in B_{\delta}\left(\left(\phi^{*}, t^{*}\right), \widetilde{\gamma}\right)$ é um par unimodal, infinitamente renormalizável com combinatória $\sigma$, então temos que $\widetilde{\mathcal{R}}_{2 n}^{i}(\phi, t) \in B_{\delta}\left(\left(\phi^{*}, t^{*}\right), \widetilde{\gamma}\right)$, para todo $i$ suficientemente grande $e$

$$
\widetilde{\mathcal{R}}_{2 n}^{i}(\phi, t) \rightarrow_{i}\left(\phi^{*}, t^{*}\right)
$$

em $\mathcal{A}_{D_{\delta}} \times \mathbb{C}$ 
Demonstração. Note que pelas cotas complexas a priori, temos que

$$
\widetilde{\mathcal{R}}_{2 n}^{i}(\phi, t) \in \mathcal{H}_{2 n}\left(C_{0} \cdot \delta_{0}, \infty\right)
$$

para todo $i$ suficientemente grande. Suponhamos que a conclusão do teorema não é verdadeira. Assim existe $\eta>0$ e um par $(\phi, t)$ infinitamente renormalizável com expoente crítico $2 n$ tal que

$$
\left|\widetilde{\mathcal{R}}_{2 n}^{i_{j}}(\phi, t)-\left(\phi^{*}, t^{*}\right)\right|_{\mathcal{A}_{D_{\delta}} \times \mathbb{C}}>\eta
$$

onde $i_{j} \rightarrow_{j} \infty$. Temos que a primeira componente de $\left\{\widetilde{\widetilde{\mathcal{R}}}_{2 n} \circ \widetilde{\mathcal{R}}_{2 n}^{i_{j}-1}(\phi, t)\right\}$ está definida e é univalante em $\bar{D}_{2 \delta}$. Logo esta forma uma família pré-compacta em $\mathcal{A}_{D_{\delta}} \times \mathbb{C}$, em particular existe uma subsequência convergente

$$
\widetilde{\mathcal{R}}_{2 n}^{i_{j}}(\phi, t) \rightarrow(\widetilde{\phi}, \widetilde{t})
$$

Como o operador de composição L satisfaz

$$
L \circ \widetilde{R}_{2 n}=\mathcal{R}_{2 n} \circ L
$$

segue que

$$
\mathcal{R}_{2 n}^{i_{j}}\left(\phi \circ q_{t}\right)=\mathcal{R}_{2 n}^{i_{j}} \circ L(\phi, t) \rightarrow_{j} L(\widetilde{\phi}, \widetilde{t})=\widetilde{\phi} \circ q_{\tilde{t}} .
$$

Por Sullivan [6] (veja também [3]) o operador $\mathcal{R}_{2 n}$ possui um único ponto fixo $\phi^{*} \circ q_{t^{*}}$. Além disso

$$
\mathcal{R}_{2 n}^{i}\left(\phi \circ q_{t}\right) \rightarrow_{i} \phi^{*} \circ q_{t^{*}}
$$

para todo $\phi \circ q_{t}$ infinitamente renormalizável. Assim $\phi^{*} \circ q_{t^{*}}=\widetilde{\phi} \circ q_{\tilde{t}}$. Pela Proposição 2.5 temos que $\widetilde{\phi}=\phi^{*}$ e $\widetilde{t}=t^{*}$. Isto nos leva a uma contradição com a Eq. (4.2).

Na prova do seguinte resultado fazemos uso de aplicações do tipo polinomial, quaseconformalidade, argumento de pullback de Sullivan entre outros. As definições destes podem ser encontrados no apêndice. Para mais detalhes veja [19], [7], [2] e [3]. 
Teorema 4.10 (Equicontinuidade) Seja $(\phi, t) \in \mathcal{H}_{2 n}\left(C, \delta_{0}, \infty\right)$. Então existe $i_{0}$ tal que para todo $\tilde{\gamma}>0$ existe $\tilde{\eta}>0$ com a seguinte propriedade. $\operatorname{Se}(\psi, v) \in \mathcal{H}_{2 n}\left(C, \delta_{0}, \infty\right)$ satisfaz

$$
|(\phi, t)-(\psi, v)|_{\mathcal{A}_{D_{\delta}} \times \mathbb{C}}<\tilde{\eta}
$$

então

$$
\left|\tilde{\mathcal{R}}_{2 n}^{i}(\psi, v)-\tilde{\mathcal{R}}_{2 n}^{i}(\phi, t)\right|_{\mathcal{A}_{D_{\delta}} \times \mathbb{C}}<\tilde{\gamma}
$$

para todo $i \geq i_{0}$.

Demonstração. Primeiramente mostraremos a seguinte afirmação: existe $i_{0}$ tal que para todo $\gamma>0$ existe $\eta>0$ com a seguinte propriedade: Se

$$
(\psi, v) \in \mathcal{H}_{2 n}\left(C, \delta_{0}, \infty\right)
$$

satisfaz

$$
|(\phi, t)-(\psi, v)|_{\mathcal{A}_{D_{\delta_{0}}} \times \mathbb{C}}<\eta
$$

então

$$
\left|\mathcal{R}_{2 n}^{i}\left(\psi \circ q_{v}\right)-\mathcal{R}_{2 n}^{i}\left(\phi \circ q_{t}\right)\right|_{D_{\varepsilon}}<\gamma
$$

para todo $i \geq i_{0}$. De fato, como a aplicação $f:=\phi \circ q_{t}$ é uma aplicação unimodal analítica em uma vizinhança $V_{0}$ do intervalo $[-1,1]$, com ponto crítico de ordem $2 n$, pelo teorema de cotas complexas de Sullivan [6], para $i_{0}$ suficientemente grande existe uma extensão do tipo polinomial

$$
\mathcal{R}_{2 n}^{i_{0}} f: U_{f} \rightarrow U
$$

onde $[-1,1] \subset U_{f} \subset U$. Se $(\psi, v) \in \mathcal{H}_{2 n}\left(C, \delta_{0}, \infty\right)$ é próximo de $(\phi, t)$, então $g=\phi \circ q_{v}$ estará próximo a $f, \log \mathcal{R}_{2 n}^{i_{0}} g$ tem uma extensão do tipo polinomial

$$
\mathcal{R}_{2 n}^{i_{0}} g: U_{g} \rightarrow U
$$

Aqui podemos escolher $U$ de modo que o disco $U_{g}$ move-se holomorficamente com respeito a g. Em particular, via teoria dos movimentos holomórficos, o argumento de pullback 
de Sullivan (veja [19]) e a inexistência de campos de linhas invariantes com suporte no conjunto de Julia cheio de $f$, existe um homeomorfismo quasiconforme $h_{g}: \mathbb{C} \rightarrow \mathbb{C}$ tal que

$$
\left(\mathcal{R}^{i_{0}} g\right) \circ h_{g}(x)=h_{g} \circ\left(\mathcal{R}^{i_{0}} f\right)(x)
$$

para todo $x \in U_{f}$, além disso a quasiconformalidade $Q(g)$ de $h_{g}$ satisfaz

$$
Q\left(\psi \circ q_{v}\right) \rightarrow 1 \text { quando }(\psi, v) \rightarrow(\phi, t)
$$

Note que todas as futuras renormalizações de $f$ e $g$ são conjugadas por reescalonamentos da mesma conjugação $h_{g}$, logo a quasiconformalidade de tais conjugações são igualmente limitadas por $Q\left(\psi \circ q_{v}\right)$. Consequentemente, como $h_{g}$ e todos estes reescalonamentos que dão origem a conjugações entre as renormalizações fixam 1 e -1 , segue que $h_{g}$ e as conjugações entre as correspondentes renormalizações de $f$ e $g$ convergem uniformente para a identidade em partes compactas de $\mathbb{C}$ quando $(\psi, v)$ converge para $(\phi, t)$.

Desde que pelas cotas complexas a priori obtidas no Capítulo 1 , as sequências $\mathcal{R}_{2 n}^{i} f$ e $\mathcal{R}_{2 n}^{i} g$ são limitadas em $\mathcal{U}_{D_{\varepsilon}}$, segue da Eq. (4.3) que se $(\psi, v)$ é suficientemente próximo de $(\phi, t)$ então

$$
\left|\mathcal{R}_{2 n}^{i}\left(\psi \circ q_{v}\right)-\mathcal{R}_{2 n}^{i}\left(\phi \circ q_{t}\right)\right|_{D_{\varepsilon}}<\gamma
$$

se $i \geq i_{0}$. Concluímos portanto a prova da afirmação.

Suponha por absurdo que exista uma sequência $\left(\psi_{j}, v_{j}\right)$ tal que

$$
\left|(\phi, t)-\left(\psi_{j}, v_{j}\right)\right|_{\mathcal{A}_{D_{\delta}} \times \mathbb{C}} \rightarrow{ }_{j} 0
$$

mas

$$
\left|\tilde{\mathcal{R}}_{2 n}^{i_{j}}\left(\psi_{j}, v_{j}\right)-\tilde{\mathcal{R}}_{2 n}^{i_{j}}(\phi, t)\right|_{\mathcal{A}_{D_{\delta}} \times \mathbb{C}}>\tilde{\gamma}
$$

$\operatorname{com} i_{j} \geq i_{0}$ e $i_{j} \rightarrow_{j} \infty$.

Note que, pelas cotas complexas e reais a priori, que

$$
\tilde{\mathcal{R}}_{2 n}^{i_{j}}\left(\psi_{j}, v_{j}\right)=\left(\psi_{j, i_{j}}, v_{j, i_{j}}\right)
$$

onde $\psi_{j, i_{j}}$ é univalente em $D_{2 \delta}$, mantém fixos 1 e -1 e

$$
0<\inf _{j} v_{j, i_{j}} \leq \sup _{j} v_{j, i_{j}}<1
$$


assim nós podemos supor que a segunda coordenada destes pares convergem para algum $v_{\infty} \in(0,1)$. Como $\psi_{i, i_{j}}$ é univalente em $D_{2 \delta}$, tomando uma subsequência, se for necessário, podemos supor que $\psi_{i, i_{j}}$ converge para alguma aplicação univalente $\psi_{\infty}$ em $\bar{D}_{\delta}$. Em particular

$$
\mathcal{R}_{2 n}^{i_{j}}\left(\psi_{j} \circ q_{v_{j}}\right)=L\left(\tilde{\mathcal{R}}_{2 n}^{i_{j}}\left(\psi_{j}, v_{j}\right)\right) \rightarrow_{j} \psi_{\infty} \circ q_{v_{\infty}}
$$

Por outro lado, pela afirmação que provamos no início desta demonstração e o Teorema 4.9 obtemos

$$
\mathcal{R}_{2 n}^{i_{j}}\left(\psi_{j} \circ q_{v_{j}}\right) \rightarrow_{j} \phi^{*} \circ q_{t^{*}}
$$

Logo $\psi_{\infty} \circ q_{v_{\infty}}=\phi^{*} \circ q_{t^{*}}$. Pela Proposição 2.5 concluímos que

$$
\left(\psi_{\infty}, v_{\infty}\right)=\left(\phi^{*}, t^{*}\right)
$$

Assim

$$
\left|\tilde{\mathcal{R}}_{2 n}^{i_{j}}\left(\psi_{j}, v_{j}\right)-\left(\phi^{*}, t^{*}\right)\right|_{\mathcal{A}_{D_{\delta}} \times \mathbb{C}} \rightarrow_{j} 0 .
$$

Mas Eq. (4.4) e o Teorema 4.9 implicam que

$$
\left|\tilde{\mathcal{R}}_{2 n}^{i_{j}}\left(\psi_{j}, v_{j}\right)-\left(\phi^{*}, t^{*}\right)\right|_{\mathcal{A}_{D_{\delta}} \times \mathbb{C}}>\tilde{\gamma} / 2
$$

para $j$ suficientemente grande, o que certamente contradiz Eq. (4.5).

Seja $a>0$ uma constante. Denotemos por $B_{a}^{\infty}\left(\phi_{\alpha}^{*}, t_{\alpha}^{*}\right)$ o conjunto de pares de aplicações infinitamente renormalizável $(\phi, t)$ por $\widetilde{\mathcal{R}}_{\alpha}$ tal que

$$
\left|(\phi, t)-\left(\phi_{\alpha}^{*}, t_{\alpha}^{*}\right)\right|_{\mathcal{A}_{D_{\delta}} \times \mathbb{C}}<a .
$$

Teorema 4.11 Para todo $\gamma>0$ existe $N_{2}$ tal que para todo $\alpha \sim 2 n$ temos

$$
\widetilde{\mathcal{R}}_{\alpha}^{N_{2}}\left(\mathcal{H}_{\alpha}\left(C_{0}, \delta_{0}, \infty\right)\right) \subset B_{\gamma}^{\infty}\left(\phi_{2 n}^{*}, t_{2 n}^{*}\right)
$$

Demonstração. Seja $\gamma>0$. Afirmamos que existe $M_{2}$ tal que

$$
\widetilde{\mathcal{R}}_{2 n}^{M_{2}}\left(\mathcal{H}_{2 n}\left(C_{0}, \delta_{0}, \infty\right)\right) \subset B_{\gamma / 2}^{\infty}\left(\phi_{2 n}^{*}, t_{2 n}^{*}\right)
$$


De fato, suponhamos que existe uma sequência $\left(\phi_{i}, t_{i}\right) \in \mathcal{H}_{2 n}\left(C_{0}, \delta_{0}, \infty\right)$ tal que para uma subsequência $j_{i} \rightarrow \infty$ temos que

$$
\left|\widetilde{\mathcal{R}}_{2 n}^{j_{i}}\left(\phi_{i}, t_{i}\right)-\left(\phi_{2 n}^{*}, t_{2 n}^{*}\right)\right|_{\mathcal{A}_{D_{\delta}} \times \mathbb{C}}>\gamma / 2
$$

Pelo Corolário 4.3 obtemos que $\widetilde{\widetilde{\mathcal{R}}}_{2 n}\left(\phi_{i}, t_{i}\right) \in \mathcal{H}_{2 n}\left(C_{0}, 2 \delta_{0}, \infty\right)$, em particular temos que forma uma família pré-compacta em $\mathcal{A}_{D_{\delta_{0}}} \times \mathbb{C}$. Assim existe uma subsequência, a qual denotamos da mesma forma, tal que

$$
\widetilde{\mathcal{R}}_{2 n}\left(\phi_{i}, t_{i}\right)=j \circ \widetilde{\widetilde{\mathcal{R}}}_{2 n}\left(\phi_{i}, t_{i}\right) \rightarrow(\phi, t),
$$

onde $(\phi, t) \in \mathcal{H}_{2 n}\left(C_{0}, \delta_{0}, \infty\right)$. Pelo Teorema 4.9 temos que para $\gamma>0$ existe $k_{0}>0$ tal que para todo $k>k_{0}$

$$
\left|\widetilde{\mathcal{R}}_{2 n}^{k}\left(\phi_{i}, t_{i}\right)-\left(\phi_{2 n}^{*}, t_{2 n}^{*}\right)\right|_{\mathcal{A}_{D_{\delta}} \times \mathbb{C}}<\frac{\gamma}{8}
$$

E fazendo uso do Teorema 4.10 existe $i_{0}>0$ e $k_{1}>0$ tal que para todo $i>i_{0}$ e $k>k_{1}$ temos que

$$
\left|\widetilde{\mathcal{R}}_{2 n}^{k}\left(\widetilde{\mathcal{R}}_{2 n}\left(\phi_{i}, t_{i}\right)\right)-\widetilde{\mathcal{R}}_{2 n}^{k}(\phi, t)\right|_{\mathcal{A}_{D_{\delta}} \times \mathbb{C}}<\frac{\gamma}{8} .
$$

Logo para $k>\max \left\{k_{0}, k_{1}\right\}$ obtemos

$$
\left|\widetilde{\mathcal{R}}_{2 n}^{k+1}\left(\phi_{i}, t_{i}\right)-\left(\phi_{2 n}^{*}, t_{2 n}^{*}\right)\right|_{\mathcal{A}_{D_{\delta}} \times \mathbb{C}}<\frac{\gamma}{4}
$$

o que contradiz a Eq. (4.8). Logo a afirmação acima é verdadeira.

Afirmamos que podemos tomar $N_{2}=M_{2}+1$ para $\alpha \sim 2 n$. Caso contrário existe uma sequência $\alpha_{s} \rightarrow 2 n$ e $\left(\phi_{s}, t_{s}\right) \in \mathcal{H}_{\alpha_{s}}\left(C_{0}, \delta_{0}, \infty\right)$ tal que

$$
\left|\widetilde{\mathcal{R}}_{\alpha_{s}}^{M_{2}+1}\left(\phi_{s}, t_{s}\right)-\left(\phi_{2 n}^{*}, t_{2 n}^{*}\right)\right|_{\mathcal{A}_{D_{\delta}} \times \mathbb{C}}>\gamma
$$

Temos que primeira componente dos pares na família $\left\{\widetilde{\widetilde{\mathcal{R}}}_{\alpha_{s}}\left(\phi_{s}, t_{s}\right)\right\}$ tem uma extensão complexa univalente para $D_{2 \delta_{0}}$. Assim esta é uma família pré-compacta sobre $\mathcal{A}_{D_{\delta_{0}}} \times \mathbb{C}$. Em particular existe uma subsequência $\widetilde{\widetilde{\mathcal{R}}}_{\alpha_{s_{i}}}\left(\phi_{s_{i}}, t_{s_{i}}\right)$ sobre $\mathcal{A}_{D_{\delta_{0}}} \times \mathbb{C}$ que converge para $(\widetilde{\phi}, \widetilde{t}) \in \mathcal{H}_{2 n}\left(C_{0}, \delta_{0}, \infty\right)$. Da afirmação acima temos

$$
\widetilde{\mathcal{R}}_{2 n}^{M_{2}}(\widetilde{\phi}, \widetilde{t}) \in B_{\gamma / 2}^{\infty}\left(\phi_{2 n}^{*}, t_{2 n}^{*}\right) .
$$


Por outro lado

$$
\widetilde{\mathcal{R}}_{\alpha_{s_{i}}}^{M_{2}+1}\left(\phi_{s_{i}}, t_{s_{i}}\right) \rightarrow \widetilde{\mathcal{R}}_{2 n}^{M_{2}}(\widetilde{\phi}, \widetilde{t})
$$

em $\mathcal{A}_{D_{\delta_{0}}} \times \mathbb{C}$. Assim para $i$ suficientemente grande temos

$$
\left|\widetilde{\mathcal{R}}_{\alpha_{s_{i}}}^{M_{2}+1}\left(\phi_{s_{i}}, t_{s_{i}}\right)-\left(\phi_{2 n}^{*}, t_{2 n}^{*}\right)\right|_{\mathcal{A}_{D_{\delta}} \times \mathbb{C}}<\gamma,
$$

o que contradiz Eq. (4.9).

Definição 4.12 A variedade estável do ponto fixo $\left(\phi_{\alpha}^{*}, t_{\alpha}^{*}\right)$, denotado por $W^{s}=W^{s}\left(\phi_{\alpha}^{*}, t_{\alpha}^{*}\right)$, é definida como

$$
W^{s}:=\left\{(\phi, t) \text { unimodal }: \widetilde{\mathcal{R}}_{\alpha}^{n}(\phi, t) \rightarrow_{n}\left(\phi_{\alpha}^{*}, t_{\alpha}^{*}\right)\right\}
$$

Por

$$
\widetilde{\mathcal{R}}_{\alpha}^{n}(\phi, t) \rightarrow_{n}\left(\phi_{\alpha}^{*}, t_{\alpha}^{*}\right)
$$

entende-se que $\widetilde{\mathcal{R}}_{\alpha}^{n}(\phi, t)$ pertence a $\mathcal{A}_{D_{\delta}} \times \mathbb{C}$ para $n$ suficientemente grande, e que $\widetilde{\mathcal{R}}_{\alpha}^{n}(\phi, t)$ converge para $\left(\phi_{\alpha}^{*}, t_{\alpha}^{*}\right)$ em $\mathcal{A}_{D_{\delta}} \times \mathbb{C}$.

Definição 4.13 Seja $V_{1}$ uma vizinhança do ponto fixo $\left(\phi_{\alpha}^{*}, t_{\alpha}^{*}\right)$ do operador $\widetilde{\mathcal{R}}_{\alpha}$. Defina

$$
\mathcal{N}_{V_{1}}^{k}\left(\phi_{\alpha}^{*}, t_{\alpha}^{*}\right):=\left\{(\phi, t): \widetilde{\mathcal{R}}_{\alpha}^{j}(\phi, t) \in V_{1}, 0 \leq j \leq k\right\}
$$

onde $k \in \mathbb{N} \cup \infty$. Além disso, definimos

$$
\mathcal{N}_{V_{1}}^{\infty}\left(\phi_{\alpha}^{*}, t_{\alpha}^{*}\right):=\bigcap_{k \in \mathbb{N}} \mathcal{N}_{V_{1}}^{k}\left(\phi_{\alpha}^{*}, t_{\alpha}^{*}\right),
$$

ou seja, o conjunto dos pares tal que todos os seus iterados estão próximos de $\left(\phi_{\alpha}^{*}, t_{\alpha}^{*}\right)$.

Agora estamos prontos para definir a variedade estável local.

Definição 4.14 Para cada vizinhança $V$ de $\left(\phi_{\alpha}^{*}, t_{\alpha}^{*}\right)$ definimos a correspondente variedade estável local

$$
W_{V}^{s}\left(\phi_{\alpha}^{*}, t_{\alpha}^{*}\right):=W^{s}\left(\phi_{\alpha}^{*}, t_{\alpha}^{*}\right) \cap \mathcal{N}_{V}^{\infty}\left(\phi_{\alpha}^{*}, t_{\alpha}^{*}\right) .
$$

Como $\left(\phi_{\alpha}^{*}, t_{\alpha}^{*}\right)$ é ponto fixo hiperbólico, podemos escolher $V_{1}$ convenientemente de modo que

$$
W_{V_{1}}^{s}\left(\phi_{\alpha}^{*}, t_{\alpha}^{*}\right):=\mathcal{N}_{V_{1}}^{\infty}\left(\phi_{\alpha}^{*}, t_{\alpha}^{*}\right)
$$


Teorema 4.15 Para $\alpha \sim 2 n$ temos que todo par unimodal $(\phi, t)$, infinitamente renormalizável com combinatória $\sigma$ e com ordem $\alpha$, pertence a variedade estável do único ponto fixo unimodal $\left(\phi_{\alpha}^{*}, t_{\alpha}^{*}\right)$ de $\tilde{\mathcal{R}}_{\alpha}$, isto é

$$
\bigcup_{C>0} \bigcup_{\eta>0} \mathcal{H}_{\alpha}(C, \eta, \infty) \subseteq W^{s}\left(\phi_{\alpha}^{*}, t_{\alpha}^{*}\right)
$$

Demonstração. Seja $V_{1}$ satisfazendo a Eq. (4.10). Escolha $\gamma>0$ tal que $B_{\gamma}\left(\phi_{2 n}^{*}, t_{2 n}^{*}\right)$ está contido em $V_{1}$.

Afirmação I. Existe $\gamma_{1}$ tal que para todo $\alpha \sim 2 n$ temos

$$
B_{\gamma_{1}}^{\infty}\left(\phi_{2 n}^{*}, t_{2 n}^{*}\right) \subset W_{V_{1}}^{s}
$$

De fato, seja $N_{2}$ como no Teorema 4.11. Escolha $\gamma_{1}<\gamma$ suficientemente pequeno tal que para todo $i=1, . ., N_{2}$,

$$
\widetilde{\mathcal{R}}_{\alpha}^{i} B_{\gamma_{1}}^{\infty}\left(\phi_{2 n}^{*}, t_{2 n}^{*}\right)=\widetilde{\widetilde{\mathcal{R}}}_{\alpha} \circ \widetilde{\mathcal{R}}_{\alpha}^{i-1} B_{\gamma_{1}}^{\infty}\left(\phi_{2 n}^{*}, t_{2 n}^{*}\right) \subset B_{\gamma}^{\infty}\left(\phi_{2 n}^{*}, t_{2 n}^{*}\right) \cap \mathcal{H}_{\alpha}\left(C_{0}, \delta_{0}, \infty\right) .
$$

Pelo Teorema 4.11 obtemos $\widetilde{\mathcal{R}}_{\alpha}^{i+k N_{2}} B_{\gamma_{1}}^{\infty}\left(\phi_{2 n}^{*}, t_{2 n}^{*}\right) \subset B_{\gamma}^{\infty}\left(\phi_{2}^{*}, t_{2}^{*}\right) \subset V_{1}$, para todo $k$ e $i=$ $1, . ., N_{2}$. Assim segue a afirmação.

Afirmação II. Se $(\phi, t) \in \mathcal{H}_{\alpha}(C, \eta, \infty)$, para algum $C>0$ e $\eta>0$, existe $N>0$ tal que

$$
\widetilde{\mathcal{R}}_{\alpha}^{N}(\phi, t) \in B_{\gamma_{1}}^{\infty}\left(\phi_{2 n}^{*}, t_{2 n}^{*}\right)
$$

no espaço $\mathcal{A}_{D_{\delta_{0}}} \times \mathbb{C}$. De fato, pelo Teorema 4.2 existe $N_{0}$ tal que para $\alpha \sim 2 n$ e $(\phi, t) \in$ $\mathcal{H}_{\alpha}(C, \eta, \infty)$,

$$
\widetilde{\mathcal{R}}_{\alpha}^{N_{0}}(\phi, t) \in \mathcal{H}_{\alpha}\left(C_{0}, \delta_{0}, \infty\right) .
$$

E pelo Teorema 4.11 para todo $\gamma_{1}>0$ existe $N_{2}$ tal que

$$
\widetilde{\mathcal{R}}_{\alpha}^{N_{0}+N_{2}}(\phi, t) \in B_{\gamma_{1}}^{\infty}\left(\phi_{2 n}^{*}, t_{2 n}^{*}\right) .
$$

Assim usando as afirmações acima segue facilmente que

$$
\bigcup_{C>0} \bigcup_{\eta>0} \mathcal{H}_{\alpha}(C, \eta, \infty) \subseteq W^{s}\left(\phi_{\alpha}^{*}, t_{\alpha}^{*}\right) .
$$




\section{Apêndice A}

\section{Operadores compactos}

\section{A.1 Espectro de um operador compacto}

Sejam $E, F$ espaços de Banach. Denote $\mathcal{L}(E, F)$ o espaço de operadores lineares com domínio $E$ e posto em $F$.

Definição A.1 Dizemos que $T \in \mathcal{L}(E, F)$ é compacto se, para cada sequência limitada $\left\{x_{n}\right\}$ em E, a seqüencia $\left\{T x_{n}\right\}$ contem uma subsequência convergindo para algum limite em $F$. Denote por $\mathcal{K}(E, F)$ o conjunto de operadores compactos e defina $\mathcal{K}(E)=\mathcal{K}(E, E)$.

Observe que um operador linear compacto é contínuo. Pois, a descontinuidade de $T$ poderia implicar a existência de uma sequência $\left\{x_{n}\right\}$ tal que $\left|x_{n}\right| \leq 1$ e $\left|T x_{n}\right| \rightarrow \infty$, e isto não pode acontecer se $T$ é compacto.

Definição A.2 Seja $T \in \mathcal{L}(E)$. O conjunto resolvente é

$$
\rho(T)=\{\lambda \in \mathbb{C}:(T-\lambda I) \in \mathcal{L}(E) \text { é bijetivo }\} .
$$


$O$ espectro $\sigma(T)$ é o complemento do conjunto resolvente, $\sigma(T)=\mathbb{R} \backslash \rho(T)$. Dizemos que $\lambda$ é autovalor e escrevemos $\lambda \in V P(T)$ se

$$
N(T-\lambda I) \neq 0
$$

Teorema A.3 Seja $T \in \mathcal{K}(E)$, com $\operatorname{dim} E=\infty$. Então

1. $0 \in \sigma(T)$,

2. $\sigma(T) \backslash\{0\}=V P(T) \backslash\{0\}$,

3. temos uma das seguintes proposições

- $\sigma(T)=0$

- $\sigma(T) \backslash\{0\}$ é finito,

- $\sigma(T) \backslash\{0\}$ é uma sequência acumulando em zero.

Teorema A.4 Se $T \in \mathcal{K}(E)$, com $\operatorname{dim} E=\infty$, e $\lambda$ é um escalar não nulo, então o autoespaço associado a $\lambda, N(T-\lambda I)$, é finito-dimensional. 


\section{Apêndice B}

\section{Resultados de análise complexa}

\section{B.1 Aplicacões holomorfas}

Nós fazemos uso do seguinte resultado na extensão dos operadores de renormalização para operadores analíticos complexos.

Lema B.1 Sejam $f_{i}: \bar{V}_{i} \rightarrow \mathbb{C}, 1 \leq i \leq n$, aplicações contínuas, holomorfas no domínio $V_{i}$ respectivamente e $K$ um conjunto compacto contido em $V_{1}$ tal que $f_{i} \circ f_{i-1} \circ \cdots \circ f_{1}$ esta definido em $K$, e $f_{i} \circ f_{i-1} \circ \cdots \circ f_{1}(K) \subset V_{i+1}$, para todo $i<n$. Então existe $\gamma=\gamma(K)$ tal que se $g_{i}: \bar{V}_{i} \rightarrow \mathbb{C}$ são funções holomorfas que satisfazem

$$
\left|g_{i}-f_{i}\right|_{V_{i}} \leq \gamma
$$

então $g_{i} \circ g_{i-1} \circ \cdots \circ g_{1}$ está definido em $K$, para todo $i \leq n$.

Outra das ferramentas importantes usada ao longo do trabalho foi o Teorema de Montel.

Definição B.2 Seja X uma variedade complexa, e seja $\mathcal{F}$ uma família de aplicações holomorfas $f: X \rightarrow \hat{\mathbb{C}}$. Então $\mathcal{F}$ é uma família normal se para cada sequência $f_{n}$ em $\mathcal{F}$ 
tem uma subsequência a qual converge uniformemente sobre subconjuntos compactos de $X$. O limite $f_{\infty}$ é de novo uma aplicação holomorfa com valores em $\hat{\mathbb{C}}$.

Teorema B.3 (Montel) Seja X uma variedade complexa, e seja $\mathcal{F}$ uma familia de aplicações holomorfas $f: X \rightarrow \widehat{\mathbb{C}}$ as quais omitem três valores. Isto é, existem três pontos distintos $a, b, c \in \hat{\mathbb{C}}$ tal que $f(X) \subset \hat{\mathbb{C}} \backslash\{a, b, c\}$ para qualquer $f \in \mathcal{F}$. Então $\mathcal{F}$ é uma família normal.

A prova deste resultado segue do Lema de Schwarz e o fato que a esfera de três buracos é coberta pelo disco unitário. Mais generalmente temos

Corolário B.4 Seja $s_{i}: X \rightarrow \hat{\mathbb{C}}, i=1,2,3$ três aplicações holomorfas cujos gráficos são disjuntos. Então o conjunto $\mathcal{F}$ de aplicações holomorfas $f: X \rightarrow \hat{\mathbb{C}}$ cujos gráficos são disjuntos dos gráficos de $\left\{s_{1}, s_{2}, s_{3}\right\}$ é uma família normal.

\section{B.2 Aplicações quaseconformes}

Qualquer superfície de Riemann $A$ com $\pi_{1}(A) \cong \mathbb{Z}$ é isomorfo a $\mathbb{C} \backslash\{0\}$, disco unitário menos o zero ou o anel

$$
A(R)=\{z: 1<|z|<R\}
$$

para algum $R \in(1, \infty)$. No caso que $A$ é isomorfo a $A(R)$, o modulo de $A$ é definido por

$$
\bmod (A)=\frac{\log R}{2 \pi}
$$

Por convenção $\bmod (A)=\infty$ nos outros dois casos.

Definição B.5 Um homeomorfismo $f: X \rightarrow Y$ entre superficies de Riemann $X$ e $Y$ é $K$-quaseconforme, $K \geq 1$ se para todo anel $A \subset X$,

$$
\frac{1}{K} \bmod (A) \leq \bmod f(A) \leq K \bmod (A) \text {. }
$$

Uma aplicação $f$ é 1-quaseconforme se e somente se $f$ é conforme no sentido usual. 


\section{B.3 Aplicações do tipo polinomial}

Para qualquer polinômio $f: \mathbb{C} \rightarrow \mathbb{C}$ de grau $d>1$, o conjunto de Julia cheio $K(f)$ é o conjunto compacto de pontos cujas iterações permanecem limitadas; sua fronteira é o conjunto de Julia $J(f)$.

Definição B.6 Uma aplicação do tipo polinomial $f: U \rightarrow V$ é uma aplicação holomorfa própria entre domínios simplesmente conexos $U$ e $V$ em $\mathbb{C}$ tal que $\bar{U}$ é um subconjunto compacto de $V$.

O conjunto de Julia cheio $K(f)$ é definido por

$$
K(f)=\bigcap_{1}^{\infty} f^{n}(U) ;
$$

e o conjunto de Julia $J(f)$ é igual à fronteira de $K(f)$ em $\mathbb{C}$.

Definição B.7 Sejam $f$ e $g$ duas aplicações do tipo polinomial. Dizemos que $f$ e $g$ são equivalentes híbridos se existe uma conjugação quaseconforme $\phi$ entre elas, definida numa vizinhança dos seus respetivos conjuntos de Julia cheio, tal que $\bar{\partial} \phi=0$ sobre $K(f)$.

O seguinte resultado é devido a Doudy e Hubbard:

Teorema B.8 Qualquer aplicação do tipo polinomial $f$ é equivalente híbrido a (uma restrição apropriada de) um polinômio $g$ do mesmo grau. Quando $K(f)$ é conexo, o polinômio g é único a menos de uma conjugação afim. 



\section{Referências Bibliográficas}

[1] A. Avila, M. Martens, And W.de Melo, On the dynamics of the renormalization operator, Global Analysis On Dynamical Systems, p. 449-460, (2001).

[2] C. McMullen, Complex dynamics and renormalization, Annals of Mathematics Studies,135, Princeton University Press,Princeton, (1994).

[3] C. McMullen, Renormalization and 3-manifolds which fiber over the circle, Annals of Mathematics Studies 142, Princeton University Press, Princeton, (1996).

[4] D. Smania, Complex bounds for multimodal maps: bounded combinatorics, Institute of Physics Publising, Nonlinearity 14 1311-1330, (2001).

[5] D. Smania, On the hyperbolicity of the period-doubling fixed point, Transactions of the American Mathematical Society, ISSN 0002-9947, Vol. 358, No 4, p. 1827-1846, (2006).

[6] D. Sullivan, Bounds, quadratic diferentials and renormalization conjectures, AMS Centennial Publications 2, Mathematics into the Twenty-first Century, A.M.S., Providence, RI, p. 417-466, (1992).

[7] E.de FARIA And W.de Melo, One dimensional dynamics: the mathematical tools, Publicações do 23o Colóquio Brasileiro de Matemática (2001).

[8] E.de Faria, W.de Melo, And A. Pinto, Global hyperbolicity of renormalization for $C^{r}$ unimodal mappings, Ann. of Math., Princeton, v. 164, p. 731-824, (2006). 
[9] G. Bachman and L. Narici, Functional analysis, Brooklyn: New York, Academic Press International Edition (1966).

[10] G. Levin And S. VAn Strien, Local connectivity of the Julia set of real polynomials, Ann. of Math. (2) 147, no. 3, p. 471-541 (1998).

[11] J. Graczyk and G. Swiatek, Polynomial-like property for real quadratic polynomials, Topology Proc. 21, p. 33-112 (1996).

[12] John B. Conway, Functions of one complex variable I, Springer-Verlag, 2nd ed. (1978).

[13] John B. Conway, Functions of one complex variable II, Springer-Verlag, (1995).

[14] M. LyUbich, Dynamics of quadratic polynomials, I,II Acta Math. 178 185-297, (1997).

[15] M. Lyubich And M. Yampolsky, Dynamics of quadratic polynomials:complex bounds for real maps, Ann. Inst. Fourier (Grenoble) 47 1249-55, (1997).

[16] M. Lyubich, Feigenbaum-Coullet-Tresser universality and Milnor's Hairiness Conjecture, Ann. of Math.(2), 149 319-420, (1999).

[17] M. Martens, The periodic points of renormalization, Ann. of Math. 147, p. 543-584, (1998).

[18] Serge Lang, Differential manifolds, Addison-Wesley Publishing Company, (1972).

[19] W. De Melo and S. van Strien, One-dimensional dynamics, 1. ed. SpringerVerlag, (1993).

[20] W. Rudin, Real and Complex Analysis, McGraw-Hill Book Co., New York, (1987). 


\section{Índice Remissivo}

Aplicação

do tipo polinomial, 69

renormalizável, 12

unimodal, 9

univalente, 16

Autoespaço, 52, 66

Autovalor, 50, 66

Ciclo, 10

Combinatória limitada, 14

Conjunto

$2 n$-simétrico, 34

a-estádio, 18, 36

de Julia cheio, 69

Cota

complexa a priori, 20

real a priori, 22

Espectro, 66

Família normal, 67

Operador

compacto, 65

composição, 37

de renormalização $\widetilde{\mathcal{R}}, 14,44$ de renormalização $\mathcal{R}, 12,43$

Par

renormalizável, 12

unimodal, 22

Período da renormalização, 12

Permutação, 10

Permutação unimodal, 10

Quaseconforme, 68

Teorema

de Montel, 68

Aproximação polinomial, 34

Complex Bounds, 21

de Distorção de Koebe, 16

Generalizado de Distorção, 18

Variedade estável, 63 